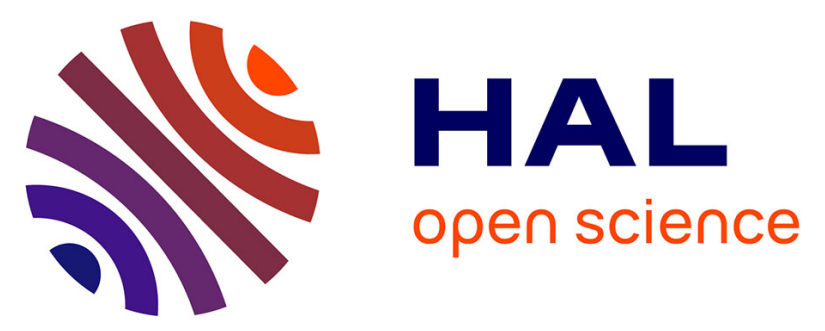

\title{
Flow past a cylinder with a flexible splitter plate: A complementary experimental-numerical investigation and a new FSI test case (FSI-PfS-1a)
}

Guillaume de Nayer, Andreas Kalmbach, Michael Breuer, Stefan Sicklinger, Roland Wüchner

\section{To cite this version:}

Guillaume de Nayer, Andreas Kalmbach, Michael Breuer, Stefan Sicklinger, Roland Wüchner. Flow past a cylinder with a flexible splitter plate: A complementary experimental-numerical investigation and a new FSI test case (FSI-PfS-1a). Computers and Fluids, 2014, 99, pp.18-43. 10.1016/j.compfluid.2014.04.020 . hal-01018342

\section{HAL Id: hal-01018342 \\ https://hal.science/hal-01018342}

Submitted on 4 Jul 2014

HAL is a multi-disciplinary open access archive for the deposit and dissemination of scientific research documents, whether they are published or not. The documents may come from teaching and research institutions in France or abroad, or from public or private research centers.
L'archive ouverte pluridisciplinaire HAL, est destinée au dépôt et à la diffusion de documents scientifiques de niveau recherche, publiés ou non, émanant des établissements d'enseignement et de recherche français ou étrangers, des laboratoires publics ou privés.

\section{(ㅇ)(1) $\$$}

Distributed under a Creative Commons Attribution - NonCommercial - NoDerivatives 44.0 


\title{
Flow past a cylinder with a flexible splitter plate: a complementary experimental-numerical investigation and a new FSI test case (FSI-PfS-1a)
}

\author{
G. De Nayer ${ }^{\mathrm{a}}$, A. Kalmbach ${ }^{\mathrm{a}}$, M. Breuer ${ }^{\mathrm{a}, *}$, S. Sicklinger ${ }^{\mathrm{b}}$, R. Wüchner ${ }^{\mathrm{b}}$ \\ ${ }^{a}$ Professur für Strömungsmechanik, Helmut-Schmidt-Universität Hamburg, D-22043 Hamburg, Germany \\ ${ }^{b}$ Lehrstuhl für Statik, Technische Universität München, D-80290 München, Germany
}

\begin{abstract}
The objective of the present paper is to provide a challenging and well-defined validation test case for fluid-structure interaction (FSI) in turbulent flow to close a gap in the literature. The following list of requirements are taken into account during the definition and setup phase. First, the test case should be geometrically simple which is realized by a classical cylinder flow configuration extended by a flexible structure attached to the backside of the cylinder. Second, clearly defined operating and boundary conditions are a must and put into practice by a constant inflow velocity and channel walls. The latter are also evaluated against a periodic setup relying on a subset of the computational domain. Third, the material model should be widely used. Although a rubber plate is chosen as the flexible structure, it is demonstrated by additional structural tests that a classical St. Venant-Kirchhoff material model is sufficient to describe the material behavior appropriately. Fourth, the flow should be in the turbulent regime. Choosing water as the working fluid and a medium-size water channel, the resulting Reynolds number of $\mathrm{Re}=30,470$ guarantees a sub-critical cylinder flow with transition taking place in the separated shear layers. Fifth, the test case results should be underpinned by a detailed validation process. For this purpose complementary numerical and experimental investigations were carried out. Based on optical contactless measuring techniques (particle-image velocimetry and laser distance sensor) the phase-averaged flow field and the structural deformations were determined. These data were compared with corresponding numerical predictions relying on large-eddy simulations and a recently developed semi-implicit predictor-corrector FSI coupling scheme. Both results were found to be in close agreement showing a quasi-periodic oscillating flexible structure in the first swiveling FSI mode with a corresponding Strouhal number of about $\mathrm{St}_{\mathrm{FSI}}=0.11$.
\end{abstract}

Keywords: Fluid-structure interaction (FSI); FSI validation test case; FSI benchmark; turbulent reference experiment; particle-image velocimetry (PIV); coupled numerical simulation; large-eddy simulation (LES); shell.

\section{Introduction}

A flexible structure exposed to a fluid flow is deformed and deflected owing to the fluid forces acting on its surface. These displacements influence the flow field resulting in a coupling process between the fluid and the structure shortly denoted fluid-structure interaction (FSI).

${ }^{*}$ Corresponding author

Email address: breuer@hsu-hh.de (M. Breuer) 
Due to its manifold forms of appearance it is a topic of major interest in many fields of engineering. Based on enhanced numerical algorithms and increased computational resources numerical simulations have become an important and valuable tool for solving this kind of problem within the last decade. Today FSI simulations complement additional experimental investigations. A long-lasting vision of the computational engineer is to completely replace or at least strongly reduce expensive experimental investigations in the foreseeable future. However, to attain this goal validated and thus reliable simulation tools are required.

The long-term objective of the present research project is the coupled simulation of big lightweight structures such as thin membranes exposed to turbulent flows (outdoor tents, awnings...). To study these complex FSI problems, a multi-physics code framework was recently developed (Breuer et al., 2012). In order to assure reliable numerical simulations of complex configurations, the whole FSI code needs to be validated at first on simpler test cases with trusted reference data. In Breuer et al. (2012) the verification process of the code developed is detailed. The computational fluid dynamics (CFD) and computational structural dynamics (CSD) solvers were at first checked separately and then, the coupling algorithm was considered in detail based on a laminar benchmark. A 3D turbulent example was also taken into account to prove the applicability of the newly developed coupling scheme in the context of large-eddy simulations (LES). However, owing to missing reference data a full validation was not possible. The overall goal of the present paper is to present a turbulent FSI test case supported by experimental data and numerical predictions based on the multi-physics code developed. Thus, on the one hand the current FSI methodology involving LES and shell structures undergoing large deformations is validated. On the other hand, a new turbulent FSI validation test case is defined based on detailed measurements and with specific insights into numerical flow simulations, computational structural analysis as well as coupling issues. Hence, the present study should provide a precisely described test case to the FSI community for the technically relevant case of turbulent flows interacting with flexible thin structures. To propose a new FSI test case supported by experimental data a brief literature study of the available FSI test cases with simple flexible thin structures has to be done. These validation test cases can be divided into two groups: the laminar and the turbulent cases. For the sake of brevity complicated FSI cases are ignored in the following summary.

As laminar, purely numerical FSI test cases one can cite the 2D and 3D modified cavity flows of Wall (1999) and Mok (2001), taken as example in Förster et al. (2007): This is a modification of the well-known lid-driven cavity CFD benchmark with a flexible membrane at the bottom. The CFD part of the FSI code can be validated at first with the classical lid-driven cavity flow. Then based on a simple modification assuming a flexible instead of a rigid bottom wall, the FSI coupling algorithm can be evaluated. This test is purely numerical and no experimental data are provided.

From the very first, the hemodynamics research domain was interested in FSI to study blood flow in flexible veins and arteries. Therefore, as 2D and 3D numerical laminar test cases the model of a compliant vessel of Nobile (2001) and Formaggia et al. (2001) have to be cited. This unsteady test case is often used to validate FSI codes relying on shells, because of its simplicity and of the 3D structure deformations. Regarding other laminar benchmarks, there are many FSI test cases with elastic plates: a very simple test case is the 2D numerical laminar test case used by Glück et al. (2001). A cantilever plate is transversely put into a flow. The solution is stationary and the displacement is small. It is too simple to validate a FSI code, but very useful to debug and evaluate the coupling scheme. In Glück et al. (2001) another test case is presented: a L-shaped flexible body is located in a laminar flow and mounted headlong at the bottom wall. This case is 3D and stationary, at least for moderate Reynolds numbers. It 
is useful for first 3D coupling tests, but no experimental data are provided. Balint and Lucey (2005) carried out a 2D cantilever plate in axial flow in order to describe human snoring caused by flutter of the soft palate. Two Reynolds numbers are tested with large deflections of the plate and numerical flow results are provided. More complicated is the 2D numerical laminar benchmark of Wall and Ramm (1998), which was later modified by Hübner et al. (2004): A thin elastic cantilever plate is attached behind a rigid square cylinder. The geometry is simple, but the deformations of the structure are significant, which implies a good structure model for the great displacements expected and an appropriate remeshing or robust mesh moving procedure for the CFD solver. Moreover, the artificial added-mass effect is strong. Therefore, it represents an appropriate benchmark to test the coupling method (Boyer et al., 2011).

The well-known 2D numerical laminar benchmarks of Turek and Hron (2006) and Turek et al. (2010) developed in a collaborative research effort on FSI (Bungartz et al., 2010) have to be cited here, too: An elastic cantilever plate is clamped behind a rigid circular cylinder. Three different test cases, named FSI1, FSI2 and FSI3 are provided, complemented by additional selfcontained CFD and CSD test cases to check both solvers independently. These test cases were also used to validate the solvers applied in the present study (Breuer et al., 2012). The laminar benchmarks proposed above are all purely numerical, i.e., a cross-comparison between different numerical results is possible, but no rigorous validation against experimental measurements can be carried out.

In order to close this gap, a nominally 2D laminar experimental case was provided by Gomes and Lienhart (2006, 2013) and Gomes (2011): A very thin metal sheet with an additional weight at the end is attached behind a rotating circular cylinder and mounted inside a channel filled with a mixture of polyglycol and water to reach a low Reynolds number in the laminar regime. Experimental data are provided for several inflow velocities and two different swiveling motions could be identified depending on the inflow velocity. Owing to the thin metal sheet and the rear mass the accurate prediction of this case is demanding. A first comparison between this laminar benchmark and numerical simulations can be found in Gomes et al. (2011): two configurations with different inflow velocities were taken into account. The FSI code is composed of FASTEST-3D (see Section 4.1) for the CFD side and of FEAP (Taylor, 2002) for the CSD side. The results show a very good agreement for the configuration with the higher inflow velocity (second swiveling FSI mode). Nevertheless, differences were observed for the low inflow velocity leading to the first swiveling FSI mode. Gomes et al. (2011) explained these deviations by the influence of the structural damping: in the high inflow velocity case the relevant frequency for the excitation process is the frequency of the coupled system (motioninduced excitation (MIE), see Naudascher and Rockwell (1994)). In the low inflow velocity case, the relevant frequency for the excitation process is the first natural frequency of the pure structure surrounded by vacuum (instability-induced excitation (IIE), see Naudascher and Rockwell (1994)). Thus as argued by Gomes et al. (2011), for the first swiveling mode the FSI phenomenon is more sensitive to the structural damping, which was not considered in the numerical model.

The second category in the classification of FSI benchmarks presented here is composed of test cases based on turbulent flows involving 2D structures: In Gomes et al. (2010) a rigid plate with a single rotational degree of freedom was mounted into a water channel and experimentally studied by particle-image velocimetry (PIV). This study also presents the first comparison between experimental data and predicted results achieved by the present code for a turbulent FSI problem. As another turbulent experimental benchmark, the investigations of Gomes and Lienhart $(2010,2013)$ and Gomes (2011) have to be cited: the same geometry as in Gomes and Lienhart (2006) was used, but this time with water as the working fluid leading to much higher 
Reynolds numbers within the turbulent regime. The resulting FSI test case was found to be very challenging from the numerical point of view. Indeed, the prediction of the deformation and motion of the very thin flexible structure requires two-dimensional finite-elements. On the other hand the discretization of the extra weight mounted at the end of the thin metal sheet calls for three-dimensional volume elements. Thus for a reasonable prediction of this test case both element types have to be used concurrently and have to be coupled adequately. Additionally, the rotational degree of freedom of the front cylinder complicates the structural simulation and the grid adaptation of the flow prediction.

Thus, in the present study a slightly different configuration is considered to provide in a first step a less ambitious test case for the comparison between predictions and measurements focusing the investigations more to the turbulent flow regime and its coupling to a less problematic structural model. For this purpose, a fixed cylinder with a thicker rubber tail and without a rear mass is used. This should open the computation of the proposed benchmark case to a broader spectrum of codes and facilitates its adoption in the community. Strong emphasis is put on a precise description of the experimental measurements, a comprehensive discussion of the modeling in the numerical simulation (for the single field solutions as well as for the coupled problem) and the processing of the respective data to guarantee a reliable reproduction of the proposed test case with various suitable methods.

The paper is organized as follows: A detailed description of this new test case is given in Section 2. The measuring techniques used in the experiment are described in Section 3. Then, the numerical simulation methodology will be presented in Section 4 including a brief resume of the theory of the multi-physics code. Afterwards the full numerical setup is explained. Due to cycle-to-cycle variations in the FSI phenomenon observed in the experiment and in the simulation, the results have to be phase-averaged prior to a detailed comparison. The process is described in Section 5. The experimental unsteady raw results are briefly presented in Section 6. Finally, numerical and experimental phased-resolved results are compared and discussed in Section 7. All data available for comparison are specified in Section 8. For the sake of clarity, the investigations on the material and on the structural model have been relegated to Appendices at the end of the paper.

\section{Description of the Validation Test Case}

\subsection{Description of the geometrical model and the test section}

The proposed benchmark case, denoted FSI-PfS-1a, is derived from the turbulent benchmark of Gomes and Lienhart $(2010,2013)$. In their test case a very thin metal sheet with an additional weight at the end was attached behind a rotating cylinder. The case was found to be very challenging from the point of view of modeling and simulation. Therefore, the idea of the present paper is to propose a simpler FSI benchmark avoiding the aforementioned complicated features and being similar to the recently used FSI test case applied for LES studies (Breuer et al., 2012), but supplemented by experimental data to compare with.

FSI-PfS-1a consists of a flexible thin structure with a distinct thickness clamped behind a fixed rigid non-rotating cylinder installed in a water channel (see Fig. 1). The cylinder has a diameter $D=0.022 \mathrm{~m}$. It is positioned in the middle of the experimental test section with $H_{c}=H / 2=0.120 \mathrm{~m}\left(H_{c} / D \approx 5.45\right)$, whereas the test section denotes a central part of the entire water channel (see Fig. 2). Its center is located at $L_{c}=0.077 \mathrm{~m}\left(L_{c} / D=3.5\right)$ downstream of the inflow section. The test section has a length of $L=0.338 \mathrm{~m}(L / D \approx 15.36)$, a height of $H=0.240 \mathrm{~m}(H / D \approx 10.91)$ and a width $W=0.180 \mathrm{~m}(W / D \approx 8.18)$. The blocking ratio of the channel is about $9.2 \%$. The gravitational acceleration $g$ points in $\mathrm{x}$-direction (see 
Fig. 1), i.e. in the experimental setup this section of the water channel is turned 90 degrees. The deformable structure used in the experiment behind the cylinder has a length $l=0.060 \mathrm{~m}$ $(l / D \approx 2.72)$ and a width $w=0.177 \mathrm{~m}(w / D \approx 8.05)$. Therefore, in the experiment there is a small gap of about $1.5 \times 10^{-3} \mathrm{~m}$ between the side of the deformable structure and both lateral channel walls. The thickness of the rubber plate is $h=0.0021 \mathrm{~m}(h / D \approx 0.09)$. This thickness is an averaged value. The material is natural rubber and thus it is difficult to produce a perfectly homogeneous $2 \mathrm{~mm}$ plate. The measurements show that the thickness is between 0.002 and $0.0022 \mathrm{~m}$. All parameters of the geometrical configuration of the FSI-PfS-1a benchmark are summarized in Table 1.

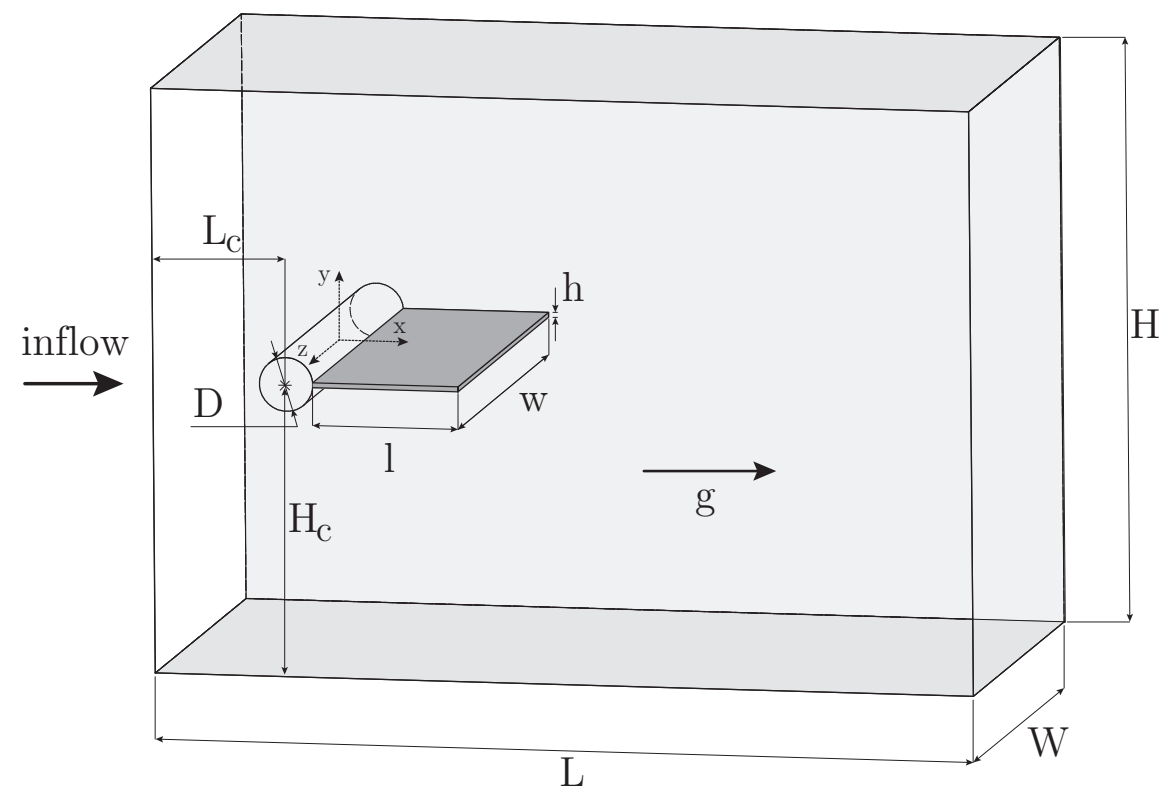

Figure 1: Sketch of the geometrical configuration of the validation test case within the test section.

\begin{tabular}{lll}
\hline Cylinder diameter & $D=0.022 \mathrm{~m}$ & \\
Cylinder center x-position & $L_{c}=0.077 \mathrm{~m}$ & $L_{c} / D=3.5$ \\
Cylinder center y-position & $H_{c}=H / 2=0.120 \mathrm{~m}$ & $H_{c} / D \approx 5.45$ \\
Test section length & $L=0.338 \mathrm{~m}$ & $L / D \approx 15.36$ \\
Test section height & $H=0.240 \mathrm{~m}$ & $H / D \approx 10.91$ \\
Test section width & $W=0.180 \mathrm{~m}$ & $W / D \approx 8.18$ \\
Deformable structure length & $l=0.060 \mathrm{~m}$ & $l / D \approx 2.72$ \\
Deformable structure height & $h=0.0021 \mathrm{~m}$ & $h / D \approx 0.09$ \\
Deformable structure width & $w=0.177 \mathrm{~m}$ & $w / D \approx 8.05$ \\
\hline
\end{tabular}

Table 1: Geometrical configuration of the FSI-PfS-1a validation test case.

\subsection{Description of the water channel}

In order to validate numerical FSI simulations based on reliable experimental data, the special research unit on FSI (Bungartz et al., 2010) designed and constructed a water channel (Göttingen type, see Fig. 2) for corresponding experiments with a special concern regarding controllable and precise boundary and working conditions (Gomes and Lienhart, 2006, 2010; Gomes, 2011). The channel $\left(2.8 \mathrm{~m} \times 1.3 \mathrm{~m} \times 0.5 \mathrm{~m}\right.$, fluid volume of $\left.0.9 \mathrm{~m}^{3}\right)$ has a rectangular 
flow path and includes several rectifiers and straighteners to guarantee a uniform inflow into the test section. To allow optical flow measurement systems like particle-image velocimetry, the test section is optically accessible on three sides. It possesses the same geometry as the test section described in Section 2.1. The structure is fixed on the backplate of the test section and additionally fixed in the front glass plate. With a $24 \mathrm{~kW}$ axial pump a water inflow of up to $u_{\max }=6 \mathrm{~m} / \mathrm{s}$ is possible. To prevent asymmetries the gravity force is aligned with the $\mathrm{x}$-axis in main flow direction.
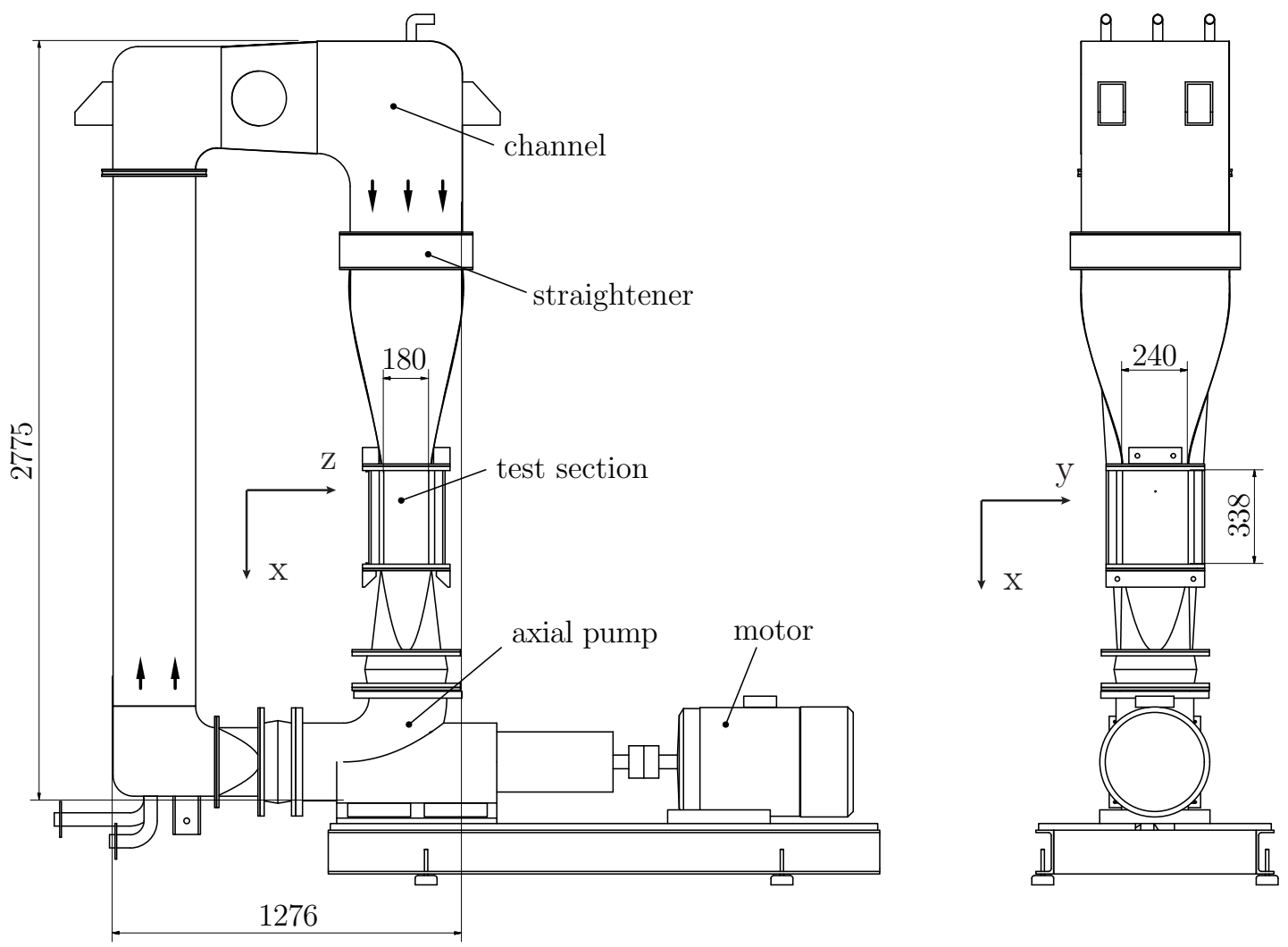

Figure 2: Sketch of the flow channel (dimensions given in $\mathrm{mm}$ ).

\subsection{Flow parameters}

Several preliminary tests were performed to find the best working conditions in terms of maximum structure displacement, good reproducibility and measurable structure frequencies within the turbulent flow regime. Figure 3 depicts the measured extrema of the structure displacement versus the inlet velocity and Figure 4 gives the frequency and Strouhal number of the FSI phenomenon as a function of the inlet velocity. These data were achieved by measurements with the laser distance sensor explained in Section 3.2. The entire diagrams are the result of a measurement campaign in which the inflow velocity was consecutively increased from 0 to $2.5 \mathrm{~m} / \mathrm{s}$. Four regions can be detected (see Fig. 3):

- $u_{\text {inflow }} \leq 0.4 \mathrm{~m} / \mathrm{s}$ : The deflections of the flexible structure are marginal resulting from fluid turbulence fluctuations. This is typical extraneously-induced excitation (EIE) mechanism as explained by Naudascher and Rockwell (1994). 


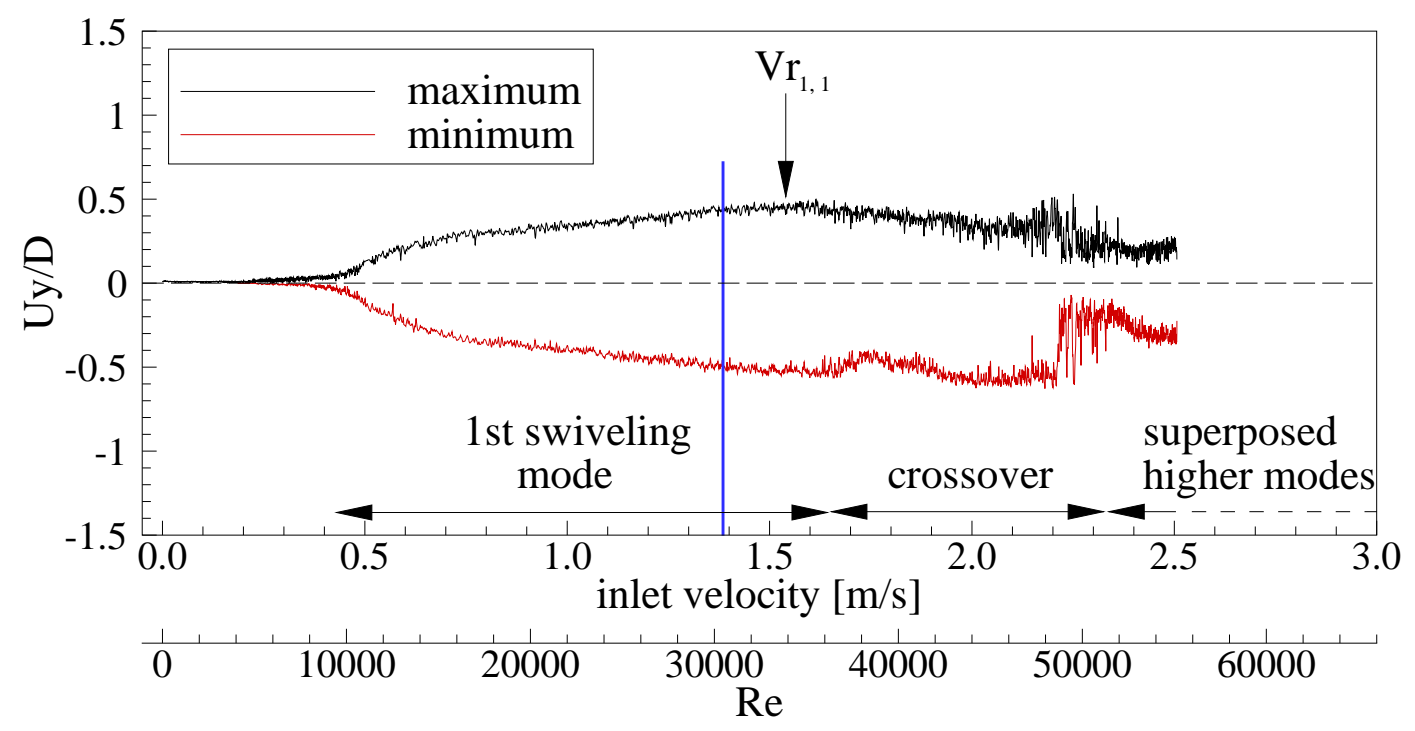

Figure 3: Experimental displacements of the structure extremity versus the inlet velocity.

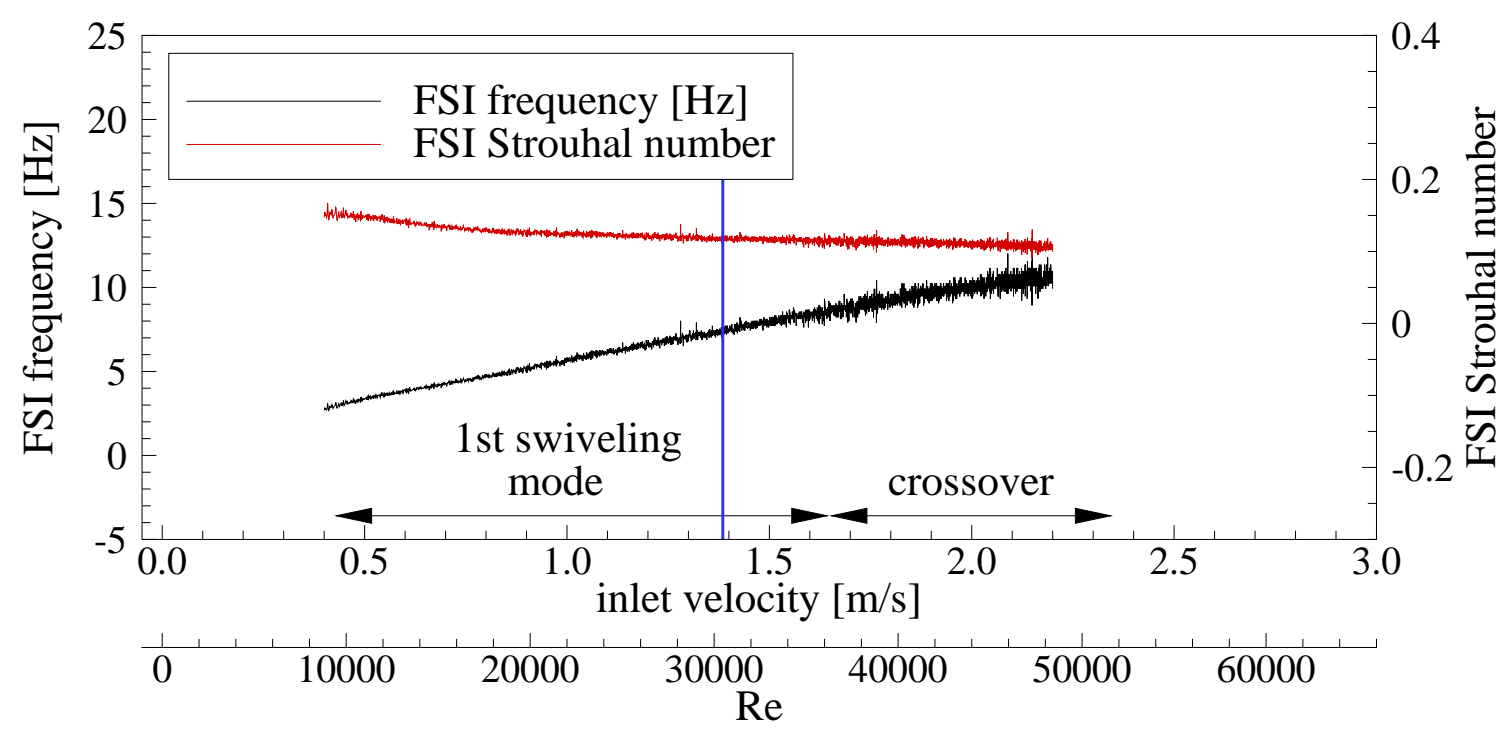

Figure 4: Experimental measurements of the frequency and the corresponding Strouhal number of the FSI phenomenon versus the inlet velocity.

- $0.4 \mathrm{~m} / \mathrm{s}<u_{\text {inflow }} \leq 1.65 \mathrm{~m} / \mathrm{s}$ : The rubber plate deformations are quasi two-dimensional and in the first swiveling mode. This region is dominated by an instability-induced excitation (IIE) (Naudascher and Rockwell, 1994). IIE is provoked by flow instability which gives rise to flow fluctuations if a specific flow velocity is reached. These fluctuations and the resulting forces become well correlated and their frequency is close to a natural frequency of the flexible structure (lock-in phenomenon). In this case oscillations with large amplitudes are expected. Here, the amplitudes increase until the maximum is reached at $u_{\text {inflow }} \approx 1.54$, which corresponds to the reduced velocity $\mathrm{Vr}_{1,1}^{\mathrm{IIE}} \approx 5.71$. The reduced velocity for IIE is defined as follows: $\mathrm{Vr}_{N, n}^{\mathrm{IIE}}=u_{\text {inflow }} /\left(f_{N} D\right) \approx 1 /(n \mathrm{St}) . f_{N}$ is a natural frequency of the flexible structure and St the Strouhal number defined with the vortex-shedding frequency around the undeformed body. The natural frequencies are determined by a modal analysis: The first frequency of the rubber plate $f_{1}$ is found at about 
$12.3 \mathrm{~Hz}$ and corresponds to the first swiveling mode dominant in the FSI phenomenon. St is about 0.175 as specified in Section 7 .

- $1.65 \mathrm{~m} / \mathrm{s}<u_{\text {inflow }} \leq 2.33 \mathrm{~m} / \mathrm{s}$ : In this range the structural deflections are chaotic and three-dimensional. This is a crossover phase. The frequency of the FSI phenomenon increases until the first natural frequency of the rubber plate. Beyond this value it is difficult to measure the FSI frequency because of the chaotic movement.

- $2.33 \mathrm{~m} / \mathrm{s}<u_{\text {inflow }}$ : The deformations observed are three-dimensional and several modes are superposed.

At an inflow velocity of $u_{\text {inflow }}=1.385 \mathrm{~m} / \mathrm{s}$ the displacements are symmetrical, reasonably large and well reproducible. Based on the inflow velocity chosen and the cylinder diameter the Reynolds number of the experiment is equal to $R e=30,470$. Regarding the flow around the front cylinder, at this inflow velocity the flow is in the sub-critical regime. That means the boundary layers are still laminar, but transition to turbulence takes place in the free shear layers evolving from the separated boundary layers behind the apex of the cylinder. Transition to turbulence means that from that point onwards the flow is three-dimensional and chaotic, and consists of a variety of different length and time scales. The low-frequency components of the turbulent flow dominate the coupled FSI problem, whereas the high-frequency contributions are visible in the fluid forces but are filtered out by the flexible structure. That is the reason why the signals for the deflections show the quasi-periodic signals without high-frequency fluctuations as will be shown below in Fig. 11.

Except the boundary layers at the section walls the inflow was found to be nearly uniform in y- and z-direction (see Fig. 5). The time-averaged velocity components $\bar{u}$ and $\bar{v}$ are measured with two-component laser-Doppler velocimetry (LDV) along the y-axis in the middle of the measuring section at $x / D=4.18$ and $z / D=0$. It can be assumed that the time-averaged velocity component $\bar{w}$ shows a similar velocity profile as $\bar{v}$. Furthermore, a low inflow turbulence level of $\mathrm{Tu}_{\text {inflow }}=\sqrt{\frac{1}{3}\left(\overline{u^{\prime 2}}+\overline{v^{\prime 2}}+\overline{w^{\prime 2}}\right)} / u_{\text {inflow }}=0.02$ is measured. All experiments were performed with water under standard conditions at $T=20^{\circ} \mathrm{C}$. The flow parameters are summarized in Table 2.

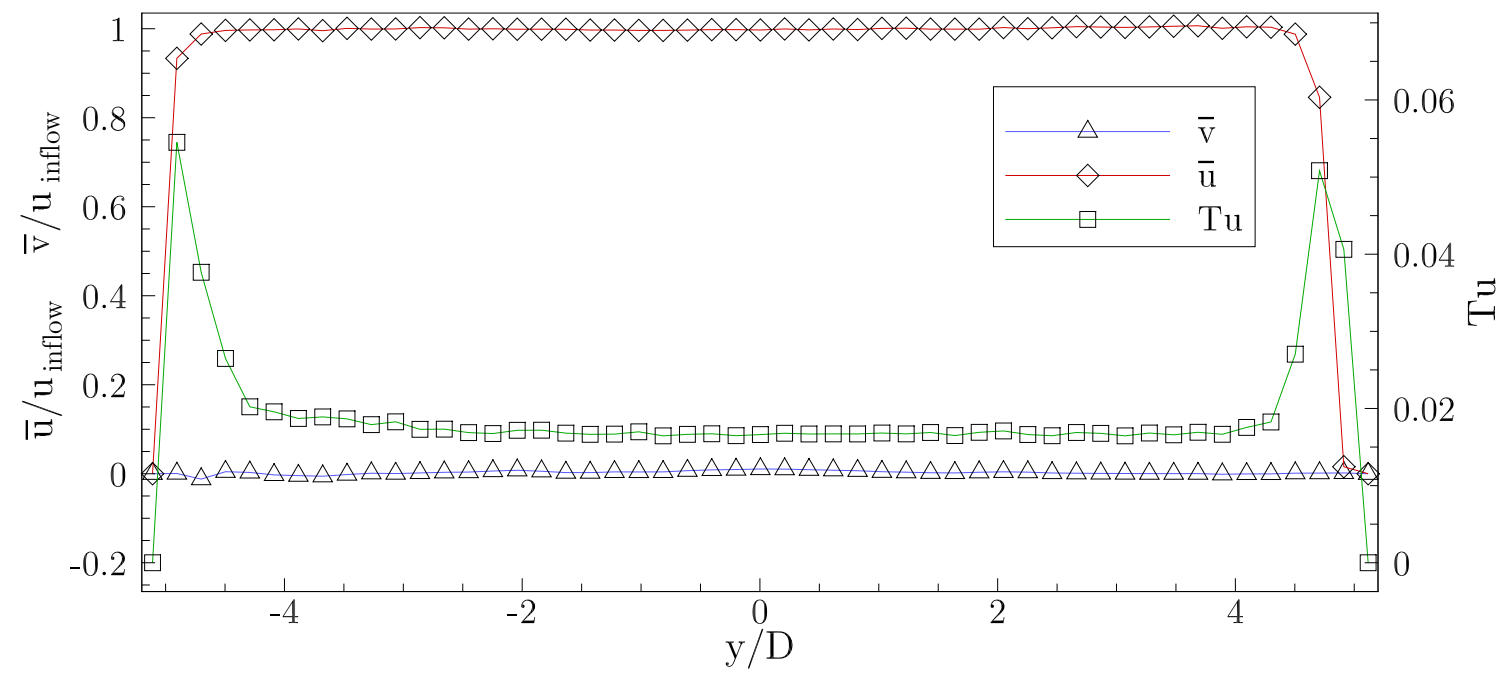

Figure 5: Profiles of the time-averaged streamwise and normal velocity as well as the turbulence level at the inflow section of the water channel for the y-direction. 


\begin{tabular}{ll}
\hline Inflow velocity & $u_{\text {inflow }}=1.385 \mathrm{~m} / \mathrm{s}$ \\
Flow density & $\rho_{f}=1000 \mathrm{~kg} / \mathrm{m}^{3}$ \\
Flow dynamic viscosity & $\mu_{f}=1.0 \times 10^{-3} \mathrm{~Pa} \mathrm{~s}$ \\
\hline
\end{tabular}

Table 2: Flow parameters of the FSI-PfS-1a validation test case.

\subsection{Choice of structural models and material parameters}

Rubber materials are widely used in many different applications, due to, e.g., their isotropic mechanical behavior, their wide range of usable elastic deformations and their adaptability to different needs. Depending on the specific application, the suitable description of the mechanical behavior must be realized by a more or less complex material model such as Ogden, Neo-Hooke, Mooney-Rivlin or Varga (Holzapfel, 2000). For the present FSI benchmark, the material and dimensions of the deformable structure should be selected in such a way that it is rather easy to excite the structure with only moderate fluid forces in the experiment (i.e., it should not be too stiff) and it should undergo only reversible, i.e. elastic, deformations in the range of interest. Moreover, to enable the computation of this test case by many other groups, the structural setup should be simple in order to be described by as few parameters as possible and the structural analysis should be feasible with even less sophisticated material laws like, e.g., St. Venant-Kirchhoff. Another issue is the wish to keep the structural modeling open to either solid or shell finite element formulations which makes it necessary to avoid an excessively thin structure to guarantee the desired flexibility. As a consequence of all these requirements, a custom-made flexible and isotropic rubber material with a reasonable thickness, produced by the company Draftex Automotive GmbH, is applied and its material parameters are presented below. For understanding the behavior of the rubber used for the structural model and to verify the characteristic parameters for the structural simulations, pure structural test cases were defined and performed in the laboratory (See Appendix A).

Although the material shows a strong non-linear elastic behavior for large strains, the application of a linear elastic constitutive law is favored, to enable the reproduction of this FSI benchmark by a variety of different computational analysis codes without the need of complex material laws. This assumption can be justified by the observation that in the FSI test case, a formulation for large deformations but small strains is applicable. Hence, the identification of the material parameters is done on the basis of the moderate strain expected and the St. Venant-Kirchhoff constitutive law is chosen as the simplest hyper-elastic material model.

The density of the rubber material can be determined to be $\rho_{\text {rubber plate }}=1360 \mathrm{~kg} / \mathrm{m}^{3}$ for a thickness of the plate $h=0.0021 \mathrm{~m}$. This permits the accurate modeling of inertia effects of the structure and thus dynamic test cases can be used to calibrate the material constants. For the chosen material model there are only two parameters to be defined: The Young's modulus $E$ and the Poisson's ratio $\nu$. In order to avoid complications in the needed element technology due to incompressibility, the material was realized to have a Poisson's ratio which reasonably differs from 0.5. Material tests of the manufacturer indicate that the Young's modulus is $E=16 \mathrm{MPa}$ and the Poisson's ratio is $\nu=0.48$. The material parameters are summarized in Table 3.

To numerically validate the decision on structural models and to check the material parameters simulations are carried out with the reference software Abaqus ${ }^{1}$ on the pure structural test cases

\footnotetext{
${ }^{1}$ http://www.3ds.com/products/simulia/portfolio/abaqus/overview
} 


\begin{tabular}{lll}
\hline Flexible structure density & $\rho_{\text {rubber plate }}=1360 \mathrm{~kg} / \mathrm{m}^{3}$ \\
Young's modulus & $E$ & $=16 \mathrm{MPa}$ \\
Poisson's ratio & $\nu$ & $=0.48$ \\
\hline
\end{tabular}

Table 3: Structural parameters of the FSI-PfS-1a flexible structure.

described in Appendix A. The results are presented in Appendix B.

\section{Measuring Techniques for the Experimental Investigations}

Experimental FSI investigations need to contain fluid and structure measurements for a full description of the coupling process. Under certain conditions, the same technique for both disciplines can be used. The measurements performed by Gomes and Lienhart (2006, 2010, 2013) used the same camera system for the simultaneous acquisition of the velocity fields and the structural deflections. This procedure works well for FSI cases involving laminar flows and 2D structure deflections. In the present case the structure deforms slightly three-dimensional with increased cycle-to-cycle variations caused by turbulent variations in the flow. The applied measuring techniques, especially for the structural side, have to deal with those changed conditions especially the formation of shades. Furthermore, certain spatial and temporal resolutions as well as low measurement errors are requested. Due to the different deformation behavior a single camera setup for the structural measurements like in Gomes and Lienhart $(2006,2010,2013)$ used was not practicable. Therefore, the velocity fields were captured by a 2D particle-image velocimetry (PIV) setup and the structural deflections were measured with a laser triangulation technique. Both devices are presented in the next sections.

\subsection{Particle-image velocimetry}

A classic particle-image velocimetry (Adrian, 1991) setup depicted in Fig. 6 consists of a single camera obtaining two components of the fluid velocity on a planar surface illuminated by a laser light sheet. Particles introduced into the fluid are following the flow and reflecting the light during the passage of the light sheet. By taking two reflection fields in a short time interval $\Delta t$, the most-likely displacements of several particle groups on an equidistant grid are estimated by a cross-correlation technique or a particle-tracking algorithm. Based on a precise preliminary calibration, the displacements obtained and the time interval $\Delta t$ chosen the velocity field can be calculated. To prevent shadows behind the flexible structure a second light sheet was used to illuminate the opposite side of the test section.

The phased-resolved PIV-measurements (PR-PIV) were carried out with a 4 Mega-pixel camera (TSI Powerview 4MP, charge-coupled device (CCD) chip) and a pulsed dual-head Neodym:YAG laser (Litron NanoPIV 200) with an energy of $200 \mathrm{~mJ}$ per laser pulse. The high energy of the laser allowed to use silver-coated hollow glass spheres (SHGS) with an average diameter of $d_{\text {avg,SHGS }}=10 \mu \mathrm{m}$ and a density of $\rho_{S H G S}=1400 \mathrm{~kg} / \mathrm{m}^{3}$ as tracer particles. To prove the following behavior of these particles the Stokes number Sk and the particle sedimentation velocity $u_{\text {SHGS }}$ is calculated as follows:

$$
\begin{aligned}
S k_{\mathrm{SHGS}} & =\frac{\tau_{\mathrm{p}, \mathrm{SHGS}}}{\tau_{\mathrm{f}, \mathrm{SHGS}}}=\frac{\rho_{\mathrm{SHGS}} d_{\mathrm{avg}, \mathrm{SHGS}}^{2}}{18 \mu_{f}} \frac{u_{\text {inflow }}}{d_{\mathrm{avg}, \mathrm{SHGS}}}=1.08, \\
u_{\mathrm{SHGS}} & =\frac{d_{\mathrm{avg}, \mathrm{SHGS}}^{2} g\left(\rho_{\mathrm{SHGS}}-\rho_{f}\right)}{18 \mu_{f}}=2.18 \times 10^{-5} \mathrm{~m} / \mathrm{s} .
\end{aligned}
$$




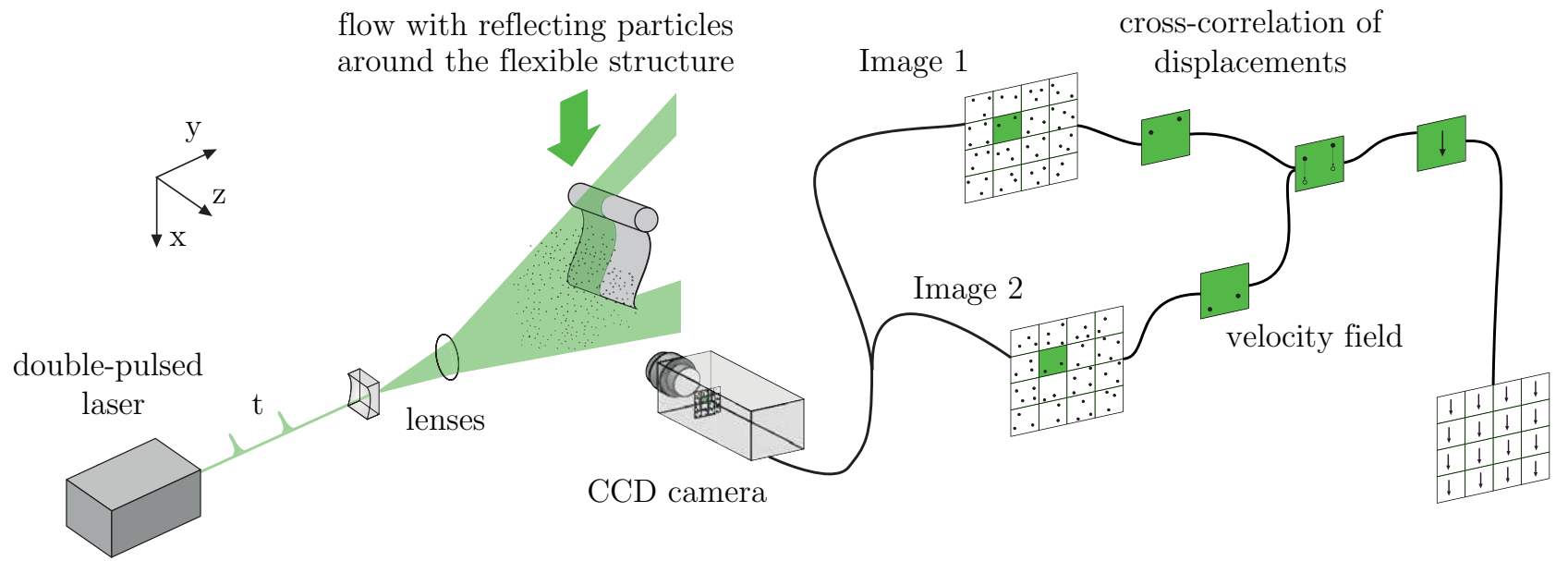

Figure 6: Measuring principle of a two-component PIV setup for the flow around the flexible structure.

With this Stokes number and a particle sedimentation velocity which is much lower than the expected velocities in the experiments, an eminent following behavior is approved. The camera takes 12 bit pictures with a frequency of about $7.0 \mathrm{~Hz}$ and a resolution of $1695 \times 1211 \mathrm{px}$ with respect to the rectangular size of the test section. For one phase-resolved position (described in Section 5) 60 to 80 measurements are taken. Preliminary studies with more and fewer measurements showed that this number of measurements represent a good compromise between accuracy and effort. The grid has a size of $150 \times 138$ cells and was calibrated with an average factor of $126 \mu \mathrm{m} / \mathrm{px}$, covering a planar flow field of $x / D \approx-2.36$ to 7.26 and $y / D \approx-3.47$ to 3.47 in the middle of the test section at $z / D \approx 0$. The time between the frame-straddled laser pulses was set to $\Delta t=200 \mu \mathrm{s}$. Laser and camera were controlled by a TSI synchronizer (TSI 610035) with 1 ns resolution. The processing of the phase-resolved fluid velocity fields involving the structure deflections is described in Section 5.

\subsection{Laser distance sensor}

Non-contact structural measurements are often based on laser distance techniques. In the present benchmark case the flexible structure shows an oscillating frequency of about $7.1 \mathrm{~Hz}$. With the requirement to perform more than 100 measurements per period, a time-resolved system was needed. Therefore, a laser triangulation was chosen because of the known geometric dependencies, the high data rates, the small measurement range and the resulting higher accuracy in comparison with other techniques such as laser phase-shifting or laser interferometry. The laser triangulation uses a laser beam which is focused onto the object. A CCD-chip located near the laser output detects the reflected light on the object surface. If the distance of the object from the sensor changes, also the angle changes and thus the position of its image on the CCD-chip. From this change in position the distance to the object is calculated by simple trigonometric functions and an internal length calibration adjusted to the applied measurement range. To study simultaneously more than one point on the structure, a multiple-point triangulation sensor was applied (Micro-Epsilon scanControl 2750, see Fig. 7). This sensor uses a matrix of CCD chips to detect the displacements on up to 640 points along a laser line reflected on the surface of the structure with a data rate of 800 profiles per second. The laser line was positioned in a horizontal $(x / D \approx 2.82$, see Fig. $7(\mathrm{a}))$ and in a vertical alignment $(z / D \approx 0$, see Fig. $7(\mathrm{~b}))$ and has an accuracy of $40 \mu \mathrm{m}$. Due to the different refraction indices of air, glass and water a custom calibration was performed to take the modified optical behavior of the 
emitted laser beams into account.

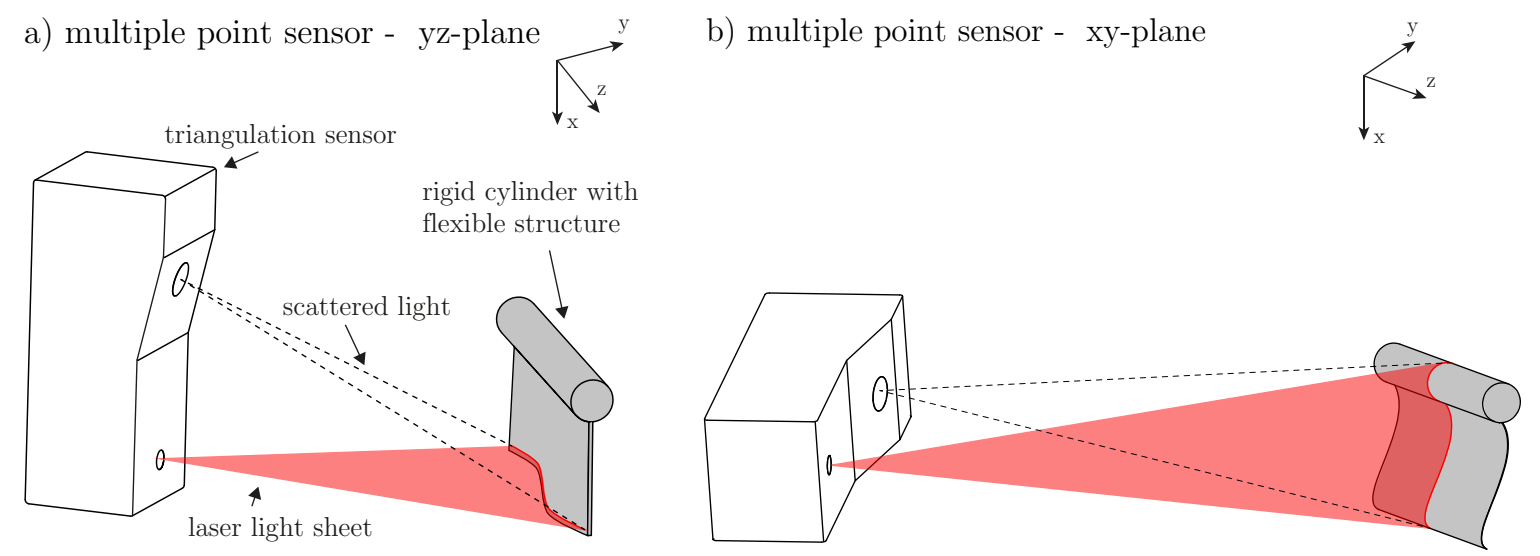

Figure 7: Setup and alignment of multiple-point laser sensor on the flexible structure in a) z-direction and b) x-direction.

\section{Numerical Simulation Methodology}

The applied numerical method relies on an efficient partitioned coupling scheme developed for dynamic fluid-structure interaction problems in turbulent flows (Breuer et al., 2012). The fluid flow is predicted by an eddy-resolving scheme, i.e., the large-eddy simulation technique. FSI problems very often encounter instantaneous non-equilibrium flows with large-scale flow structures such as separation, reattachment and vortex shedding. For this kind of flows the LES technique is obviously the best choice (Breuer, 2002). Based on a semi-implicit scheme the LES code is coupled with a code especially suited for the prediction of shells and membranes. Thus an appropriate tool for the time-resolved prediction of instantaneous turbulent flows around light, thin-walled structures results. Since all details of this methodology were recently published in Breuer et al. (2012), in the following only a brief description is provided.

\subsection{Computational fluid dynamics (CFD)}

In the present methodology the temporally varying domain within a FSI application is taken into account by the Arbitrary Lagrangian-Eulerian (ALE) formulation. The application of the ALE method is limited to FSI cases with mild or moderate deformations of the structure. For extreme deflections other techniques such as the immersed boundary method or overset grids should be applied. However, since in the current test case this restriction is satisfied and the ALE method allows to adequately resolve the thin boundary layers on the structure using curvilinear body-fitted grids without artificial boundary conditions, it is favored for the present application.

The extra fluxes appearing in the filtered Navier-Stokes equations are consistently determined by the space conservation law (SCL) (Demirdžić and Perić, 1988, 1990; Lesoinne and Farhat, 1996). For this purpose the in-house code FASTEST-3D (Durst and Schäfer, 1996; Durst et al., 1996) relying on a finite-volume scheme is used. The discretization on a block-structured bodyfitted grid is second-order accurate in space.

A predictor-corrector scheme (projection method) of second-order accuracy forms the kernel of the fluid solver. In the predictor step an explicit Runge-Kutta scheme advances the momentum equation in time. In the following corrector step the mass conservation equation is fulfilled by 
solving a Poisson equation for the pressure-correction. The corrector step is repeated until a predefined convergence criterion is reached.

In LES the large scales are resolved directly, whereas the non-resolvable small scales have to be taken into account by a subgrid-scale model. Here, the well-known Smagorinsky (1963) model is applied. A Van Driest damping function ensures a reduction of the subgrid length near solid walls. Owing to minor influences of the subgrid-scale model at the moderate Reynolds number considered in this study, a dynamic procedure to determine the Smagorinsky parameter was omitted and instead a well established standard constant $C_{s}=0.1$ is used.

\subsection{Computational structural dynamics (CSD)}

The dynamic equilibrium of the structure is described by the momentum equation given in a Lagrangian frame of reference. Large deformations, where geometrical non-linearities are not negligible, are allowed (Hojjat et al., 2010). According to the preliminary considerations described in Section 2.4, a total Lagrangian formulation in terms of the second Piola-Kirchhoff stress tensor and the Green-Lagrange strain tensor which are linked by the St. Venant-Kirchhoff material law is used in the present study. The investigations within this paper were done with the in-house code Carat++ (Fischer et al., 2010; Bletzinger et al., 2006), developed with an emphasis on the prediction of shell or membrane behavior. Carat++ is based on several finite-element types and advanced solution strategies for form finding and non-linear dynamic problems (Wüchner and Bletzinger, 2005; Wüchner et al., 2007; Bletzinger et al., 2005; Dieringer et al., 2012). For the dynamic analysis, different time-integration schemes are available, e.g., the implicit generalized- $\alpha$ method (Chung and Hulbert, 1993). In the modeling of thin-walled structures, membrane or shell elements are applied. The deformable solid is modeled with a 7-parameter shell element. Furthermore, special care is given to prevent locking phenomena by applying the well-known Assumed Natural Strain (ANS) (Hughes and Tezduyar, 1981; Park and Stanley, 1986) and Enhanced Assumed Strain (EAS) methods (Bischoff et al., 2004).

Both, shell and membrane elements reflect geometrically reduced structural models with a two-dimensional representation of the mid-surface which can describe the three-dimensional physical properties by introducing mechanical assumptions for the thickness direction. Due to this reduced model additional operations are required to transfer information between the two-dimensional structure and the three-dimensional fluid model. Thus in the case of shells, the surface of the interface is found by moving the two-dimensional surface of the structure half of the thickness normal to the surface on both sides and the closing of the volume (Bletzinger et al., 2006). On these two moved surfaces the exchange of data is performed consistently with respect to the shell theory (Hojjat et al., 2010).

\subsection{Coupling algorithm}

To preserve the advantages of the highly adapted CSD and CFD codes and to realize an effective coupling algorithm, a partitioned but nevertheless strong coupling approach is chosen. The scheme involves an explicit solution of the non-linear terms in the Navier-Stokes equations and an implicit coupling between the computation of the pressure field and the displacements of the structure. Thus small time steps typically required for LES to resolve the turbulent flow field are taken into account in the coupling scheme relying on this explicit predictor-corrector scheme. Since all details are provided in Breuer et al. (2012), only a few issues forming the kernel of the fluid solver are provided here.

For a flexible structure in water, the added-mass effect by the surrounding fluid plays a dominant role. In this situation a strong coupling scheme taking the tight interaction between the 
fluid and the structure into account, is indispensable. In the coupling scheme developed in Breuer et al. (2012) this issue is taken into account by a FSI-subiteration loop which avoids instabilities due to the added-mass effect known from loose coupling schemes and maintains the explicit character of the time-stepping scheme beneficial for LES.

At the beginning of a time step a prediction of the structural displacement is carried out by a first-order extrapolation to accelerate the convergence. A second-order prediction is not applied, since it was observed that it does not improve the convergence for the current small time-step size.

Based on the velocity and pressure fields from the corrector step, the fluid forces resulting from the pressure and the viscous shear stresses at the interface between the fluid and the structure are computed. These forces are transferred by a grid-to-grid data interpolation to the CSD code Carat++ using a conservative interpolation scheme (Farhat et al., 1998) implemented in the coupling interface CoMA (Gallinger et al., 2009). The conservative interpolation of the forces ensures that the load resultants on both grids are exactly the same. This advantage is accompanied by the drawback that in case of a coarse source grid (CFD) and very fine target grid (CSD), the loads are distributed in a non-physical way. For the present and most other FSI applications this is however not the case, since the grid used for LES is much finer than the CSD grid.

Using the fluid forces provided via CoMA, Carat++ determines the stresses in the structure and the resulting displacements of the structure. This response of the structure is transferred back to the fluid solver via CoMA applying a bilinear interpolation which is a consistent scheme for four-node elements with bilinear shape functions.

The CSD prediction determines displacements at the moving boundaries of the computational domain for the fluid flow. The task is to adapt at each FSI-subiteration the grid of the inner computational domain based on these displacements at the interface. For moderate deformations algebraic methods are found to be a good compromise since they are extremely fast and deliver reasonable grid point distributions maintaining the required high grid quality. Thus, the grid adjustment is performed based on a transfinite interpolation (Thompson et al., 1985). It consists of three shear transformations plus a tensor-product transformation.

The code coupling tool CoMA is based on the Message-Passing-Interface (MPI) and thus runs in parallel to the fluid and structure solver. The communication in-between the codes is performed via standard MPI commands. Since the parallelization in FASTEST-3D and Carat++ also relies on MPI, a hierarchical parallelization strategy with different levels of parallelism is achieved.

For more details about this semi-implicit coupling scheme, we refer to Breuer et al. (2012).

\subsection{Numerical setup}

4.4.1. Grids

CFD prediction.

For the CFD prediction of the flow two different block-structured grids either for a subset of the entire channel $\left(w^{\prime} / l=1\right)$ or for the full channel but without the gap between the flexible structure and the side walls $(w / l=2.95)$ are used (see Fig. 8). In the first case the entire grid consists of about 13.5 million control volumes (CVs), whereas 72 equidistant CVs are applied in the spanwise direction. For the full geometry the grid possesses about 22.5 million CVs. In this case starting close to both channel walls the grid is stretched geometrically with a stretching factor 1.1 applying in total $120 \mathrm{CVs}$ with the first cell center positioned at a distance of $\Delta z / D=1.7 \times 10^{-2}$. 


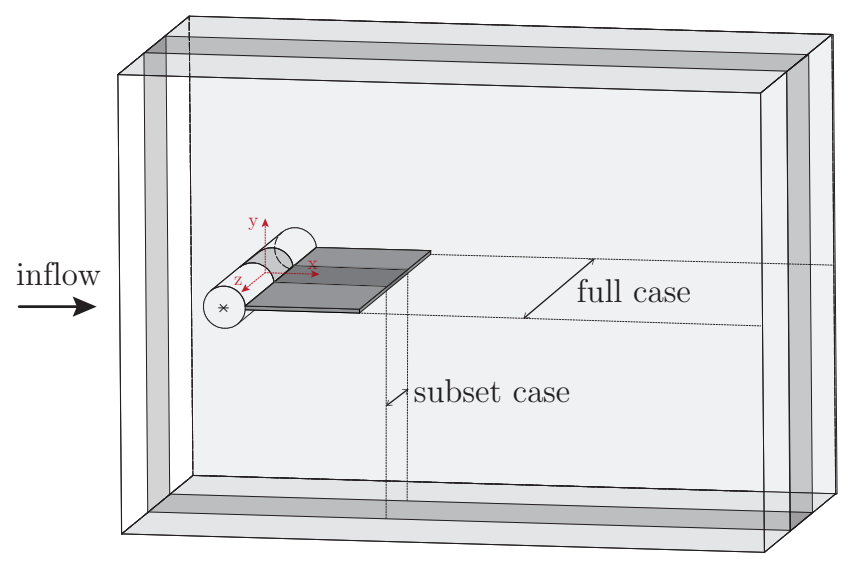

Figure 8: Differences between the full and the subset case.

The gap between the elastic structure and the walls is not taken into account in the numerical model and thus the width of the channel is set to $w$ instead of $W$. Two main reasons are responsible for this simplification. If the gap would be considered in the simulation, the boundary layers of the channel walls had to be fully resolved, which is too costly. Moreover, the cells in this gap would be subjected to heavy distortions during the FSI simulation, which would massively complicate the purpose of grid adaptation during the movement of the flexible structure close to the side walls and may even lead to convergence problems of the coupled solver.

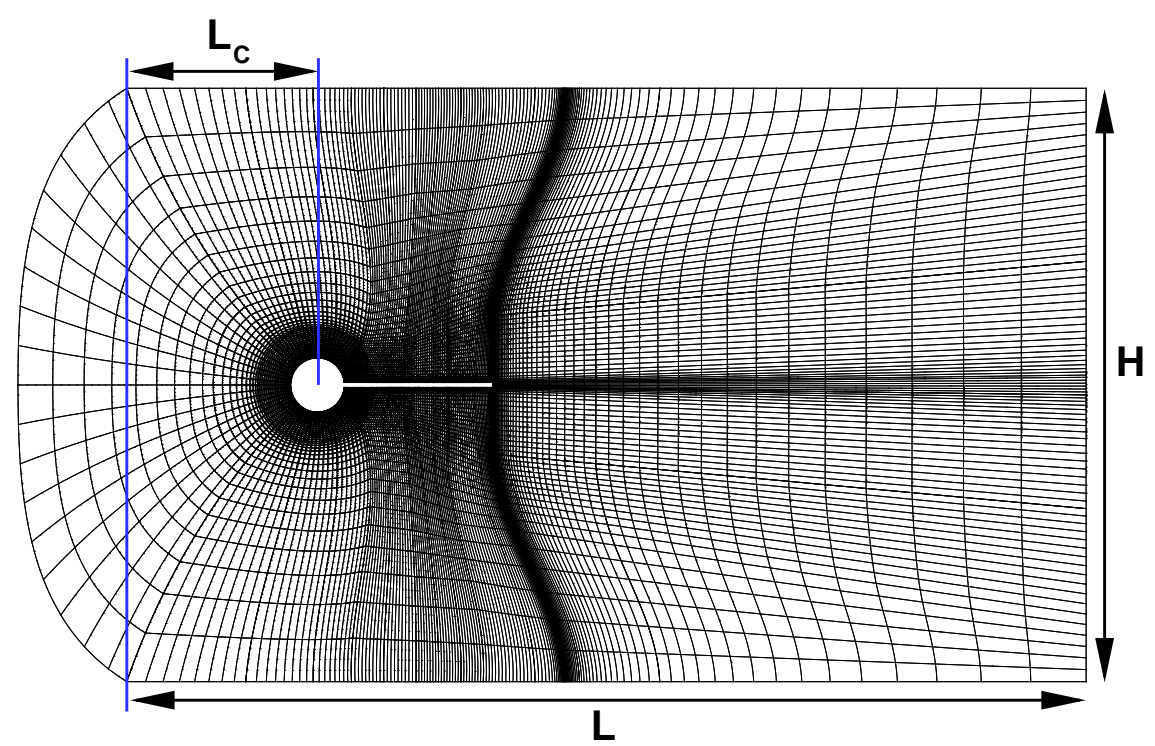

Figure 9: $x-y$ cross-section of the grids used for the simulation with 187,500 cells (Note that only every fourth grid line in each direction is displayed here).

In the $\mathrm{x}-\mathrm{y}$ cross-section both grids are identical (see Fig. 9). Since only every fourth grid line of the mesh is shown in Fig. 9, the angles between grid lines and the transitions between the blocks appear to be worse than in the original grid. The numerical domain has a length of $L$. Since the inflow side is rounded in order to use a C-grid, the computational domain in front of the cylinder is slightly larger than in the test section depicted in Fig. 1. The grid points are clustered towards the rigid cylinder and the flexible structure using a stretching function 
according to a geometric series. The stretching factors are kept below 1.1 with the first cell center located at a distance of $\Delta y / D=9 \times 10^{-4}$ from the flexible structure. Based on the wall shear stresses on the flexible structure the average $y^{+}$values are predicted to be below 0.8 , mostly even below 0.5 . Thus, the viscous sublayer on the elastic structure and the cylinder is adequately resolved. Since the boundary layers at the upper and lower channel walls are not considered, no grid clustering is required here.

\section{CSD prediction.}

Motivated by the fact that in the case of LES frequently a domain modeling based on periodic boundary conditions at the lateral walls is used to reduce the CPU-time requirements, this special approach was also investigated for the FSI test case. The detailed discussion of this specific boundary modeling for the spanwise direction is given in Section 4.4.2. As a consequence, there are two different structure meshes used: For the CSD prediction of the case with a subset of the full channel the elastic structure is resolved by the use of $10 \times 10$ quadrilateral four-node shell elements. For the case discretizing the entire channel, 10 quadrilateral four-node shell elements are used in the main flow direction and 30 in the spanwise direction. These choices are derived from a grid independency study based on a representative pure structure simulation test case with a very similar deformation pattern. This allows to avoid the high computational overhead of fully coupled FSI simulations. The investigations are presented in Appendix C. The finite elements for the structure are 7-parameter shell elements with 6 degrees of freedom per node (Büchter and Ramm, 1992; Büchter et al., 1994; Bischoff and Ramm, 2000). The specific degrees of freedom are the three deformations of the mid-surface and the three components of the difference vector of the shell director. Special treatments for the thickness stretch are included to avoid the undesirable effect which is called thickness locking (Bischoff and Ramm, 1997). For the present test cases the ANS method is not activated and the EAS method is used according to the recommendations in Bischoff et al. (2004) and Bischoff (1999), to have an effective counter-measure against transverse shear-, membrane-, in-plane shear and thickness locking. For a detailed derivation of the element, an in-depth discussion of valid shell formulations and locking phenomena in shell element analysis, the reader is referred to these two studies. The shell elements used are formulated for large deformations, i.e., geometrically non-linear analysis. In the given benchmark scenario, the ratio of the thickness and the length of the thin structure, $h / l=0.035$, is smaller than $1 / 10$. So even a 5 -parameter shell using the Reissner-Mindlin kinematics (Reissner, 1945; Mindlin, 1951) would be valid. The 7-parameter theory yields higher accuracy for the representation of through-the-thickness effects and is for the structure considered fully comparable to a solid model.

\subsubsection{Boundary conditions \\ CFD prediction.}

On the CFD side no-slip boundary conditions are applied at the rigid front cylinder and at the flexible structure. Since the resolution of the boundary layers at the channel walls would require the bulk of the CPU-time, the upper and lower channel walls are assumed to be slip walls. Thus the blocking effect of the walls is maintained without taking the boundary layers into account. At the inlet a constant streamwise velocity is set as inflow condition without adding any perturbations. The choice of zero turbulence level is based on the consideration that such small perturbations imposed at the inlet will generally not reach the cylinder due to the coarseness of the grid at the outer boundaries. Therefore, all inflow fluctuations will be highly damped. However, since the flow is assumed to be sub-critical, this disregard is insignificant. At the outlet a convective outflow boundary condition is favored allowing vortices to leave the 
integration domain without significant disturbances (Breuer, 2002). The convection velocity is set to $u_{\text {inflow }}$.

As mentioned above two different cases are considered (see Fig. 8). In order to save CPUtime in the first case only a subset of the entire spanwise extension of the channel is taken into account. Thus the computational domain has a width of $w^{\prime} / l=1$ in z-direction and the flexible structure is a square in the x-z-plane. In this case a reasonable approximation already applied in Breuer et al. (2012) is to apply periodic boundary conditions in spanwise direction for both disciplines. For LES predictions periodic boundary conditions represent an often used measure in order to avoid the formulation of appropriate inflow and outflow boundary conditions. The approximation is valid as long as the turbulent flow is homogeneous in the specific direction and the width of the domain is sufficiently large. The latter can be proven by predicting two-point correlations, which have to drop towards zero within the half-width of the domain. The impact of periodic boundary conditions on the CSD predictions are discussed below.

For the full case with $w / l=2.95$ periodic boundary conditions can no longer be used. Instead, for the fluid flow similar to the upper and lower walls also for the lateral boundaries slip walls are assumed since the full resolution of the boundary layers would be again too costly. Furthermore, the assumption of slip walls is consistent with the disregard of the small gap between the flexible structure and the side walls discussed above.

\section{CSD prediction.}

On the CSD side, the flexible shell is loaded on the top and bottom surface by the fluid forces, which are transferred from the fluid mesh to the structure mesh. These Neumann boundary conditions for the structure reflect the coupling conditions. Concerning the Dirichlet boundary conditions, the four edges need appropriate support modeling: on the upstream side at the rigid cylinder a clamped support is realized and all degrees of freedom are equal to zero. On the opposite downstream trailing-edge side, the rubber plate is free to move and all nodes have the full set of six degrees of freedom. The edges which are aligned to the main flow direction need different boundary condition modeling, depending on whether the subset or the full case is computed:

For the subset case due to the fluid-motivated periodic boundary conditions, periodicity for the structure is correspondingly assumed for consistency reasons. As it turns out later in Section 7.1.1, this assumption seems to hold for this specific configuration and its deformation pattern which has strong similarity with an oscillation in the first eigenmode of the shell. Hence, this modeling approach may be used for the efficient processing of parameter studies, e.g., to evaluate the sensitivity of the FSI simulations with respect to slight variations in model parameters shown in Section 7.1.2. For this special type of support modeling, there are always two structure nodes on the lateral sides (one in a plane $z=-w / 2$ and its twin in the other plane $z=+w / 2$ ) which have the same load. These two nodes must have the same displacements in $\mathrm{x}$ - and $\mathrm{y}$-direction and their rotations have to be identical. Moreover, the periodic boundary conditions imply that the z-displacement of the nodes on the sides are forced to be zero.

For the full case the presence of the walls in connection with the small gap implies that there is in fact no constraining effect on the structure, as long as no contact between the rubber plate and the wall takes place. Out of precise observations in the lab, the possibility of contact may be disregarded. In principle, this configuration would lead to free-edge conditions like at the trailing edge. However, the simulation of the fluid with a moving mesh needs a well-defined mesh situation at the side walls which made it necessary to tightly connect the structure mesh to the walls (the detailed representation of the side edges within the fluid mesh is discarded due 
to computational costs and the resulting deformation sensitivity of the mesh in these regions). Also the displacement in z-direction of the structure nodes at the lateral boundaries is forced to be zero.

\subsubsection{Coupling conditions}

For the turbulent flow a time-step size of $\Delta t_{f}=2 \times 10^{-5} \mathrm{~s}\left(\Delta t_{f}^{*}=1.26 \times 10^{-3}\right.$ in dimensionless form using $u_{\text {inflow }}$ and $D$ as reference quantities) is chosen and the same time-step size is applied for the structural solver based on the generalized- $\alpha$ method with the spectral radius $\varrho_{\infty}=1.0$, i.e, the Newmark standard method. For the CFD part this time-step size corresponds to a CFL number in the order of unity. Furthermore, a constant underrelaxation factor of $\omega=0.5$ is considered for the displacements and the loads are transferred without underrelaxation. In accordance with previous laminar and turbulent cases in Breuer et al. (2012) the FSI convergence criterion is set to $\varepsilon_{\mathrm{FSI}}=10^{-4}$ for the $\mathrm{L}_{2}$ norm of the displacement differences. 4 to 15 FSI-subiterations are required to reach the convergence criterion. The mean value of the FSIsubiterations for an entire simulation is about 5.8: The maxima are only appearing in short time intervals during the maximum deflections of the structure or when the plate deforms with the maximum velocity.

After an initial phase in which the coupled system reaches a statistically steady state, each simulation is carried out for about $4 \mathrm{~s}$ real-time corresponding to about 27 swiveling cycles of the flexible structure.

For the coupled LES predictions the national supercomputer SuperMIG/SuperMUC was used applying either 82 or 140 processors for the CFD part of the reduced and full geometry, respectively. Additionally, one processor is required for the coupling code and one processor for the CSD code, respectively.

\section{Generation of Phase-resolved Data}

Each flow characteristic of a quasi-periodic FSI problem can be written as a function $f=$ $\bar{f}+\tilde{f}+f^{\prime}$, where $\bar{f}$ describes the global mean part, $\tilde{f}$ the quasi-periodic part and $f^{\prime}$ a random turbulence-related part (Reynolds and Hussain, 1972; Cantwell and Coles, 1983). This splitting can also be written in the form $f=\langle f\rangle+f^{\prime}$, where $\langle f\rangle$ is the phase-averaged part, i.e., the mean at constant phase. In order to be able to compare numerical results and experimental measurements, the irregular turbulent part $f^{\prime}$ has to be averaged out. This measure is indispensable owing to the nature of turbulence which only allows reasonable comparisons based on statistical data. Therefore, the present data are phase-averaged to obtain only the phase-resolved contribution $\langle f\rangle$ of the problem, which can be seen as a representative and thus characteristic signal of the underlying FSI phenomenon.

\subsection{Description of the method}

The procedure to generate phase-resolved results is the same for the experiments and the simulations and is also similar to the one presented in Gomes and Lienhart (2006). The technique can be split up into three steps:

\section{- Reduce the 3D-problem to a 2D-problem}

Due to the facts that in the present benchmark the structure deformation in spanwise direction is negligible and that the delivered experimental PIV-results are only available in one $\mathrm{x}$-y-plane, first the 3D-problem is reduced to a 2D-problem. For this purpose the flow field and the shell position in the CFD predictions are averaged in spanwise direction. 
- Determine $n$ reference positions for the FSI problem

A representative signal of the FSI phenomenon is the history of the y-displacements of the shell extremity. Therefore, it is used as the trigger signal for this averaging method leading to phase-resolved data. Note that the averaged period of this signal is denoted $T$. At first, it has to be defined in how many sub-parts the main period of the FSI problem will be divided and so, how many reference positions have to be calculated (for example in the present work $n=23$ ). Then, the margins of each period of the $\mathrm{y}$-displacement curve are determined. In order to do that the intersections between the y-displacement curve and the zero crossings $\left(U_{y}=0\right)$ are looked for and used to limit the periods. Third, each period $T_{i}$ found is divided into $n$ equidistant sub-parts denoted $j$ (see Fig. 10(a)).

- Sort and average the data corresponding to each reference position

The sub-part $j$ of the period $T_{i}$ corresponds to the sub-part $j$ of the period $T_{i+1}$ and so on. Each data set found in a sub-part $j$ will be averaged with the other sets found in the sub-parts $j$ of all other periods (see Fig. 10(b)). Finally, data sets of $n$ phase-averaged positions for the representative reference period are achieved.

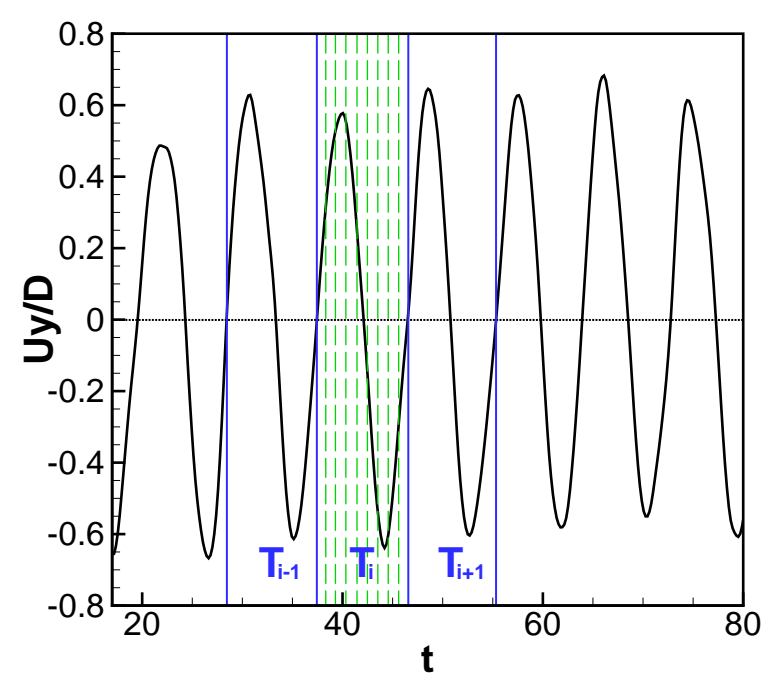

(a) In blue the periods and in green the $n$ equidistant sub-parts of the period $T_{i}$ are shown.

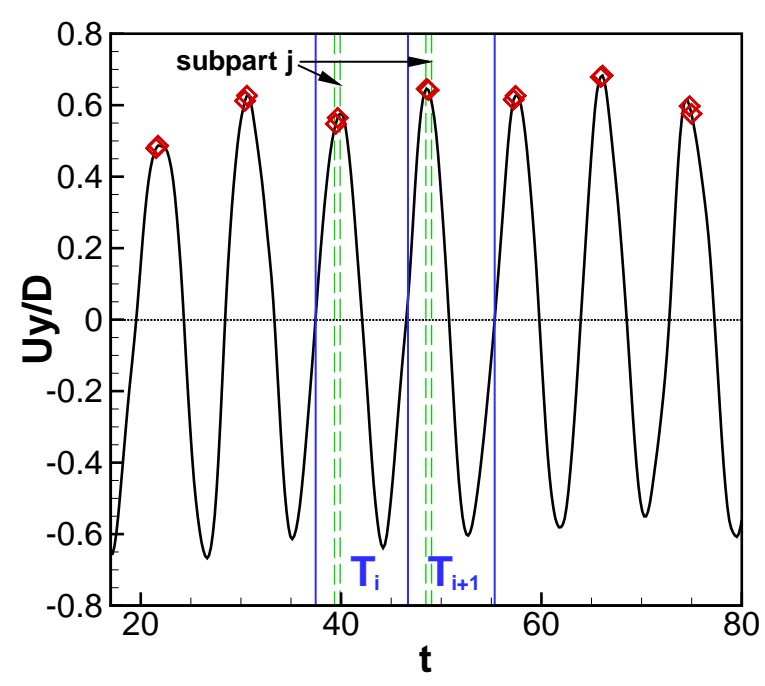

(b) The selected points in red found in a period subpart in green will be averaged to give the reference position of the corresponding period sub-part.

Figure 10: A representative signal of the present FSI phenomenon: the time history of the ydisplacements $U_{y}$ of the shell extremity.

\subsection{Application of the method}

The simulation data containing structure positions, pressure and velocity fields, are generated every 150 time steps. According to the frequency observed for the structure and the timestep size chosen about 50 data sets are obtained per swiveling period. With respect to the time interval predicted and the number of subparts chosen, the data for each subpart are averaged from about 50 data sets. A post-processing program is implemented based on the method described above. It does not require any special treatment and thus the aforementioned method to get the phase-resolved results is straightforward.

For the experiments different ways to apply the phase-averaging technique can be found: 
Gomes and Lienhart (2006) used a FPGA (Field Programmable Gate Array) with a $1 \mathrm{MHz}$ internal clock to monitor two main events in parallel during the acquisition: On the one hand the PIV measurements and on the other hand the beginning of each structure motion cycle. After obtaining all the data a post-processing software sorted and reconstructed the phaseaveraged results based on the correlated information given by the FPGA. It is possible to implement such an analysis method if the beginning of each structure motion cycle can easily be detected. In Gomes and Lienhart (2006) the starting position of the swiveling period was determined in real time using an electronic angular position sensor. Owing to the fixed cylinder such a signal is not available in the present configuration.

Thus, the current experimental setup uses a similar, but less complex reconstruction method. It consists of the multiple-point triangulation sensor described in Section 3.2 and the synchronizer of the PIV system. Each measurement pulse of the PIV system is detected in the data acquisition of the laser distance sensor, which measures the structure deflection continuously with 800 profiles per second. With this setup, contrary to Gomes and Lienhart (2006), the periods are not detected during the acquisition but in the post-processing phase. After the run a specific software based on the described method mentioned above computes the reference structure motion period and sorts the PIV data to get the phase-averaged results.

\section{Unsteady Results}

In order to comprehend the real structure deformation and the turbulent flow field found in the present test case, experimentally and numerically obtained unsteady results are presented in this section.

Figure 11 shows experimental raw signals of dimensionless displacements from a point located at a distance of $9 \mathrm{~mm}$ from the shell extremity in the midplane of the test section. In Figure 11(a) the history of the y-displacement $U_{y}^{*}=U_{y} / D$ obtained in the experiment is plotted. The signal shows significant variations in the extrema: The maxima of $U_{y}^{*}$ vary between 0.298 and 0.523 and the minima between -0.234 and -0.542 . The standard deviations on the extrema are about \pm 0.05 ( $\pm 12 \%$ of the mean value of the extrema). Minor variations are observed regarding the period in Figure 11(a). Figure 11(b) and 11(c) show the corresponding experimental phase portrait and phase plane, respectively. The phase portrait has a quasi-ellipsoidal form. The monitoring point trajectory plotted in the phase plane describes an inversed "C", which is typical for the first swiveling mode. The cycle-to-cycle variations in these plots are small. Therefore, the FSI phenomenon can be characterized as quasi-periodic.

Figure 12 is composed of eight images of the instantaneous flow field (streamwise velocity component) experimentally measured in the $\mathrm{x}-\mathrm{y}$ plane located in the middle of the rubber plate. These pictures constitute a full period $T$ of the FSI phenomenon arbitrarily chosen. As mentioned before, the shell deforms in the first swiveling mode. Thus, there is only one wave node located at the clamping of the flexible structure. At the beginning of the period $(t=0)$ the structure is in its undeformed state. Then, it starts to deform upwards and reaches a maximal deflection at $t \approx T / 4$. Afterwards, the shell deflects downwards until its maximal deformation at $t \approx 3 T / 4$. Finally the plate deforms back to its original undeformed state and the end of the period is reached. It should be pointed out that very similar figures as depicted in Fig. 12 could also be shown from the numerical predictions based on LES. Exemplary and for the sake of brevity, Fig. 13 displays the streamwise velocity component of the flow field in a x-y-plane only at $t \approx 3 T / 4$.

As visible in Fig. 12 and in Fig. 13 the flow is highly turbulent, particularly near the cylinder, the flexible structure and in the wake. As expected the LES prediction is capable to resolve 


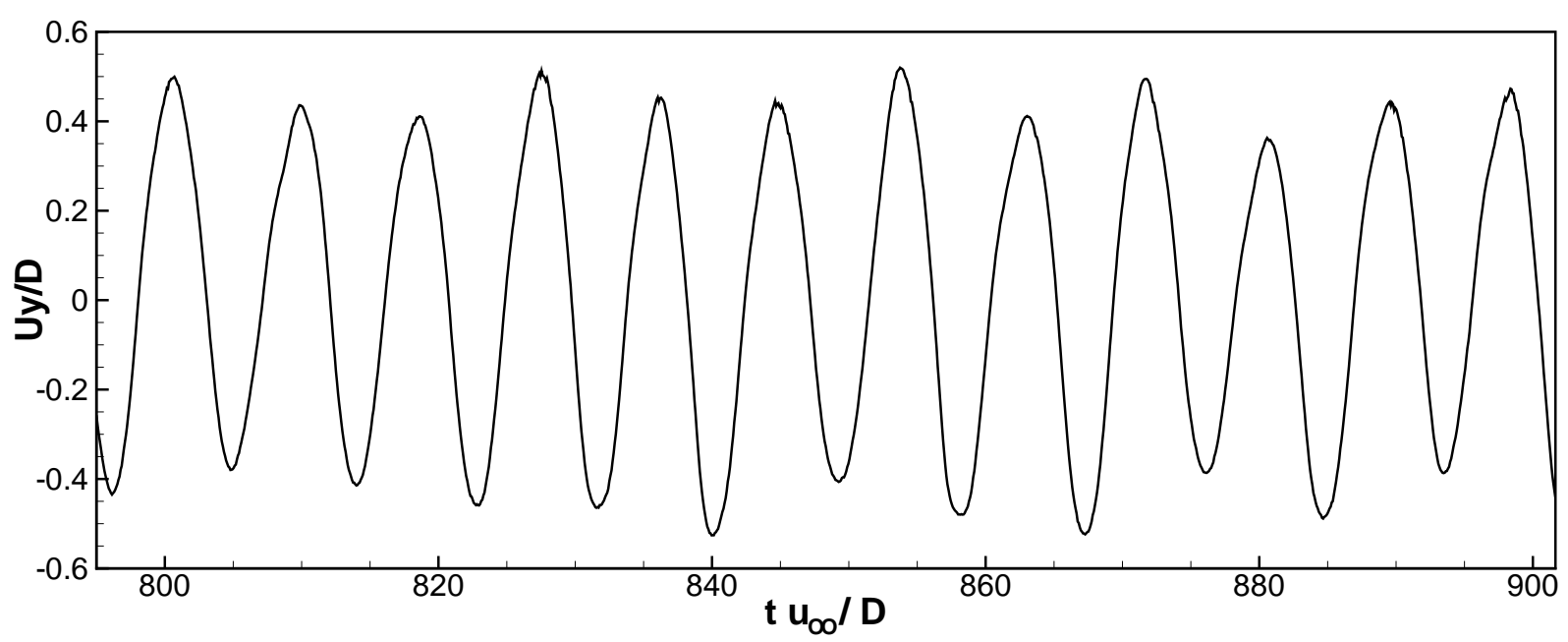

(a) y-displacement vs. time

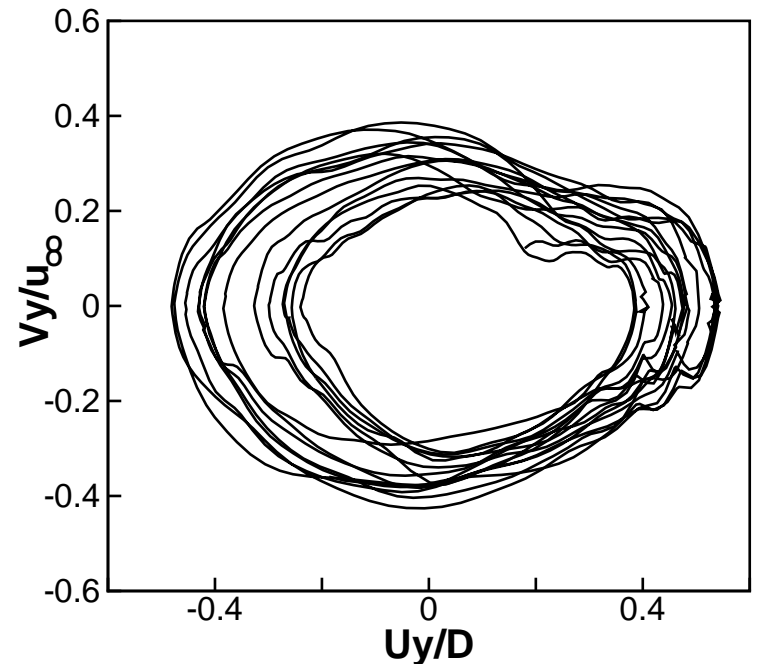

(b) Phase portrait: y-velocity vs. y-displacement

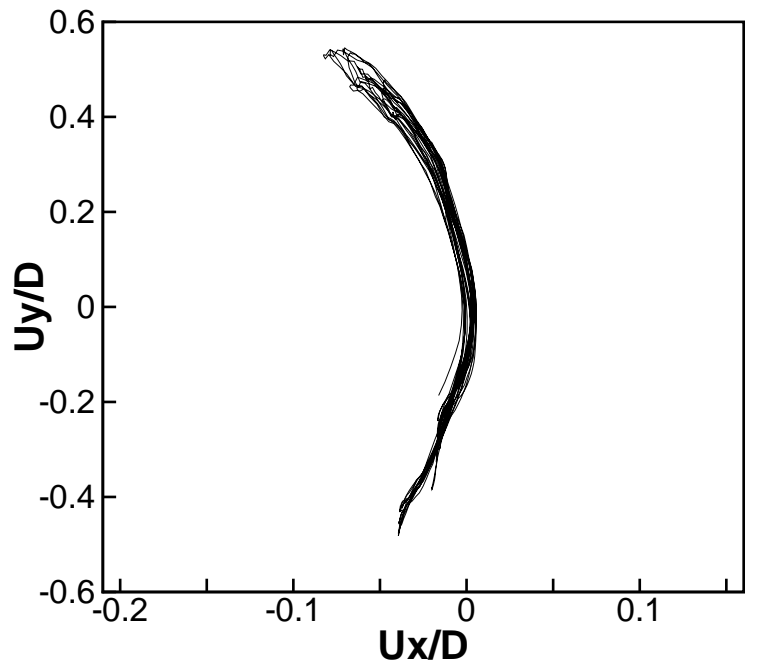

(c) Phase plane: y-displacement vs. $\mathrm{x}$-displacement

Figure 11: Experimental raw signals of dimensionless displacements from a point in the midplane of the test section located at a distance of $9 \mathrm{~mm}$ from the shell extremity.

small-scale flow structures in the wake region and in the shear layers. The strong shear layers originating from the separated boundary layers are clearly visible. This is the region where for the sub-critical flow the transition to turbulence takes place as visible in the figures. Consequently, the flow in the wake region behind the cylinder is obviously turbulent and shows cycle-to-cycle variations. That means the flow field in the next periods succeeding the interval depicted in Fig. 12 will definitely look slightly different due to the irregular chaotic character of turbulence. Therefore, in order to be able to compare these results an averaging method is needed leading to a statistically averaged representation of the flow field. Since the FSI phenomenon is quasi-periodic the phase-averaging procedure presented above is ideal for this purpose and the results obtained are presented in the next section.

\section{Phase-resolved Results and Discussion}

The following part is divided into two different sections: in the first one numerical phasedresolved results obtained for the two configurations (full and subset case) are compared. Based 


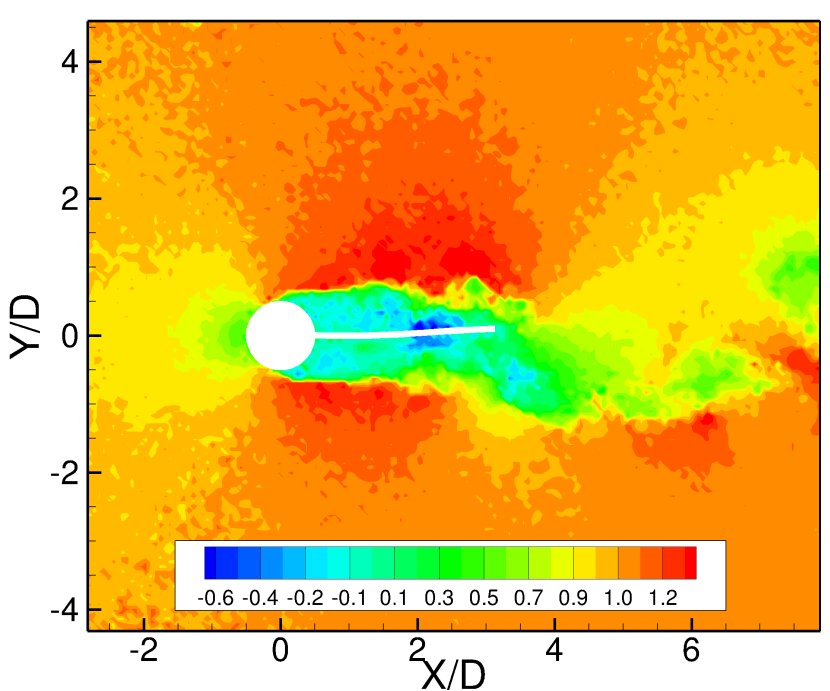

(a) $t \approx T / 8$

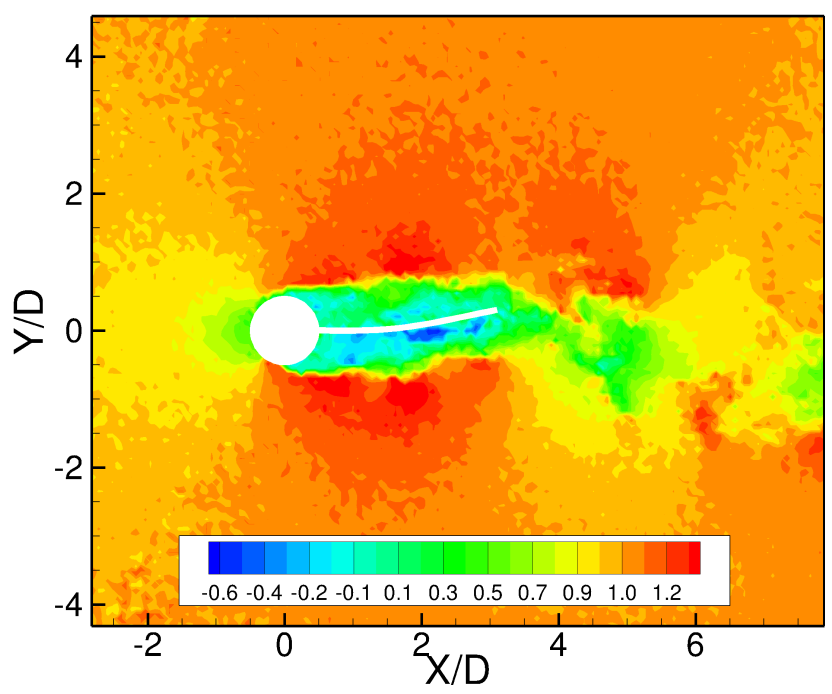

(c) $t \approx 3 T / 8$

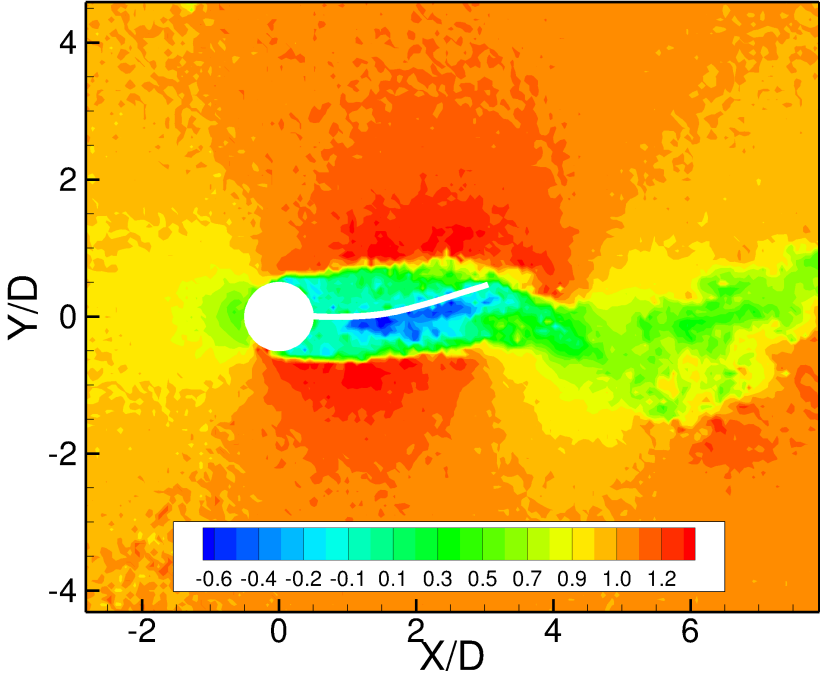

(b) $t \approx T / 4$

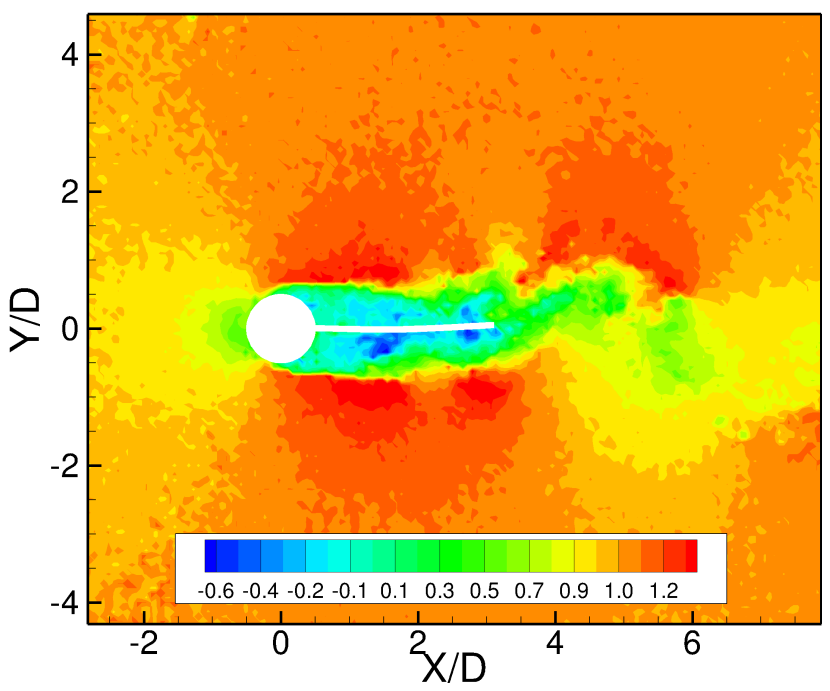

(d) $t \approx T / 2$

Figure 12: Experimental unsteady flow field for $t \in] 0, T / 2]$ ( $\mathrm{x}-\mathrm{y}$ plane located in the middle of the rubber plate).

on this evaluation one case is chosen for a parameter study. Then, in the second subsection the numerical phased-averaged results chosen are juxtaposed to the experimental ones in order to verify their quality.

In both simulations (subset and full case) the flow is initialized by assuming the entire structure to be non-deformable. In this case the shell attached to the backside of the cylinder acts like a splitter plate attenuating the generation of a von Kàrmàn vortex street behind the cylinder. Nevertheless, quasi-periodic vortex shedding is still observed with a Strouhal number of $\mathrm{St}_{\text {fixed }} \approx 0.175$. Owing to different loads on both sides the structure starts to deflect as soon as it is released. After a short initial phase, in which the amplitudes of the deflections successively increase, a new quasi-periodic mode of oscillation is reached. In accordance with the experiment in the numerical simulations the shell deforms in the first swiveling mode as visible in Fig. 14. 


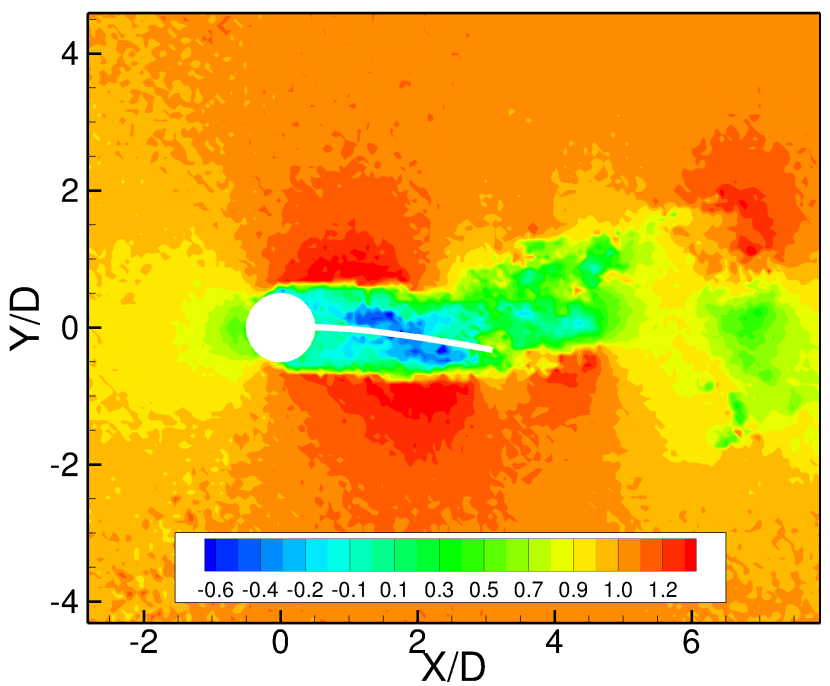

(e) $t \approx 5 T / 8$

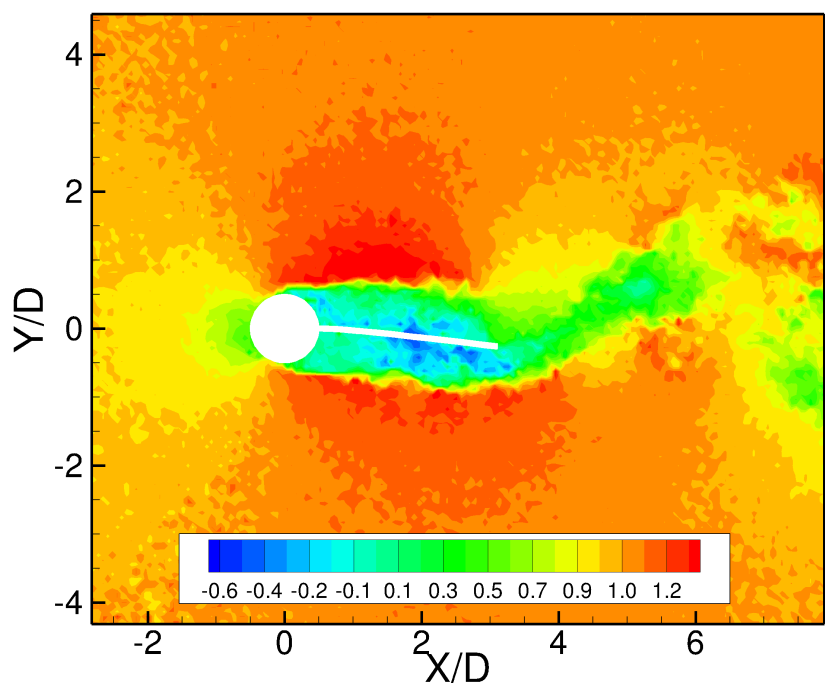

(g) $t \approx 7 T / 8$

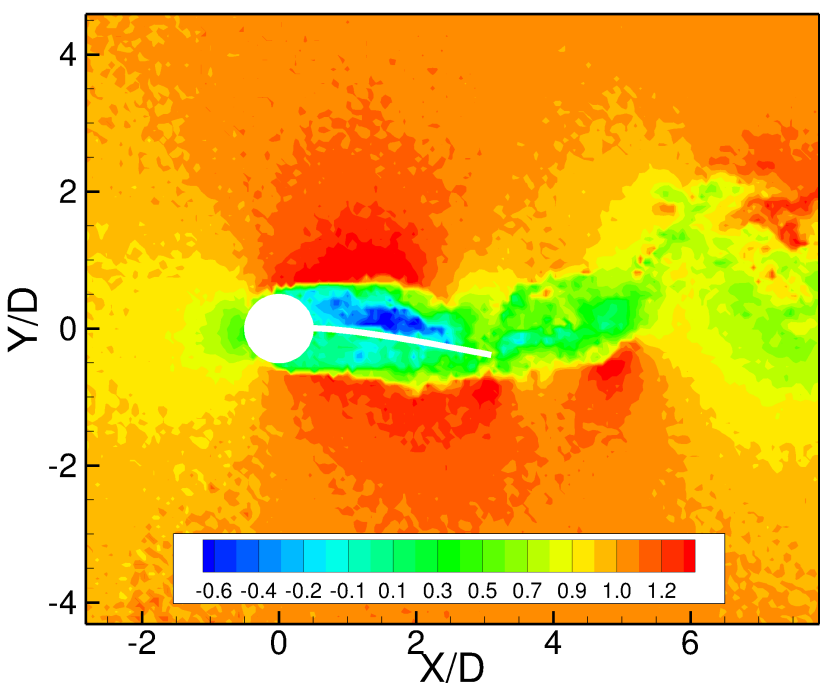

(f) $t \approx 3 T / 4$

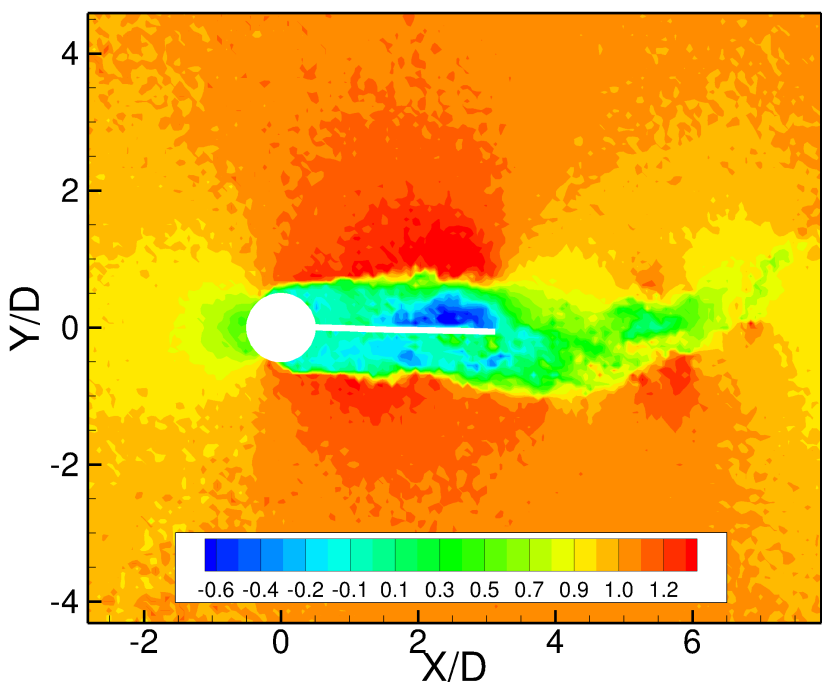

(h) $t \approx T$

Figure 12: (continued): Experimental unsteady flow field for $t \in] T / 2, T]$ (x-y plane located in the middle of the rubber plate).

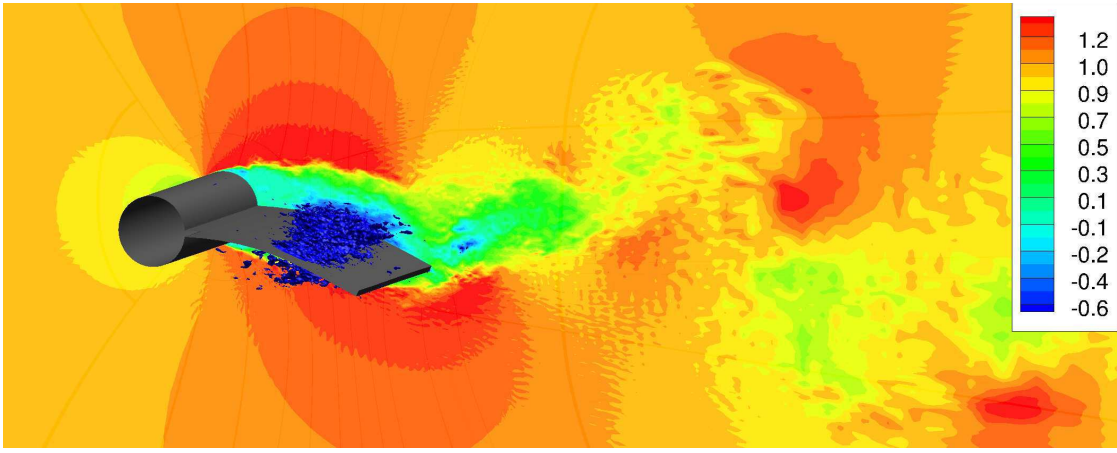

(a) Strong backflow region $u / u_{\text {inflow }}<-0.6$ visualized by blue iso-surface.

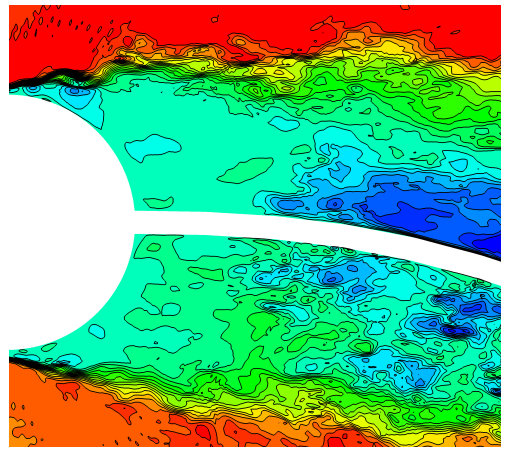

(b) Zoom of the shear layers.

Figure 13: Unsteady flow field and deformed structure predicted by LES at $t \approx 3 T / 4$. 


\subsection{Comparison of numerical results}

Two numerical setups are used to run the FSI-PfS-1a simulation: the full case and the subset case. These configurations differ regarding the geometry and the boundary conditions as described in Section 4.4. The subset case represents a simpler model than the full case requiring less CPU-time (one second real-time is predicted in about 170 hours wall-clock with the subset case on 84 processors and in about 310 hours wall-clock with the full case on 142 processors). Similar savings can be achieved with respect to the memory requirements of both cases. The full case requires a maximum of 231 Mbytes per core and about 32 Gbytes for all processors. The subset case needs 242 Mbytes per core, which leads to about 20 Gbytes for the entire simulation. Thus the subset is worth to be considered. The question, however, is which influence these modeling assumptions have on the numerical results?

\subsubsection{Full case vs. subset case}

Both setups are performed with slightly different material characteristics than defined in Section 2.4: The Young's modulus is set to $E=14 \mathrm{MPa}$, the thickness of the rubber plate is equal to $h=0.002 \mathrm{~m}$, the solid density is $\rho_{\text {rubber plate }}=1425 \mathrm{~kg} \mathrm{~m}^{-3}$ and no structural damping is used. The reason is that this comparison was a preliminary study carried out prior to the final definition of the test case. Because of the similitude of the values used here and those defined in Section 2.4 and because of the large CPU-time requested, the comparison of the numerical results is not repeated with the parameters defined in Section 2.4 .

\section{Deflection of the structure.}

At first the predicted deformation of the structure is analyzed. For this purpose Fig. 14 depicts an arbitrarily chosen snapshot of the deformed structure for both cases taken from the quasiperiodic oscillation mode. It is observed that the flexible structure in the full case deforms more strongly in z-direction than in the subset case. This observation can be explained as follows: the full setup has a wider structure and the lateral nodes are exposed to less constraints than in the subset case.

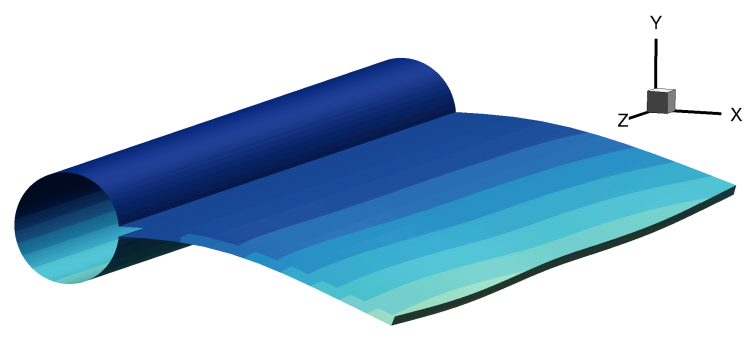

(a) Full case (small z-deformations of the structure)
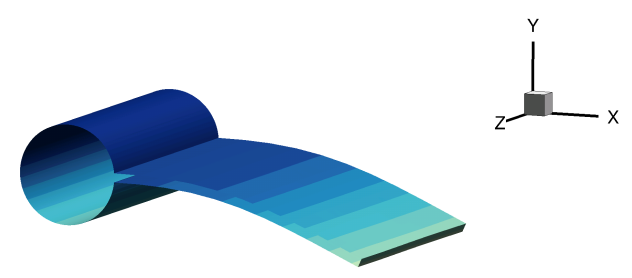

(b) Subset case (nearly no z-deformations of the structure)

Figure 14: Comparison of the structure deformations in y- and z-direction between the full and subset case.

In order to quantify these displacement variations along the z-axis in the full case, three characteristic points on the structure in three parallel planes depicted in Fig. 15(c) are chosen: One plane is set in the middle of the structure, the others are shifted $\pm 60 \mathrm{~mm}$ in the spanwise direction. All three points are not located directly on the shell extremity but at a distance of 9 $\mathrm{mm}$ from the extremity. This choice is motivated by the planned comparison with the measured data (Section 7.2) and the limitation in the experiment. The laser distance sensor does not 
allow to follow the structure extremity and thus points at a certain distance from the tail are chosen. The dimensionless y-displacements $U_{y}^{*}=U_{y} / D$ at these three points are monitored as shown in Fig. 15(a). The following observation can be made: 1. The displacements are in phase. 2. Local differences between the curves are observed in the extrema. 3. These variations are, however, not constant in time. In other words the displacement in one plane is not always bigger than another. The variations reflect some kind of waves in the structure that move in the spanwise direction. Comparing those three raw signals with the z-averaged displacements depicted in Fig. 15(b), a maximal difference of 5\% regarding the extrema is noticed. Hence the variations are small. The corresponding z-variations of the subset case are even smaller $(<0.5 \%)$. Therefore, it was decided to continue the analysis by averaging both cases in z-direction. Notice that by the averaging procedure in z-direction the 3D-problem is reduced to a $2 \mathrm{D}$-problem.

The next step is to compare the structure deformations obtained with the full and the subset case. Figure 15(b) shows the z-averaged dimensionless y-displacements of both cases taken at $9 \mathrm{~mm}$ from the extremity. The frequencies are identically predicted in both cases $\left(f_{\mathrm{FSI}_{\mathrm{num}}}=6.96 \mathrm{~Hz}\right.$ and $\left.\mathrm{St}_{\mathrm{FSI}_{\mathrm{num}}}=0.11\right)$. Minor differences appear in the extrema of the raw signals presented in Fig. 15(b). As before these variations are not constant in time and thus the maximal values are found irregularly for either the full or the subset case. As a consequence the comparison of the phase-averaged displacement signal (see Fig. 15(d)) shows no significant changes between both cases and the coefficient of determination $R^{2}=$ $1-\sum_{i}\left(U_{y_{i}}^{*}-\hat{U}_{y_{i}}^{*}\right)^{2} / \sum_{i}\left(U_{y_{i}}^{*}-\overline{U_{y}^{*}}\right)^{2}$ of the calculated mean phase is close to unity $(0.9869$ for the full case and 0.9782 for the subset case). $\hat{U}_{y_{i}}^{*}$ denotes the estimated mean value of $U_{y}^{*}$ for the point i. $\overline{U_{y}^{*}}$ is the mean value of all the displacements. The standard deviation for each point of the averaged phase is also computed: the maximum for the full case is 0.055 (dimensionless) and for the subset case 0.065 (dimensionless). These values are small compared to the signal, which is another indication for the reliability of the averaged phase. The subset case predicts structure deformations very similar to the full case. In order to check if the FSI results are quasi identical for the full and the subset case, the phase-resolved flow field has to be additionally taken into account.

\section{Phase-resolved flow field.}

The phase-averaging process described in Section 5 delivers the phase-resolved flow fields for the full and the subset case. In order to compare both just two representative phase-averaged positions of the FSI problem are chosen to limit this subsection. Figure 16 shows the flow field in the vicinity of the shell during its maximal deformation at $t \approx T / 4$ and Fig. 17 depicts it close to its undeformed position at $t \approx T$, where $T$ denotes the period time of the phase-averaged signal. The figures display the contours of the phase-averaged streamwise and transverse velocity components. Furthermore, the local error of the velocity magnitude defined by the deviation between the absolute values of the velocity vector of both cases normalized by the inflow velocity $u_{\text {inflow }}$ is depicted. For both positions the results obtained for the subset and full case are nearly identical. Figures 16(e) and 17(e) underline that the local error of the velocity magnitude between both cases is about zero everywhere except in the region near the structure. For the position $t \approx T / 4$ (Fig. 16(e)) small local errors are located behind the structure in the vortex shedding region. For the position $t \approx T$ (Fig. 17(e)) the phase-averaged position of the shell for the subset case differs slightly from the one of the full case. Since the flow field is rapidly changing during the vortex shedding process, this minor deviation in the phase-angle explains the small local errors observed near the structure and in the shear layer. The comparison of the phase-averaged flow fields shows no significant changes between both 


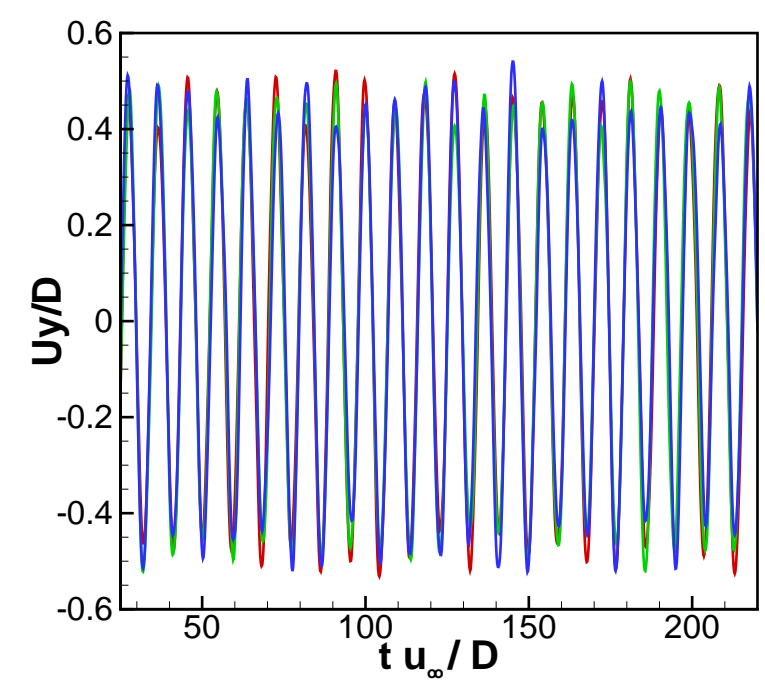

(a) Comparison of the numerical raw dimensionless displacements at the three points sketched in Fig. 15(c) (full case).

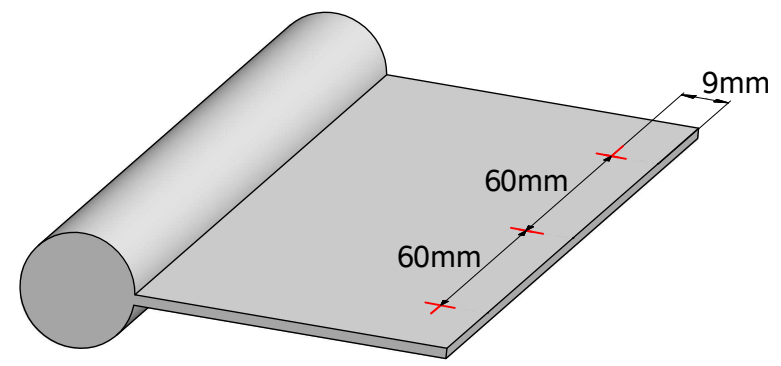

(c) Position of the monitoring points (in red) for the full case.

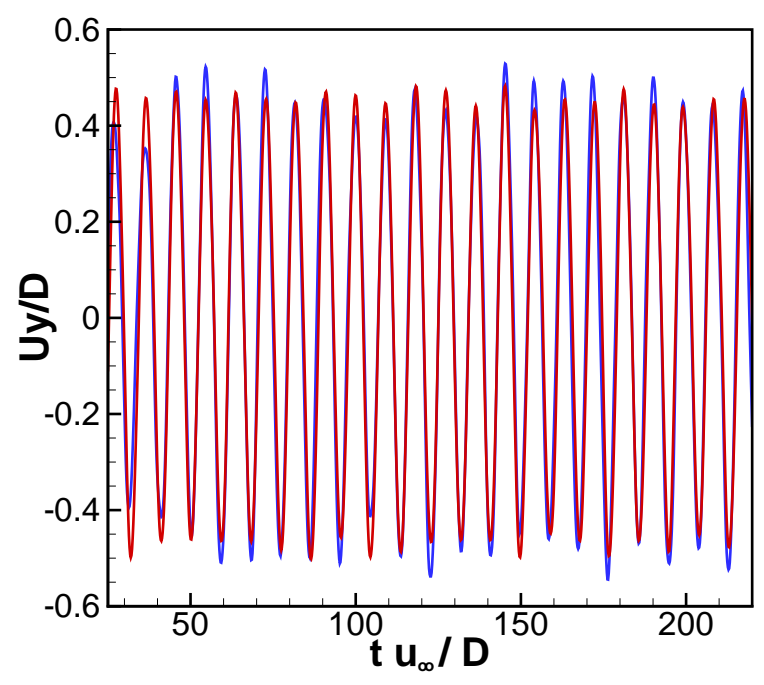

(b) Comparison of the numerical z-averaged dimensionless displacements between the full (red) and the subset (blue) case.

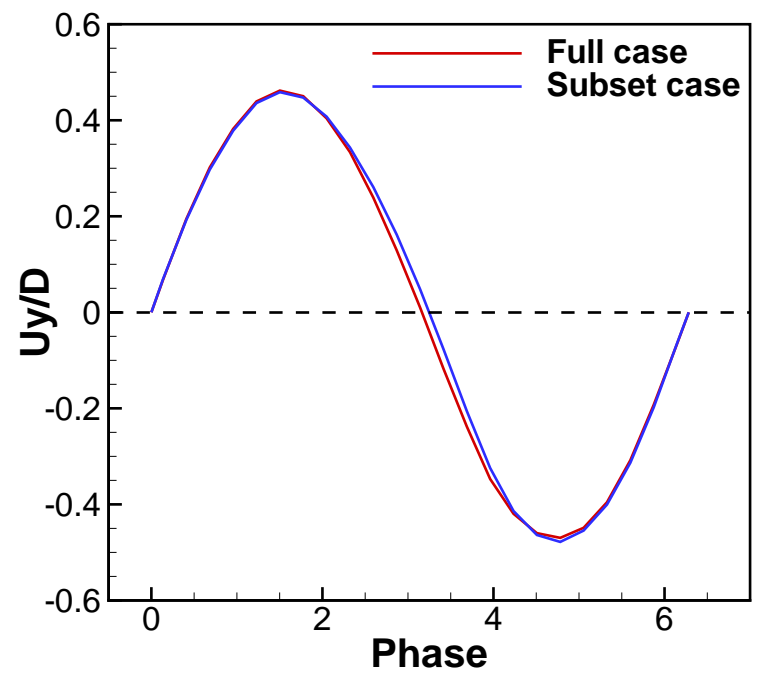

(d) Comparison of the averaged phase of the full and the subset case.

Figure 15: Comparison of numerical results for the full case and the subset case.

cases. The subset case predicts the phase-averaged flow field very similar to the full case. As said before, the subset setup is simpler and less expensive in CPU-time. Therefore, the subset case is very interesting in order to simulate the present test case using LES.

\subsubsection{Sensitivity study for the subset case - Dimensional analysis}

In order to better understand the test case a dimensional analysis was carried out. The physical quantities of the present FSI problem are: The dynamic viscosity $\mu_{f}$, the fluid density $\rho_{f}$, the inlet velocity $u_{\text {inflow }}$ for the fluid; the cylinder diameter $D$, the dimensions of the rubber plate $l, w$ and $h$; the Young's modulus $E$, the Poisson's ratio $\nu$ and the density of the rubber plate $\rho_{\text {rubber plate; }}$ To describe the FSI phenomenon the FSI frequency $f_{\mathrm{FSI}}$, the displacement extrema $\left.U_{y}\right|_{\max }$ and $\left.U_{y}\right|_{\min }$ are chosen. These 13 physical quantities lead to 10 dimensionless parameters: The Reynolds number $\operatorname{Re}=\rho_{f} u_{\text {inflow }} D / \mu_{f}$ for the fluid; the length ratios $w / l, h / l$, $D / l$ for the geometry; $\nu$ for the material of the rubber plate; The density ratio $\rho_{f} / \rho_{\text {rubber }}$ plate, 
the Cauchy number $\mathrm{Cy}=\rho_{f} u_{\text {inflow }}^{2} / E$ (as defined in De Langre (2002)), the extrema of the dimensionless y-displacements $\left.U_{y}^{*}\right|_{\max }=\left.U_{y}\right|_{\max } / D$ and $\left.U_{y}^{*}\right|_{\min }=\left.U_{y}\right|_{\min } / D$ and the Strouhal number $\mathrm{St}_{\mathrm{FSI}}=f_{\mathrm{FSI}} D / u_{\text {inflow }}$ for the FSI coupling.

In the present experimental investigation the operating conditions for the fluid are well-known. The length and the width of the rubber plate are well defined, too. Therefore, the Reynolds number Re, the geometrical ratios $w / l$ and $D / l$ are fixed in the sensitivity study.

- On the contrary, as mentioned in Section 2.1, the material is natural rubber and to manufacture a perfectly homogeneous $2 \mathrm{~mm}$ plate is not easy. The experimental measurements show that the thickness varies between 0.002 and $0.0022 \mathrm{~m}$. Two values of $h$ are tested: the theoretical value of $0.002 \mathrm{~m}$ and the average value $0.0021 \mathrm{~m}$. Consequently, the geometrical ratio $h / l$ will be taken into account in the sensitivity study.

- The density of the rubber plate $\rho_{\text {rubber plate }}$ is determined by a scale and the volume of the structure. Due to the dependency of this volume on the plate thickness the density determination can be inaccurate. As an additional dimensionless parameter the density ratio $\rho_{f} / \rho_{\text {rubber plate }}$ is a part of the sensitivity study.

- The last parameter of the structure is the Young's modulus, because it has an important influence on the modeling of the material. A range of values for $E$ and consequently of $\mathrm{Cy}$ is tested to evaluate this influence.

The dimensional analysis presented here will also be reduced to the six following dimensionless

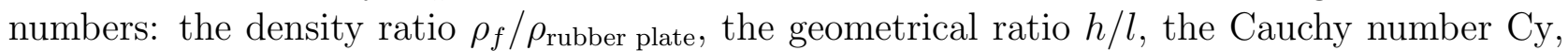
the dimensionless y-displacement extrema $\left.U_{y}^{*}\right|_{\max }$ and $\left.U_{y}^{*}\right|_{\min }$ and the Strouhal number $\mathrm{St}_{\mathrm{FSI}}$.

All the tests were carried out without structural damping and are summarized in Table 4. The full case used in Section 7.1.1 and the experimental results are also added as references. Each simulation was done for a time interval of $4 \mathrm{~s}$ physical time and comprises about 27 swiveling periods. Relative errors between the numerical and experimental values are given.

The following results and trends can be seen:

- By varying the Young's modulus $E$ between 8 and $16 \mathrm{MPa}\left(240 \times 10^{-6} \geq \mathrm{Cy} \geq 120 \times 10^{-6}\right)$ it is possible to control the mode of the FSI phenomenon. Thus $E$ (or the Cauchy number) turns out to be the most crucial material parameter. With $E$ smaller than $9 \mathrm{MPa}(\mathrm{Cy}$ $\geq 213 \times 10^{-6}$ ), the system oscillates in the second swiveling mode (similar to the second bending mode presented in Fig. B.25). With $E$ larger than $12 \mathrm{MPa}\left(\mathrm{Cy} \leq 160 \times 10^{-6}\right)$ the structure deflection is dominated by the first bending mode of the structure. For a Young's modulus between 9 and $12 \mathrm{MPa}\left(213 \times 10^{-6} \geq \mathrm{Cy} \geq 160 \times 10^{-6}\right)$ a mode transition phase appears in which both swiveling modes are apparent. In this situation the ydisplacements of the flexible structure extremity are no longer quasi-periodic and can not be described by a unique frequency.

- Non-negligible variations in the density $\left(1320 \mathrm{~kg} \mathrm{~m}^{-3} \leq \rho_{\text {rubber plate }} \leq 1725 \mathrm{~kg} \mathrm{~m}^{-3}\right)$ $\left(0.757 \geq \rho_{f} / \rho_{\text {rubber plate }} \geq 0.580\right)$ for a fixed thickness $(\mathrm{h}=0.002 \mathrm{~m})$ and Young's modulus $(E=14 \mathrm{MPa})$ do not drastically change the results of the frequency and of the mean period extrema. The FSI frequency $f_{\mathrm{FSI}}$ (respectively the Strouhal number $\mathrm{St}_{\mathrm{FSI}}$ ) slightly decreases with the increase of the density. 


\begin{tabular}{|c|c|c|c|c|c|c|c|c|c|c|c|c|c|c|}
\hline \multirow[t]{2}{*}{ Case } & \multicolumn{3}{|c|}{ Materials parameters } & \multicolumn{3}{|c|}{ Dimensionless numbers } & \multicolumn{8}{|c|}{ Results } \\
\hline & \begin{tabular}{|c|} 
Thickness \\
$\mathrm{h}(\mathrm{mm})$
\end{tabular} & $\begin{array}{c}\text { Density } \\
\left(\mathrm{kg} \mathrm{m}^{-3}\right) \\
\end{array}$ & $\begin{array}{c}\mathrm{E} \\
(\mathrm{MPa})\end{array}$ & $\mathrm{h} / \mathrm{l}$ & $\frac{\rho_{f}}{\rho_{\text {rubber plate }}}$ & $\mathrm{Cy}$ & $\begin{array}{l}f_{\mathrm{FSI}} \\
(\mathrm{Hz}) \\
\end{array}$ & $\mathrm{St}_{\mathrm{FSI}}$ & \begin{tabular}{|c|} 
Error \\
$(\%)$
\end{tabular} & $\left|U_{y}^{*}\right|_{\max }$ & $\begin{array}{c}\text { Error } \\
(\%) \\
\end{array}$ & $\left.U_{y}^{*}\right|_{\min }$ & \begin{tabular}{|c|} 
Error \\
$(\%)$
\end{tabular} & Comments \\
\hline \multirow{16}{*}{ Subset case } & 2 & 1320 & 14 & 0.033 & 0.757 & $137 \times 10^{-6}$ & 7.00 & 0.1112 & -1.40 & 0.460 & 10.1 & $\mid-0.488$ & -16.4 & first swiveling mode \\
\hline & 2 & 1425 & 14 & 0.033 & 0.702 & $137 \times 10^{-6}$ & 6.96 & 0.1106 & -2.02 & 0.463 & 10.6 & -0.457 & -8.9 & first swiveling mode \\
\hline & 2 & 1725 & 14 & 0.033 & 0.580 & $137 \times 10^{-6}$ & 6.94 & 0.1102 & -2.22 & 0.458 & 9.6 & -0.478 & -13.9 & first swiveling mode \\
\hline & 2 & 1425 & 8 & 0.033 & 0.702 & $240 \times 10^{-6}$ & - & - & - & - & - & - & - & second swiveling mode \\
\hline & 2 & 1425 & 9 & 0.033 & 0.702 & $213 \times 10^{-6}$ & - & - & - & - & - & - & - & second mode+transition \\
\hline & 2 & 1425 & 10 & 0.033 & 0.702 & $192 \times 10^{-6}$ & - & - & - & - & - & - & - & second mode+transition \\
\hline & 2 & 1425 & 11 & 0.033 & 0.702 & $174 \times 10^{-6}$ & - & - & - & - & - & - & - & transition \\
\hline & 2 & 1425 & 12 & 0.033 & 0.702 & $160 \times 10^{-6}$ & - & - & - & - & - & - & - & transition+first mode \\
\hline & 2 & 1425 & 14 & 0.033 & 0.702 & $137 \times 10^{-6}$ & 6.96 & 0.1106 & -2.02 & 0.463 & 10.6 & -0.457 & -8.9 & first swiveling mode \\
\hline & 2 & 1425 & 15 & 0.033 & 0.702 & $129 \times 10^{-6}$ & 7.04 & 0.1118 & -0.84 & 0.448 & 7.2 & -0.481 & -14.6 & first swiveling mode \\
\hline & 2 & 1425 & 16 & 0.033 & 0.702 & $120 \times 10^{-6}$ & 7.10 & 0.1128 & -0.01 & 0.471 & 12.6 & -0.473 & -12.7 & first swiveling mode \\
\hline & 2.1 & 1360 & 14 & 0.035 & 0.735 & $137 \times 10^{-6}$ & 7.00 & 0.1112 & -1.38 & 0.463 & 10.6 & -0.471 & -12.2 & first swiveling mode \\
\hline & 2.1 & 1360 & 15 & 0.035 & 0.735 & $129 \times 10^{-6}$ & 7.07 & 0.1123 & -0.50 & 0.461 & 10.3 & -0.479 & -14.0 & first swiveling mode \\
\hline & 2.1 & 1360 & 16 & 0.035 & 0.735 & $120 \times 10^{-6}$ & 7.08 & 0.1125 & -0.25 & 0.456 & 9.1 & -0.464 & -10.6 & first swiveling mode \\
\hline & 2.1 & 1360 & 18 & 0.035 & 0.735 & $107 \times 10^{-6}$ & 7.13 & 0.1133 & 0.36 & 0.447 & 6.8 & -0.462 & -10.2 & first swiveling mode \\
\hline & 2.1 & 1360 & 20 & 0.035 & 0.735 & $96 \times 10^{-6}$ & 7.20 & 0.1144 & 1.32 & 0.452 & 8.0 & -0.461 & -9.7 & first swiveling mode \\
\hline Full case & 2 & 1425 & 14 & 0.033 & 0.702 & $137 \times 10^{-6}$ & 6.96 & 0.1106 & -2.02 & 0.465 & 11.2 & -0.473 & -12.6 & first swiveling mode \\
\hline Experiments & & & & & & & 7.10 & 0.1128 & - & 0.418 & - & -0.420 & - & first swiveling mode \\
\hline
\end{tabular}

Table 4: Parameter study for the subset case of the FSI test case (without structural damping). 
- Comparing the results for both thicknesses for the range $14 \mathrm{MPa} \leq E \leq 16 \mathrm{MPa}$, it is obvious that a mild variation of the thickness of the rubber plate $(0.1 \mathrm{~mm}$, equivalent to $5 \%$ ) has a non-negligible influence on the extrema of the mean period and no significant influence on the frequency.

- Overall the frequency of the FSI phenomenon $f_{\mathrm{FSI}}$ (respectively the Strouhal number $\mathrm{St}_{\mathrm{FSI}}$ ) is very well predicted (relative error under $2.22 \%$ ) for all tested parameters leading to the first swiveling mode.

- Comparing the results for the density $\rho_{\text {rubber plate }}=1360 \mathrm{~kg} \mathrm{~m}^{-3}$ in the range $14 \mathrm{MPa} \leq$ $E \leq 20 \mathrm{MPa}$, we observe that the FSI frequency $f_{\mathrm{FSI}}$ (respectively the Strouhal number $\left.\mathrm{St}_{\mathrm{FSI}}\right)$ slightly increases with the Young's modulus and that the displacement extrema decrease.

In summary, the parameter study shows that the Young's modulus (or the dimensionless number of Cauchy $\mathrm{Cy}$ ) is the most important parameter: It controls the swiveling mode of the flexible structure. Furthermore, it can be observed that mild modifications of the shell thickness (or of the geometrical ratio $h / l$ ) have a certain effect on the predicted FSI phenomenon. Contrarily, this parameter study shows that variations of the density ratio do not have major influence on the predictions. Therefore, errors in the density measurement do not play an important role. With the support of these extensive preliminary numerical investigations we can now compare the final numerical results with the experiment.

\subsection{Comparison between numerical and experimental results}

The investigations presented in Section 7.1.1 based on slightly different material characteristics than defined in Section 2.4 have shown that the subset case permits a gain in CPU-time but nevertheless nearly identical results as the full case. Therefore, the numerical computation with the structural parameters defined in Section $2.4\left(E=16 \mathrm{MPa}, h=0.0021 \mathrm{~m}, \rho_{\text {rubber plate }}\right.$ $=1360 \mathrm{~kg} \mathrm{~m}^{-3}$ ) is carried out for the subset case.

Two simulations are considered: One with the structural damping defined in Appendix B, the other one without damping. These results are compared with the experimental data to check their accuracy. In order to quantitatively compare the experimental and numerical data, both are phase-averaged as explained in Section 5. Similar to the numerical comparison presented in Section 7.1.1 the displacement of the structure will be first analyzed and then the phaseresolved flow field is considered.

\subsubsection{Deflection of the structure}

The structure contour of the phased-averaged experimental results for the reference period is depicted in Fig. 18. Obviously, the diagram represents the first swiveling mode of the FSI phenomenon showing only one wave mode at the clamping. Figure 19(a) depicts the experimental dimensionless raw signal obtained at a point located in the midplane at a distance of $9 \mathrm{~mm}$ from the shell extremity. Figure 19(b) shows the numerical signal predicted without structural damping and Fig. 19(c) the one computed with damping. Applying the phase-averaging process the mean phase of the FSI phenomenon for the experiment and for the simulations is generated. The outcome is presented in Fig. 19(d) with the phase as the abscissa and the dimensionless displacement $U_{y}^{*}=U_{y} / D$ as the ordinate. The amplitudes of the experimental signal varies more than in the predictions. Therefore, the maximal standard deviation of each point of the averaged phase is for the experiment bigger (0.083) than for the simulation (0.072 with and without damping). In order to check the reliability of the computed mean phase 
the coefficient of determination $R^{2}$ is computed: it is smaller for the mean experimental phase (0.9640) than for the mean simulation ones (0.9770 without damping and 0.9664 with damping). However, the values are close to unity, which is an indication that the averaged phases are representative for the signals. In Fig. 19(d) the mean period calculated from the simulation without damping is quasi-antisymmetric with respect to $U_{y}^{*}=0$. On the contrary the period derived from the experiment is not exactly antisymmetric with respect to the midpoint of the phase $\phi=\pi$ : The cross-over is not at the midpoint of the phase but slightly shifted to the right. However, the absolute values of the minimum and maximum are nearly identical. As for the experimental phase, the simulation with damping generates a phase signal, which is not completely antisymmetric. In the experiment this weak asymmetry can be attributed to minor asymmetries in the setup or in the rubber material. The comparison in Fig. 19(d) shows some differences in the extrema and a summary is presented in Table 5. Without structural damping the simulations produce extrema which are too large by about $10 \%$. With structural damping the extrema are smaller, even smaller than in the experiment by about $4 \%$. Thus, the structural damping also has a significant influence on the FSI predictions and can not be overlooked.

The frequency of the FSI phenomenon, i.e., the frequency of the y-displacements, is about $f_{\mathrm{FSI}}$ exp $=7.10 \mathrm{~Hz}$ in the experimental investigations, which corresponds to a Strouhal number $\mathrm{St}_{\mathrm{FSI}} \approx 0.11$. In the numerical predictions without damping this frequency is $f_{\mathrm{FSI}}^{\text {no dam }}$ ing $=7.08 \mathrm{~Hz}$ and with damping $f_{\mathrm{FSI} \text { num }}^{\text {damping }}=7.15 \mathrm{~Hz}$. This comparison shows an error of $\epsilon_{f}=-0.25 \%$ for the results without damping and $\epsilon_{f}=0.65 \%$ for the cases with damping. Nevertheless, the FSI frequency is also very well predicted in both cases. One can notice that the frequency of the coupled system slightly increases due to the structural damping.

\begin{tabular}{|l||c|c|c|c|c|c|c|}
\hline \multicolumn{1}{|l||}{ Case } & \multicolumn{9}{|c|}{ Results } \\
\hline & $\mathrm{St}_{\mathrm{FSI}}(\mathrm{Hz})$ & $f_{\mathrm{FSI}}(\%)$ & Error & $\left.U_{y}^{*}\right|_{\max }$ & Error $(\%)$ & $\left.U_{y}^{*}\right|_{\min }$ & Error \\
\hline \hline Sim. (no damping) & 0.1125 & 7.08 & -0.25 & 0.456 & 9.1 & -0.464 & -10.6 \\
\hline Sim. (damping) & 0.1136 & 7.15 & 0.65 & 0.401 & -4.23 & -0.410 & 2.27 \\
\hline \hline Experiments & 0.1128 & 7.10 & - & 0.418 & - & -0.420 & - \\
\hline
\end{tabular}

Table 5: Comparison between numerical results with and without structural damping and the experiment.

\subsubsection{Phase-resolved flow field}

Owing to improved results in case of the structural damping, this case is chosen for the direct comparison with the measurements. The phase-averaging process delivers the phase-resolved flow fields. Four phase-averaged positions, which describe the most important phases of the FSI phenomenon, are chosen for the comparison: Figure 20 shows the flexible structure reaching a maximal upward deflection at $t \approx T / 4$. Then, it deforms in the opposite direction and moves downwards. At $t \approx T / 2$ the shell is almost in its undeformed state (see Fig. 21). Afterwards, the flexible structure reaches a maximal downward deformation at $t \approx 3 T / 4$ as seen in Fig. 22. At $t \approx T$ the period cycle is completed and the flexible structure is near its initial state presented in Fig. 23.

For each of the given phase-averaged positions, the experimental and numerical results (dimensionless streamwise and transverse velocity component) are plotted for comparison. Note that the structure in the experimental figures is shorter than in the simulation plots. Indeed, in the experiment it is not possible to measure exactly the whole experimental structure, around 1 
$\mathrm{mm}$ at the end of the structure is missing. As in Section 7.1 an additional figure shows the error between the simulation and the experiment for the velocity magnitude.

At $t \approx T / 4$ (see Fig. 20), when the structure is in its maximal upward deflection, the acceleration zone above the structure has reached its maximum. The acceleration area below the rubber plate is growing. Both phenomena are correctly predicted in the simulations. The computed acceleration area above the structure is slightly overestimated. However, the local error is mostly under $20 \%$.

The separation points at the cylinder are found to be in close agreement between measurements and predictions. Accordingly, also the location of the shear layers shows a good agreement between simulations and experiments. The shedding phenomenon behind the structure generates a turbulent wake, which is correctly reproduced by the computations. Owing to the phaseaveraging procedure, as expected all small-scale structures are averaged out.

At $t \approx T / 2$ (see Fig. 21), the shell is near its undeformed state. The acceleration zone above the structure has shrunk in favor of the area below the rubber plate. Regarding these areas the predictions show a very good agreement with the measurements (marginal local errors). The predicted wake directly behind the structure matches the measured one.

At $t \approx 3 T / 4$ (see Fig. 22), the downward deformation of the shell is maximal, the flow is the symmetrical to the flow observed at $t \approx T / 4$ with respect to $\mathrm{y} / \mathrm{D}=0$. Again the acceleration areas around the structure show a very good agreement with the measurements. Once more the wake is correctly predicted in the near-field of the structure.

At $t \approx T$ (see Fig. 23) the flow is symmetrical to the flow observed at $t \approx T / 2$ with respect to $\mathrm{y} / \mathrm{D}=0$. The computed acceleration area above the structure is slightly overestimated, but the local error is under $20 \%$. The wake is again correctly predicted except directly after the flexible structure.

For every position the local error is mostly under $20 \%$. In the error plot the areas with a bigger local error are near the structure and in the shear layers. This can be explained by the fact that near the structure and in the shear layers the gradients of the flow quantities are large. Since the mesh used for the simulation is much finer than the PIV measurement grid, the accuracy of the numerical solution is much higher than the precision of the PIV measurements in these regions. It is also difficult to measure the flow accurately in the direct vicinity of a body due to reflections of the laser light at the surface. Another reason is that the error expected by the PIV method is more important for low flow velocities. Close to the flexible structure and directly after its tail the flow velocity is small, which at least partially explains the deviations observed between the experimental and numerical results.

In summary, for every position the computed flow is in good agreement with the measured one. The shedding phenomenon behind the cylinder and the positions of the vortices convected downstream are correctly predicted.

\section{Available Data for Comparison}

The described benchmark FSI-PfS-1a is supposed to test, evaluate and improve numerical FSI codes. Therefore, the authors support all interested groups by the experimental and numerical data presented in this paper. For this purpose the data are made available on the ERCOFTAC Knowledge Base Wiki in the category 'Flow around Bodies' accessible as case 2-13 under

http://qnet-ercoftac.cfms.org.uk/w/index.php/UFR_2-13.

Available for comparison are:

- the data for the structural test cases described in Appendix A. 
- the 23 single phase-averaged two-dimensional reference velocity fields of the PIV measurement series and the corresponding numerical data used in Section 7.2;

- the raw and phase-averaged data of the displacement of the flexible structure.

- animations of the test case. 


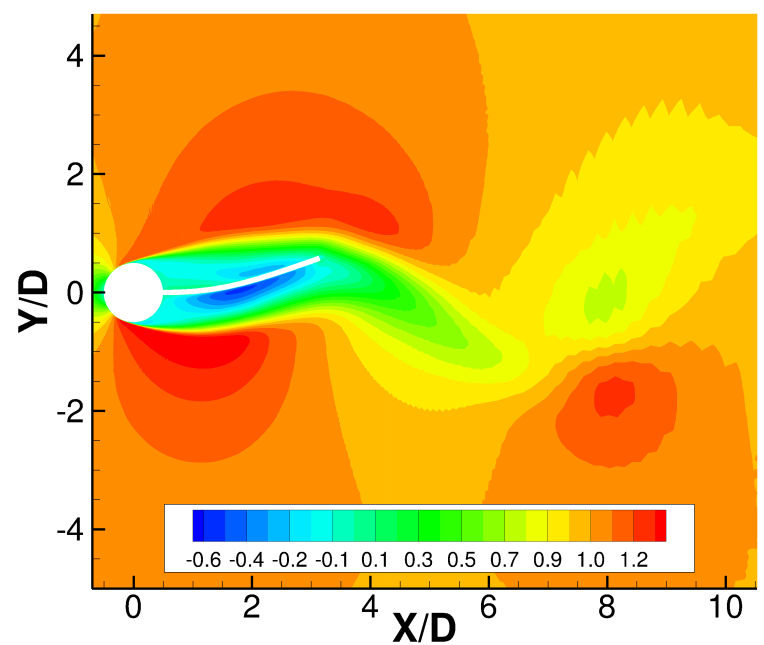

(a) Full case (streamwise velocity component)

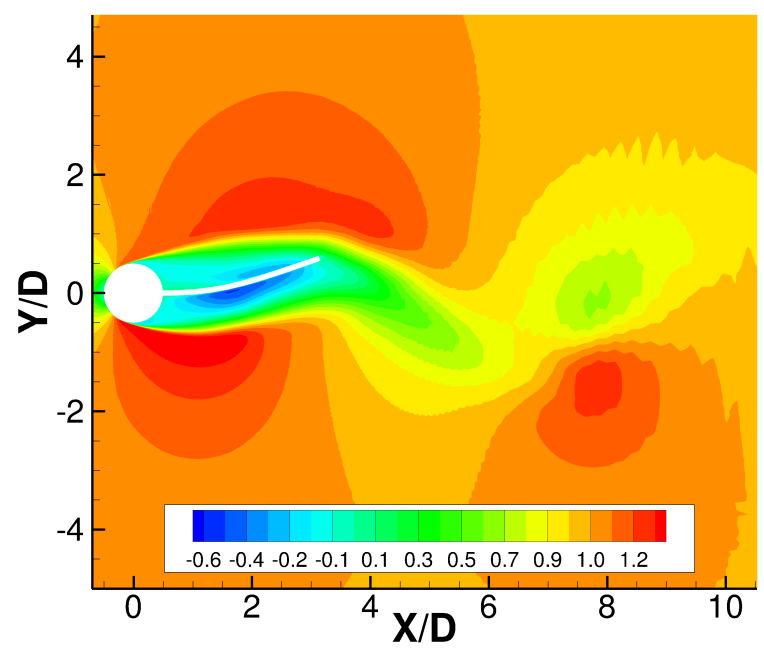

(c) Subset case (streamwise velocity component)

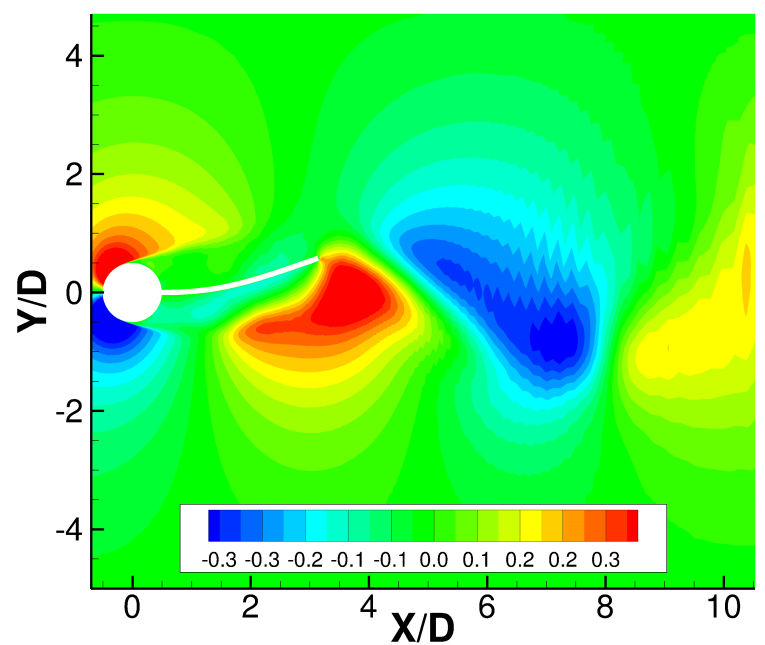

(b) Full case (transverse velocity component)

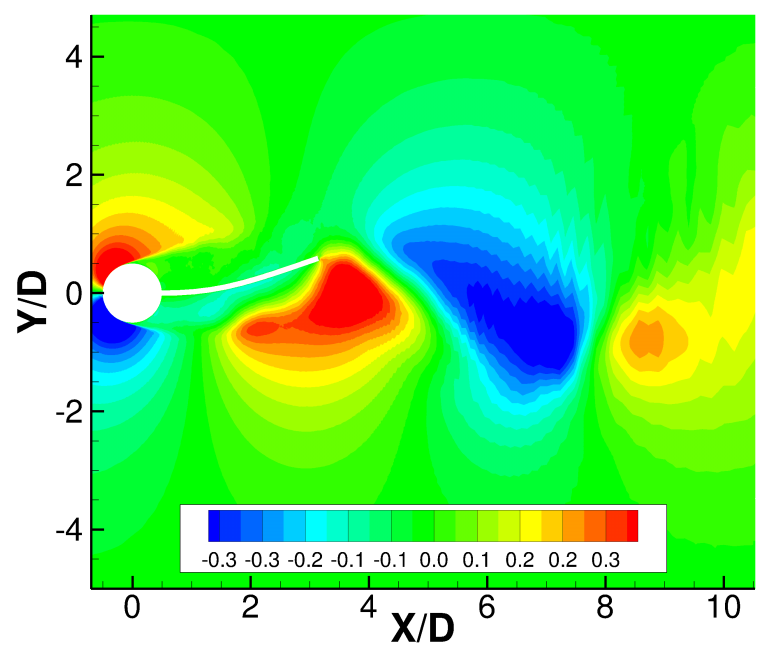

(d) Subset case (transverse velocity component)

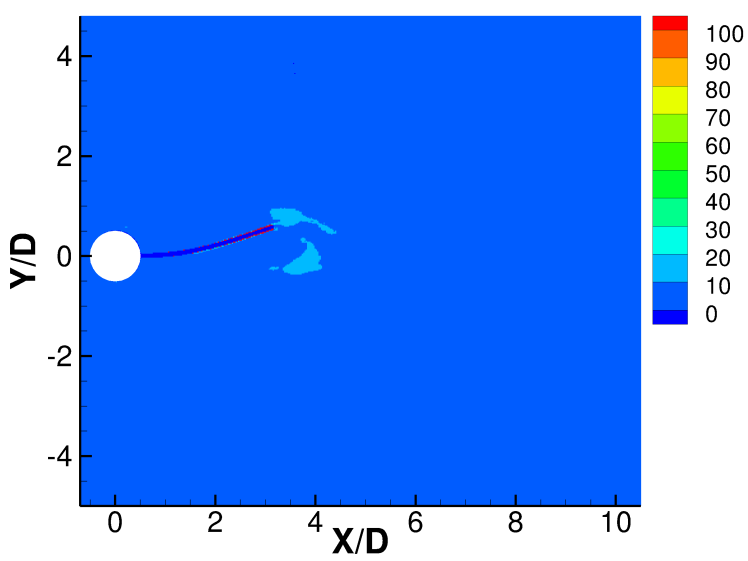

(e) Absolute local error (velocity magnitude) in \%

Figure 16: Comparison of the results for the full and subset case; phase-averaged data at $t \approx T / 4$. 


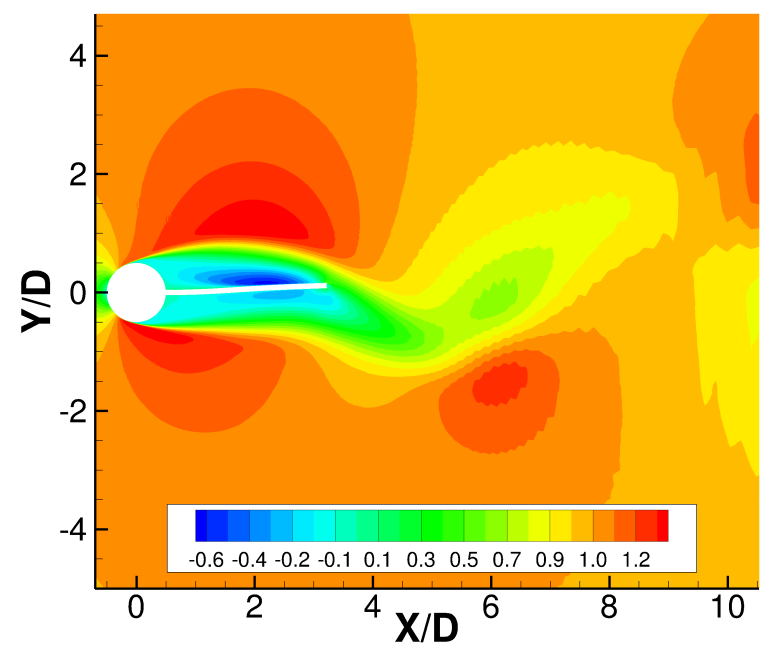

(a) Full case (streamwise velocity component)

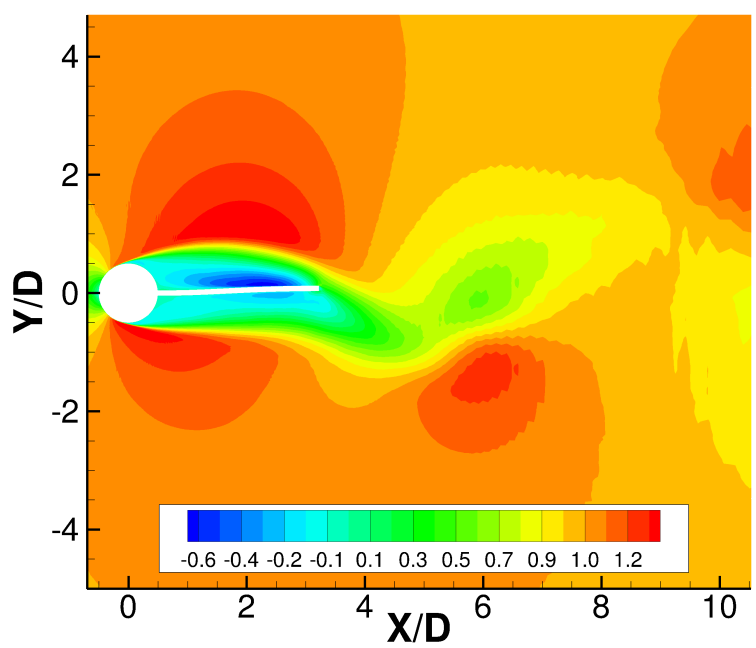

(c) Subset case (streamwise velocity component)

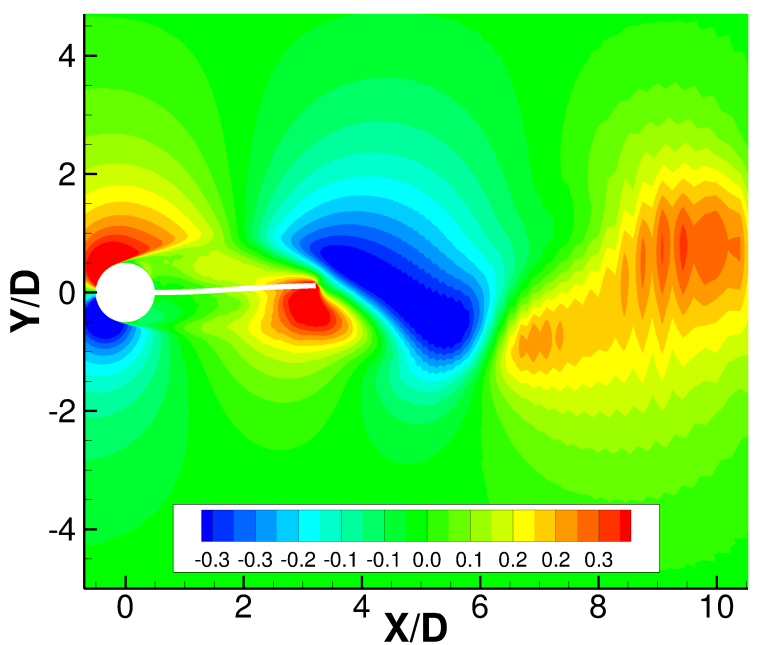

(b) Full case (transverse velocity component)

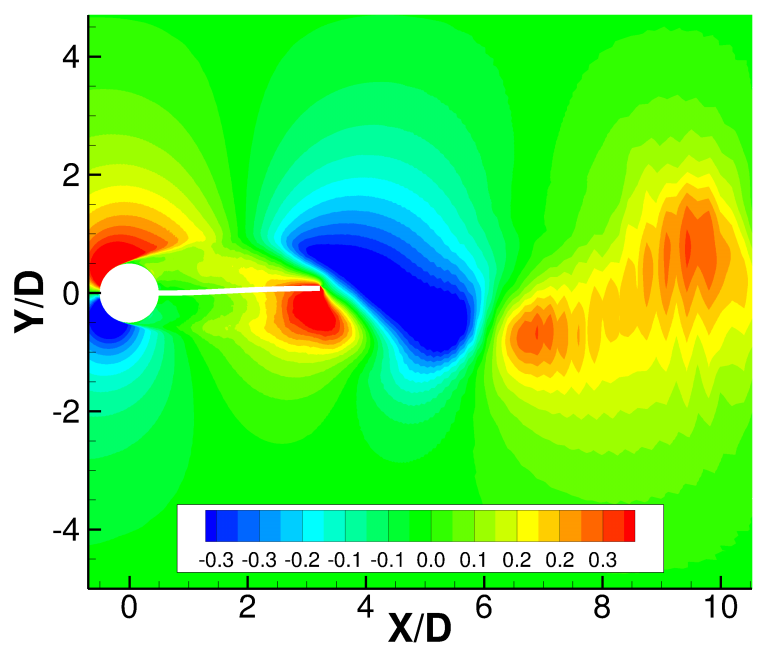

(d) Subset case (transverse velocity component)

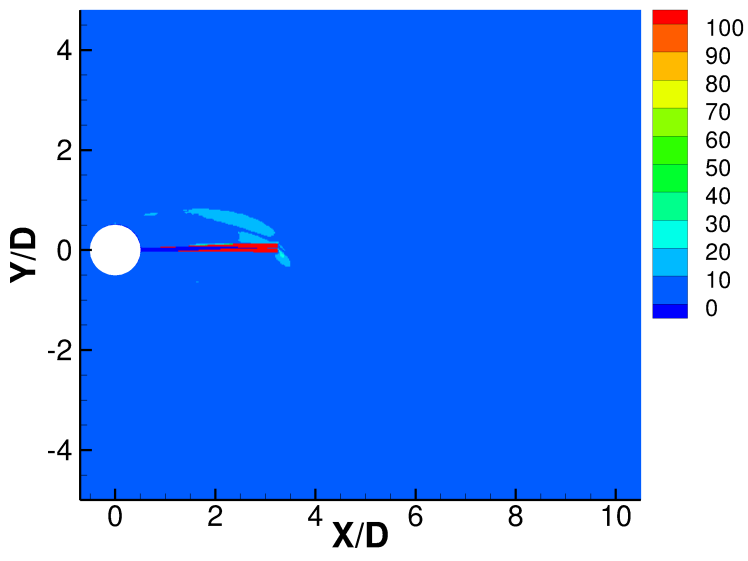

(e) Absolute local error (velocity magnitude) in \%

Figure 17: Comparison of the results for the full and subset case; phase-averaged data at $t \approx T$. 


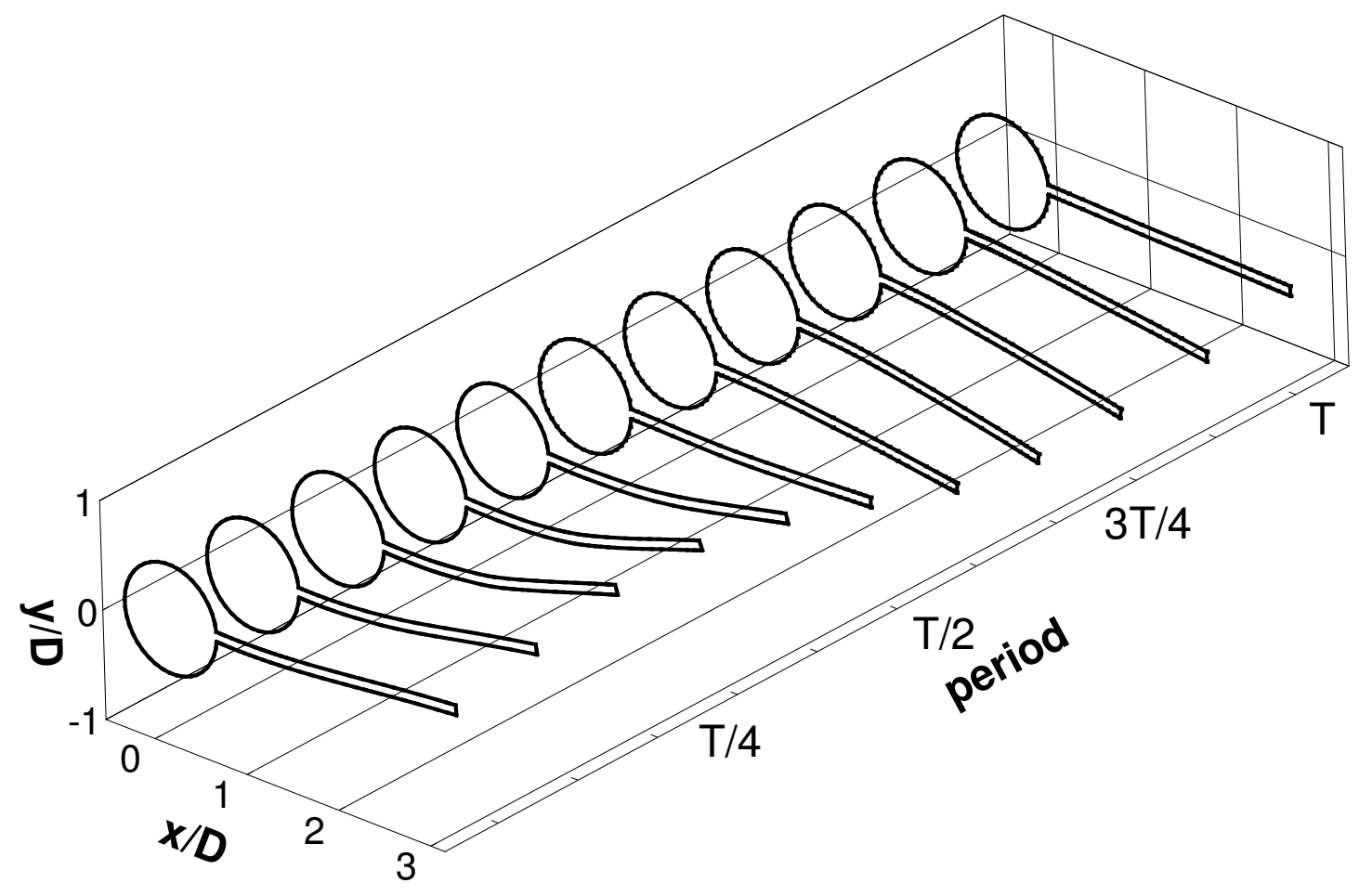

Figure 18: Experimental structural results: Structure contour for the reference period. 


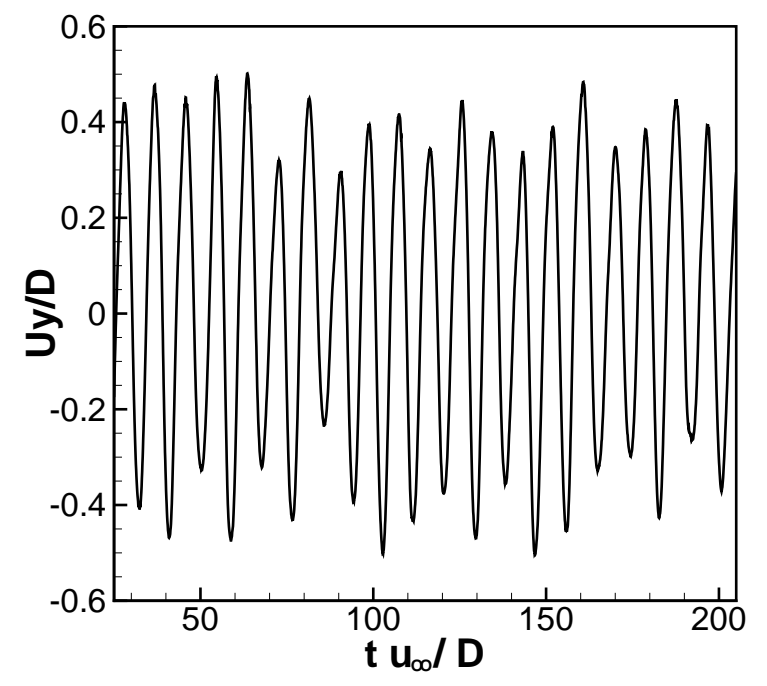

(a) Experimental raw signal: Dimensionless displacement.

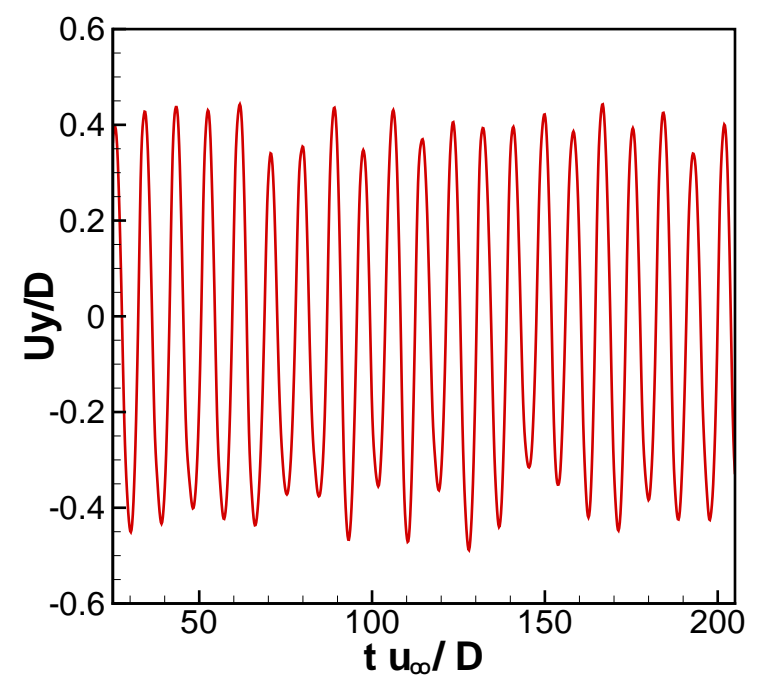

(c) Numerical raw signal (with damping): Dimensionless displacement.

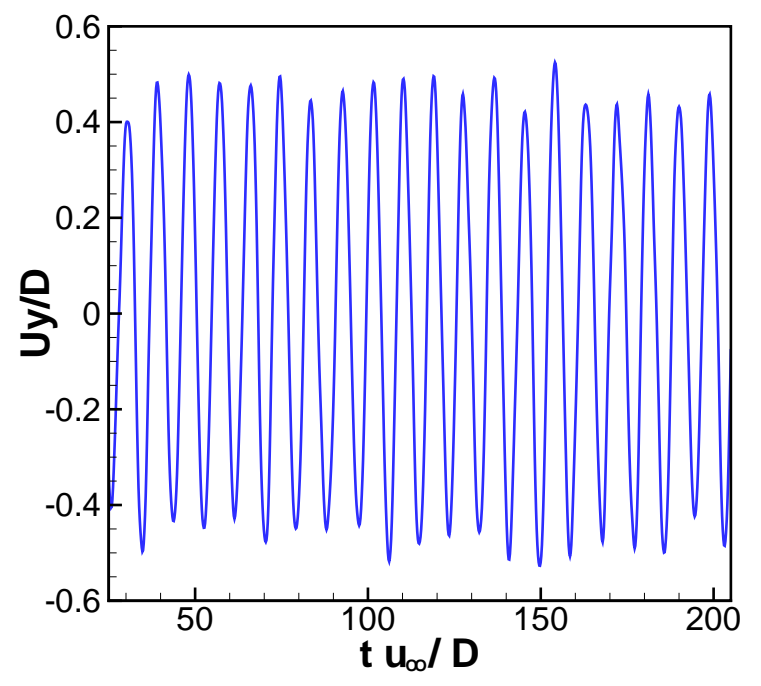

(b) Numerical raw signal (without damping): Dimensionless displacement.

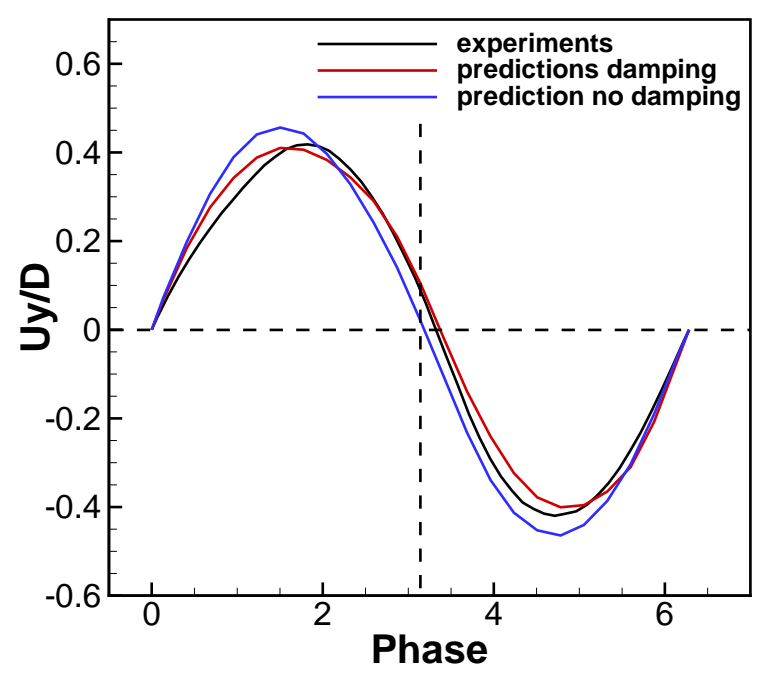

(d) Comparison of the averaged phase of the experimental and numerical data.

Figure 19: Comparison of experimental and numerical results; raw signals and averaged phases of a point located in the midplane at $9 \mathrm{~mm}$ distance from the shell extremity. 


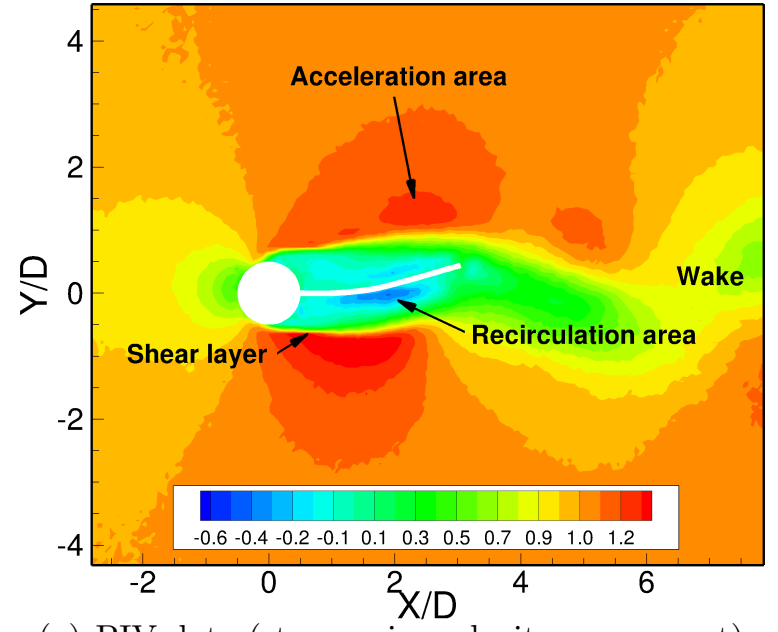

(a) PIV data (streamwise velocity component)

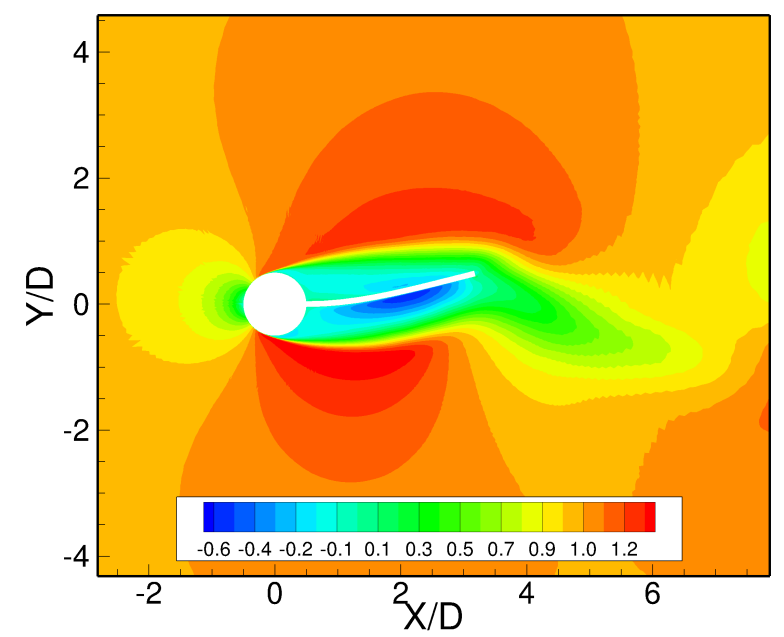

(c) FSI simulation (streamwise velocity component)

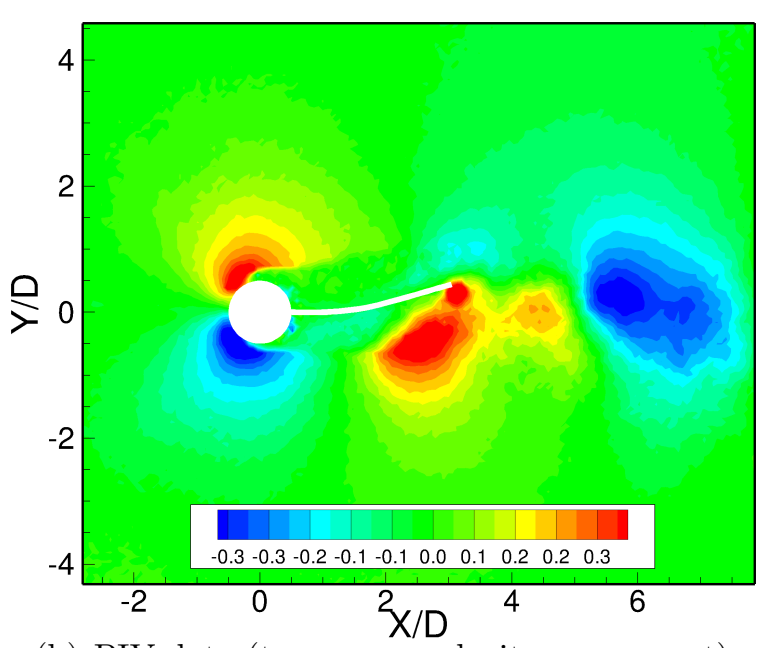

(b) PIV data (transverse velocity component)

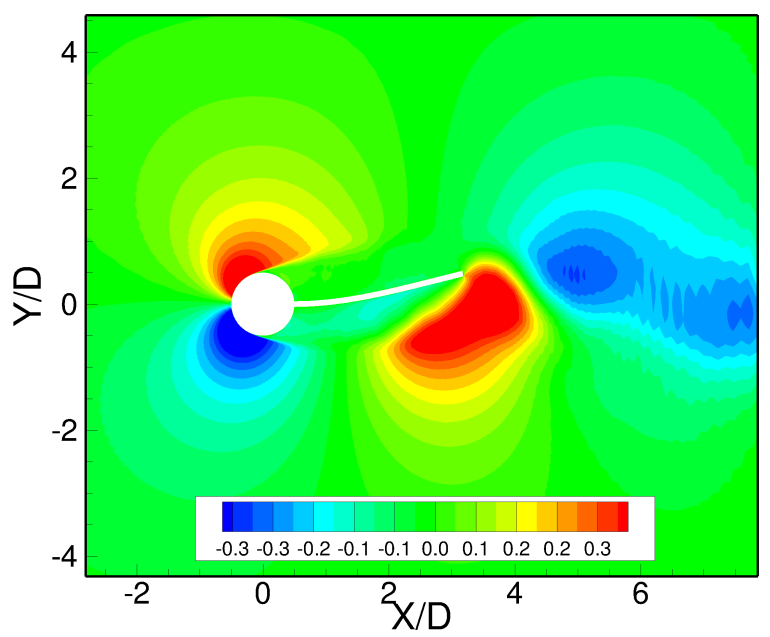

(d) FSI simulation (transverse velocity component)

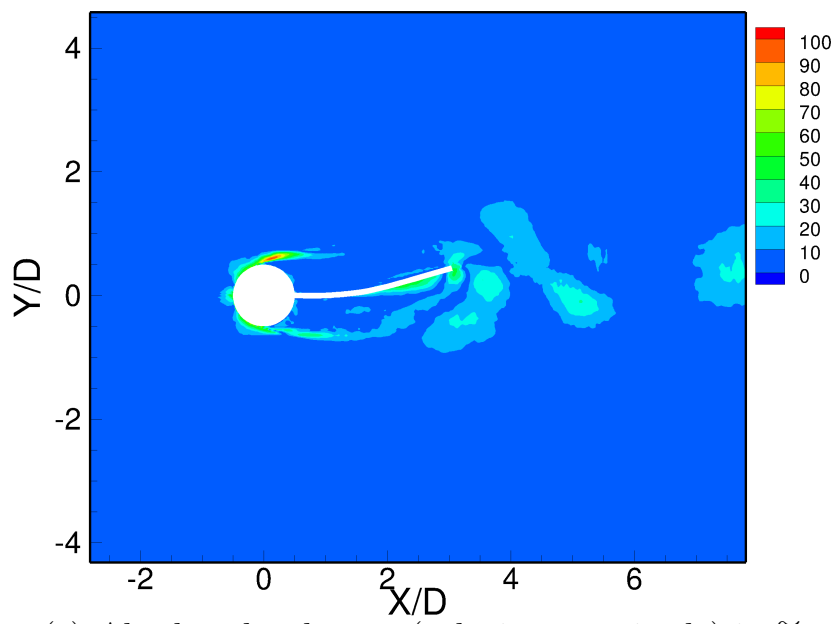

(e) Absolute local error (velocity magnitude) in \%

Figure 20: Comparison of experimental and numerical results (subset case with damping, see Table 5); phase-averaged data at $t \approx T / 4$. 


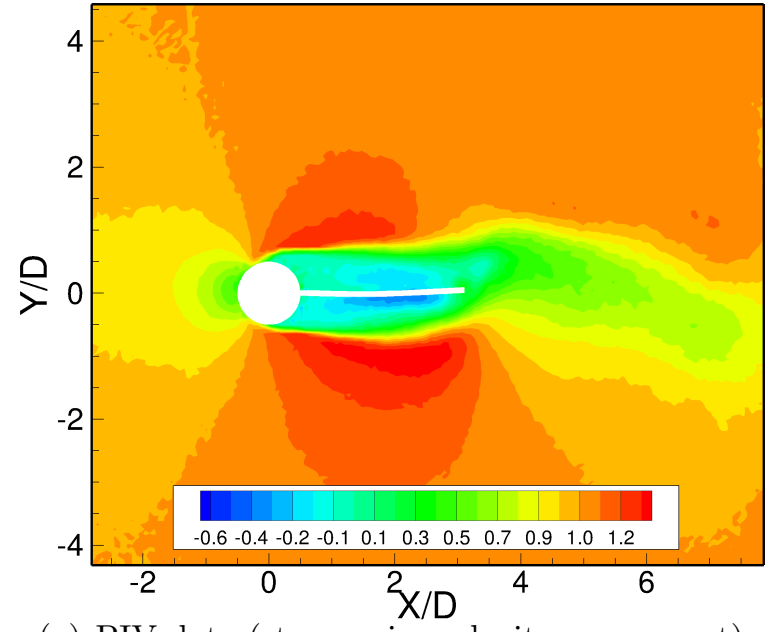

(a) PIV data (streamwise velocity component)

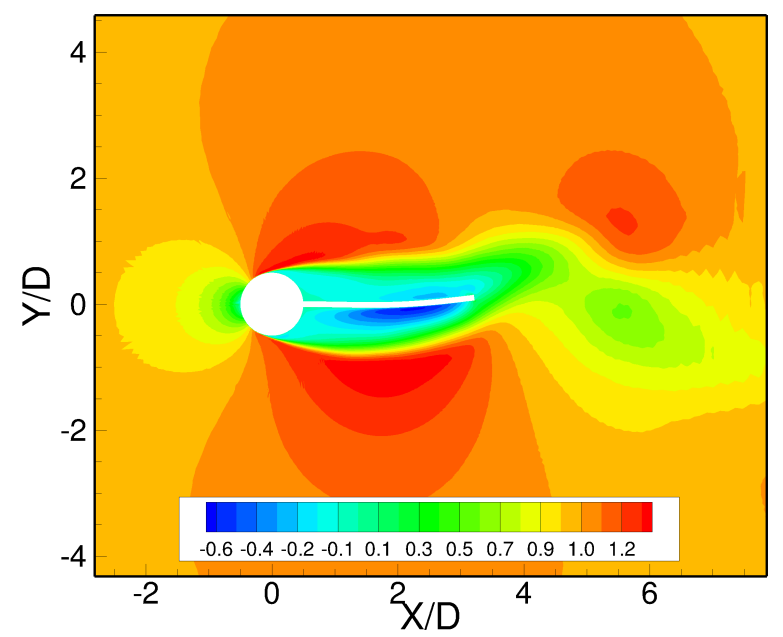

(c) FSI simulation (streamwise velocity component)

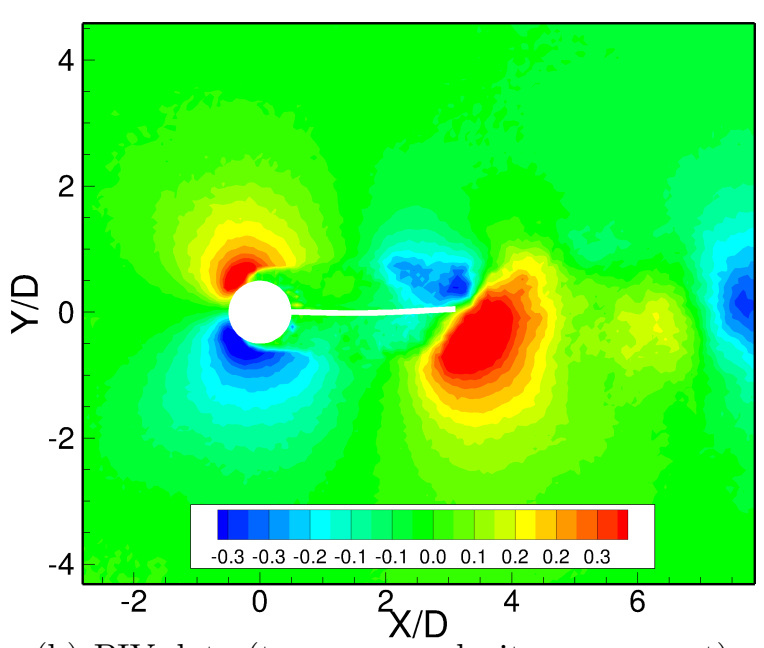

(b) PIV data (transverse velocity component)

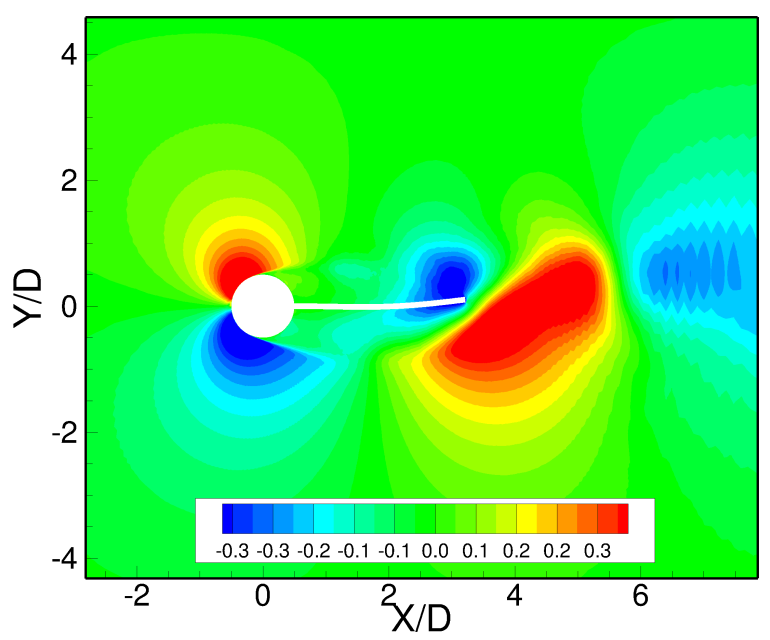

(d) FSI simulation (transverse velocity component)

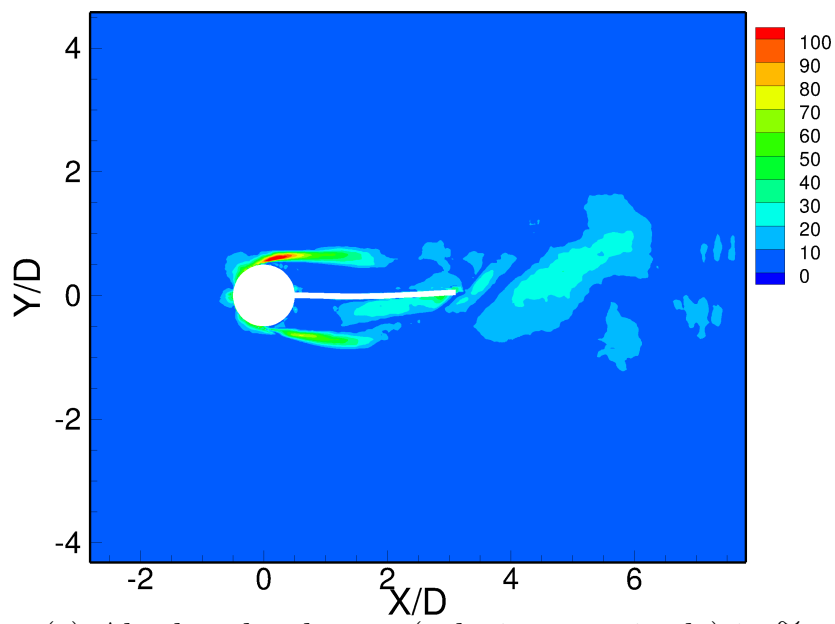

(e) Absolute local error (velocity magnitude) in \%

Figure 21: Comparison of experimental and numerical results (subset case with damping, see Table 5); phase-averaged data at $t \approx T / 2$. 


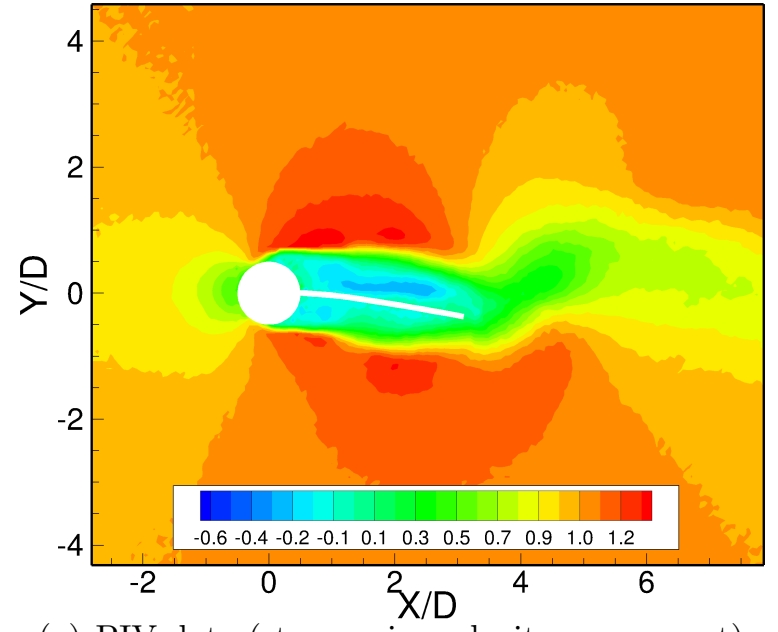

(a) PIV data (streamwise velocity component)

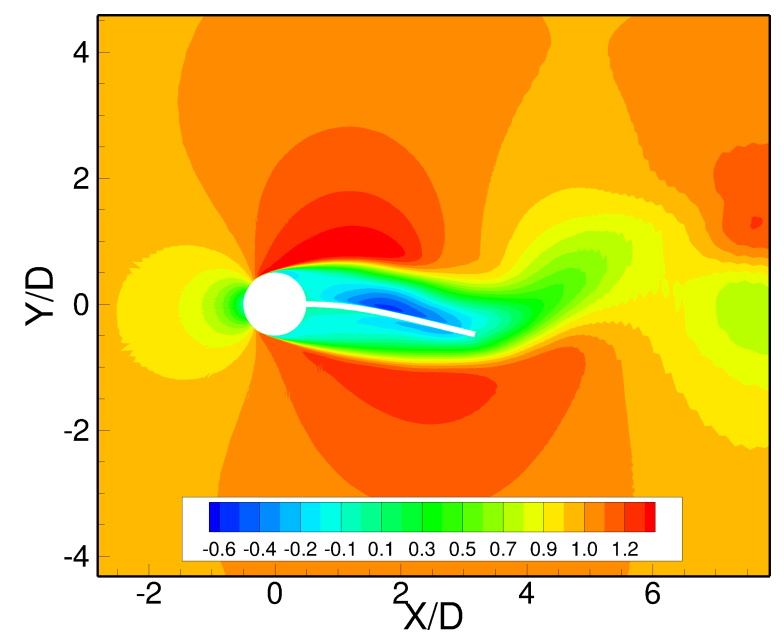

(c) FSI simulation (streamwise velocity component)

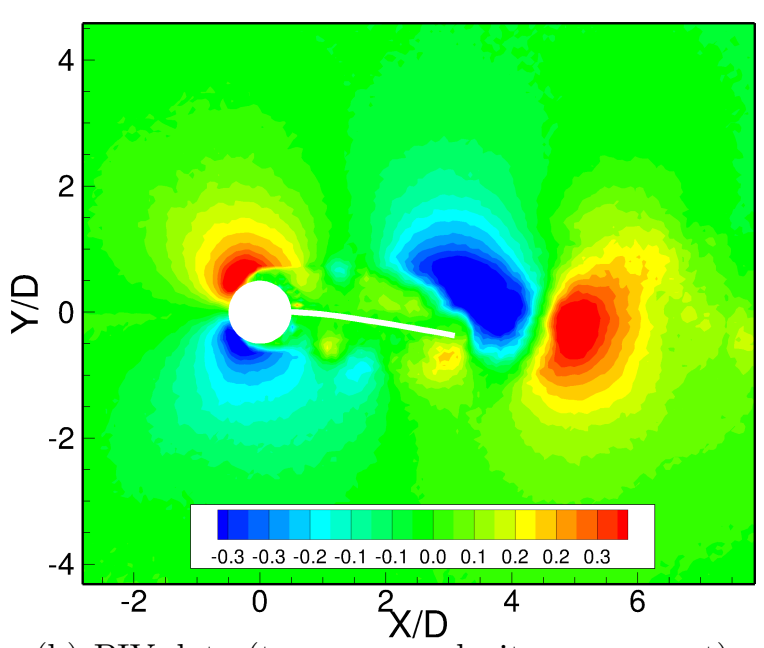

(b) PIV data (transverse velocity component)

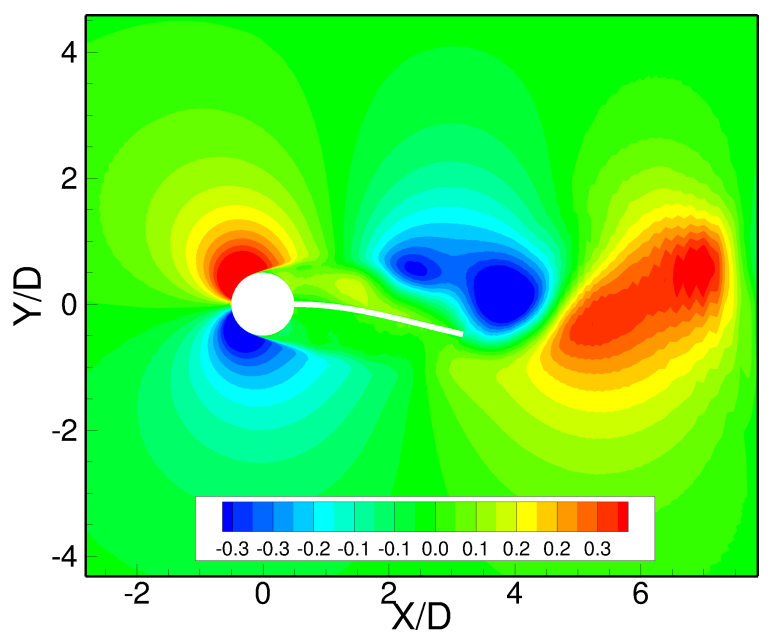

(d) FSI simulation (transverse velocity component)

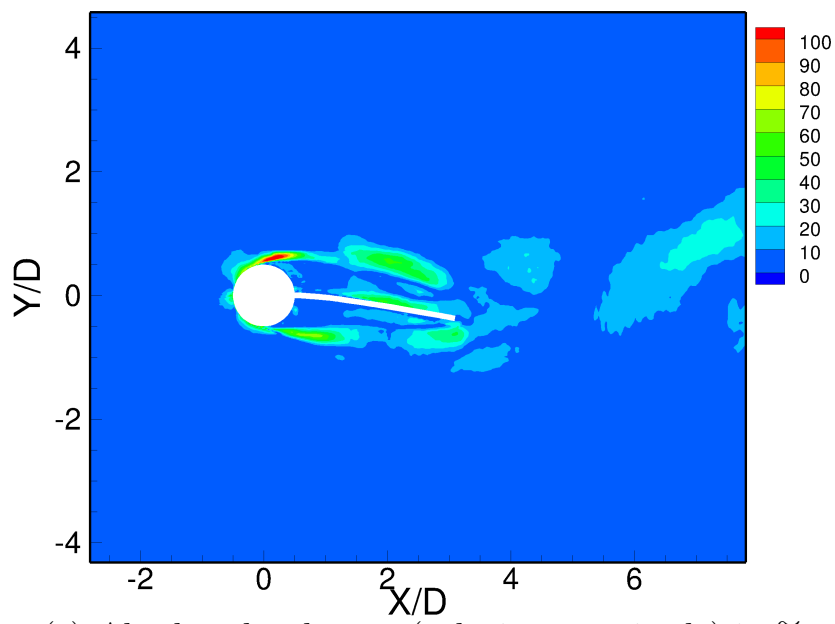

(e) Absolute local error (velocity magnitude) in \%

Figure 22: Comparison of experimental and numerical results (subset case with damping, see Table 5); phase-averaged data at $t \approx 3 T / 4$. 


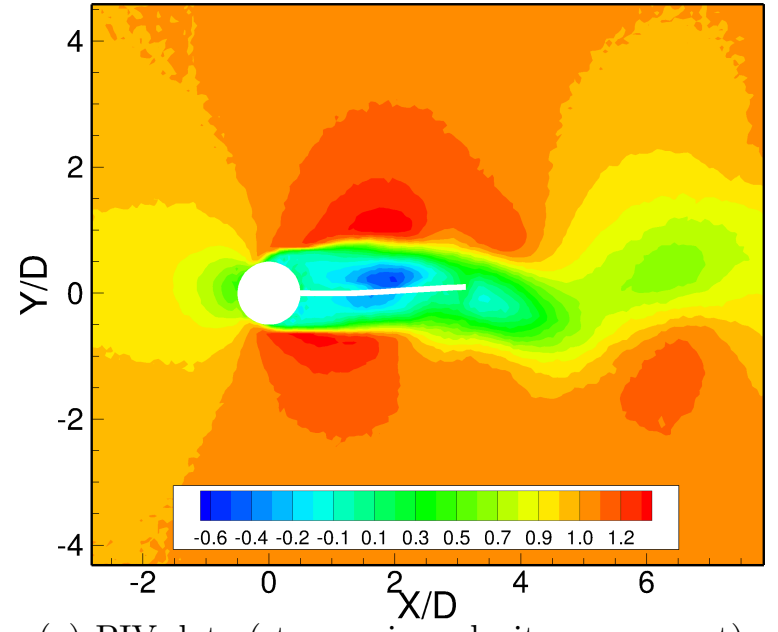

(a) PIV data (streamwise velocity component)

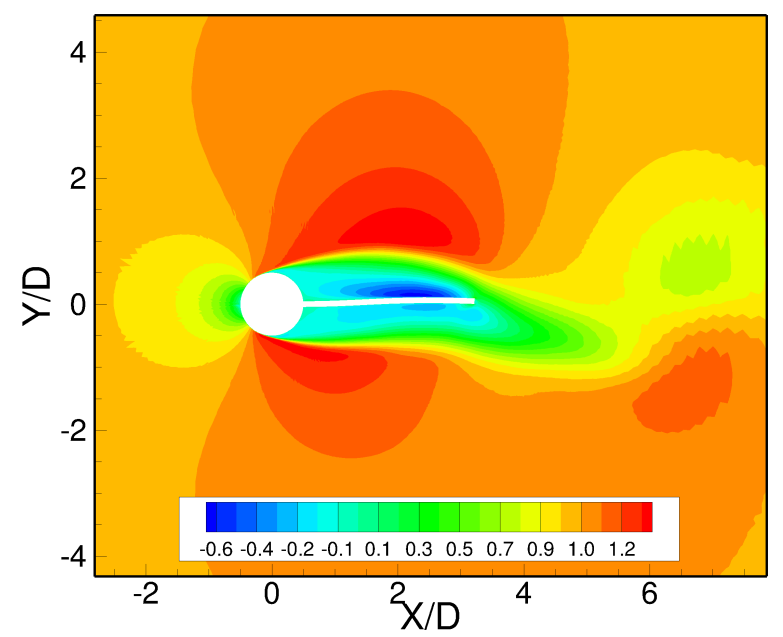

(c) FSI simulation (streamwise velocity component)

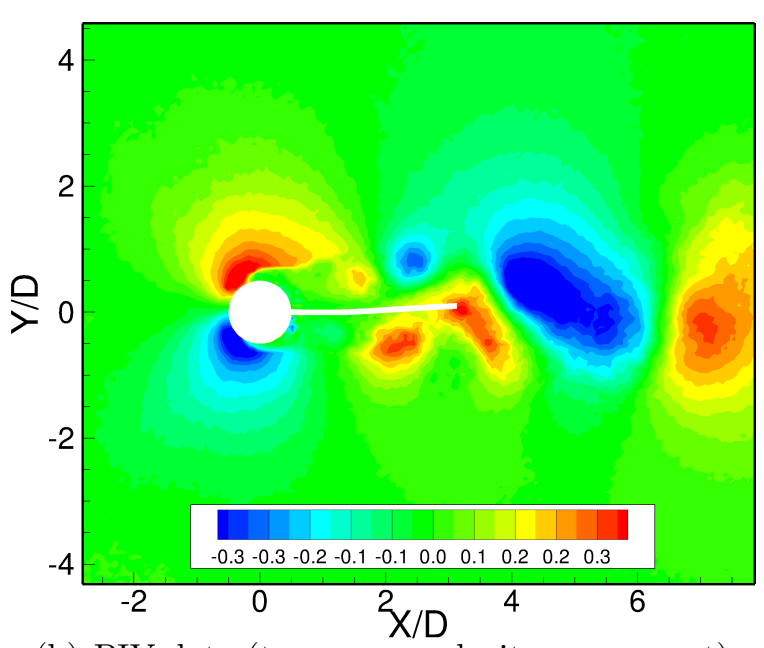

(b) PIV data (transverse velocity component)

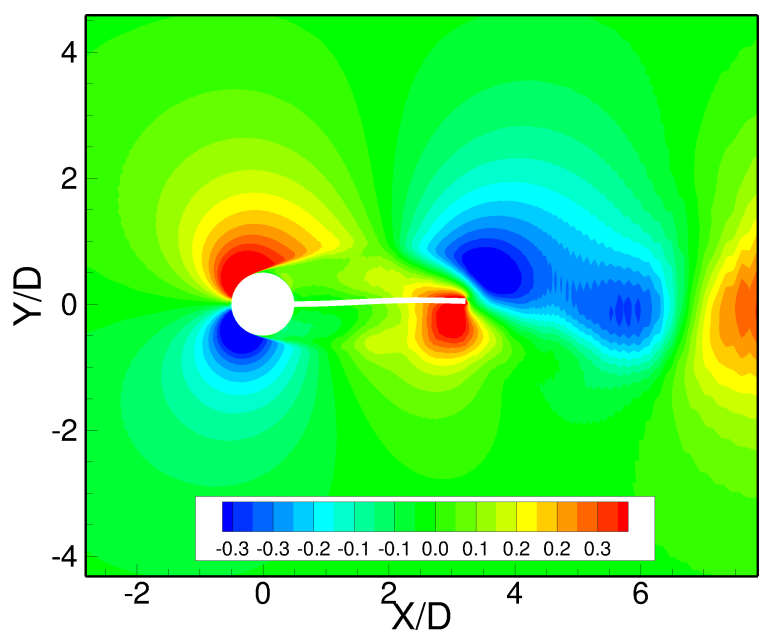

(d) FSI simulation (transverse velocity component)

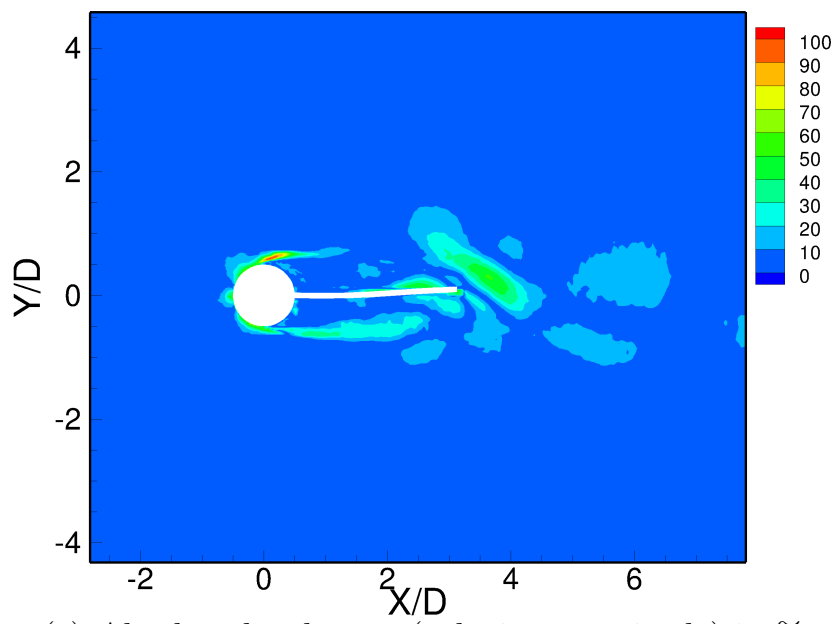

(e) Absolute local error (velocity magnitude) in \%

Figure 23: Comparison of experimental and numerical results (subset case with damping, see Table 5); phase-averaged data at $t \approx T$. 


\section{Conclusions}

A new FSI validation test case denoted FSI-PfS-1a is proposed. The definition of the test case is driven by the idea to setup a well-defined but nevertheless challenging validation test case for fluid-structure interaction in the turbulent flow regime. A rigid front cylinder and a flexible membranous rubber tail attached to the backside of the cylinder form the structure which is exposed to a uniform inflow at a low turbulence level. Thus three critical issues of precursor benchmarks are circumvented, i.e., an additional degree of freedom of a rotating front cylinder, an extremely thin flexible structure and an additional weight at the end of the membranous structure. The investigations comprise three parts.

First, two dynamic structural tests were carried out experimentally and numerically in order to evaluate an appropriate material model and to check and evaluate the material parameters of the rubber (Young's modulus, structural damping). This preliminary work has shown that the St. Venant-Kirchhoff material model is sufficient to describe the deflection of the flexible structure.

Second, detailed experimental investigations in a water tunnel using optical measurement techniques for both, the fluid flow and the structure deformation, were carried out. A quasi-periodic oscillating flexible structure in the first swiveling mode with a corresponding Strouhal number of about $\mathrm{St}_{\mathrm{FSI}}=0.11$ is found. A post-processing of the extensive data sets delivered the phase-averaged flow field and the structural deformations.

Third, various simulations relying on a newly developed FSI simulation tool combining a partitioned solution strategy with an eddy-resolving scheme (LES) were performed. A subset case and full case are taken into account. Owing to the wider structure and less constraints of the lateral nodes the deformations in the spanwise direction were found to be larger in the full case reflecting some kind of mild waves in the structure. Nevertheless, in relation to the deformation of the structure in cross-flow direction the spanwise deflections are insignificant, especially for the comparison of the phase-averaged signals.

A study on three parameters for the subset case without structural damping yields that the Young's modulus (or the Cauchy number) has a very important influence on the system. It controls in which swiveling mode the flexible structure oscillates. The thickness of the rubber plate $\mathrm{h} / \mathrm{D}$ plays a role in the results, too, but not so significant as the Young's modulus. The parameter with the least effect on the FSI simulations is the density ratio of the body: limited variations of the density do not have major influence on the predictions.

As usual for rubber material, a certain level of structural damping has to be expected. To model this phenomenon in a simple and straightforward way, classical Rayleigh damping is used and adjusted based on one of the pure structural tests presented in the appendix. The FSI simulations with and without structural damping are compared with the experiment. It turns out that the structural damping can not be ignored in the present case and significantly affects the deflection of the structure. Without taking the damping into account the structural deflections are overpredicted. Including the simple damping model improves the results. The eddy-resolving FSI simulations are found to be in close agreement with the experiment for every position of the flexible structure. Merely the amplitudes of the deflections are slightly underpredicted with damping. Nevertheless, the shedding phenomenon behind the cylinder/structure and the positions of the vortices convected downstream are correctly predicted. Furthermore, the FSI frequency found in the simulations matches particularly well the measured one.

The described test case FSI-PfS-1a is a part of a series of reference test cases designed to improve numerical FSI codes. A second test case FSI-PfS-2a is described in Kalmbach and Breuer (2013). The geometry is similar to the first one: A fixed rigid cylinder with a plate clamped behind it. However, this time a rear mass is added at the extremity of the flexible 
structure and the material (para-rubber) is less stiff. The flexible structure deforms in the second swiveling mode and the structure deflections are completely two-dimensional and larger. For each test case all the experimental data are available on demand.

\section{Acknowledgments}

The project is financially supported by the Deutsche Forschungsgemeinschaft under the contract numbers BR 1847/12-1 and BL 306/26-1. The computations were partially carried out on the German Federal Top-Level Computer SuperMUC/SuperMIG at LRZ Munich under the contract number pr47me. We would like to thank the Draftex Automotive GmbH in Grefrath for providing the rubber material and the corresponding measurements of its characteristic properties. Special thanks goes also to H. Lienhart (LSTM Erlangen) for the construction of the water channel and many helpful advices regarding the experimental setup. All kinds of support are gratefully acknowledged.

\section{Appendix A. Description of pure structural tests}

The measurements of the different structural test cases and the respective numerical simulations with different parameter sets serve two different purposes. On the one hand, the characteristic model parameters should be determined and checked by parametric studies. On the other hand, it should be verified that with the chosen material models, the correct structural behavior can be recovered in the predictions.

Both pure structural cases consist of a flexible rubber plate $(0.06 \mathrm{~m} \times 0.177 \mathrm{~m} \times 0.0021 \mathrm{~m})$ clamped between a rigid cylinder $\left(\rho_{\text {cylinder }}=3146 \mathrm{~kg} / \mathrm{m}^{3}\right)$ and two half cylinders made of brass $\left(\rho_{\mathrm{bar}}=8450 \mathrm{~kg} / \mathrm{m}^{3}\right)$ at the other end. These constrain the respective two edges of the plate to deform without warping, which results in a clear and easy to measure deformation state. The test cases use a setup with a vertical alignment of the plate (see Fig. A.24).

Free oscillation test.

In the first test case, the cylinder (C1) is fixed. To initiate an oscillation, the free end is deflected by a well-defined horizontal movement $U_{y}$ and then released, such that a decaying vibration can be accurately traced using the laser distance sensor described in Section 3.2.

Forced periodic oscillation test.

For the second test scenario, the support of the cylinder $(\mathrm{C} 1)$ at the upper margin is a rotatable bearing. This cylinder is stimulated by a periodic excitation of $\phi(t)=\phi_{\max } \sin (\omega t)+\phi_{\text {offset }}$ with a maximal angular deflection of $\phi_{\max }=6.1^{\circ}$ and a frequency of $\omega=18.33 \mathrm{~s}^{-1}$ resulting in an unsteady deformation of the rubber plate. The offset of $\phi_{\text {offset }}=+0.5^{\circ}$ is required because of the experimental setup of the crank drive that creates a slightly asymmetric angular excitation of the actuated upper cylinder. However, since this shift can be easily deducted from the measurements, it is not mentioned in the following. The inertia of the end bar increases the displacements and also modifies the deflection behavior of the flexible structure to nonlinear deformations. The low oscillation frequency is chosen to reduce the velocity-related deformation effects of the rubber material. As before the laser distance sensor described in Section 3.2 detects the displacements with a high measuring frequency. 


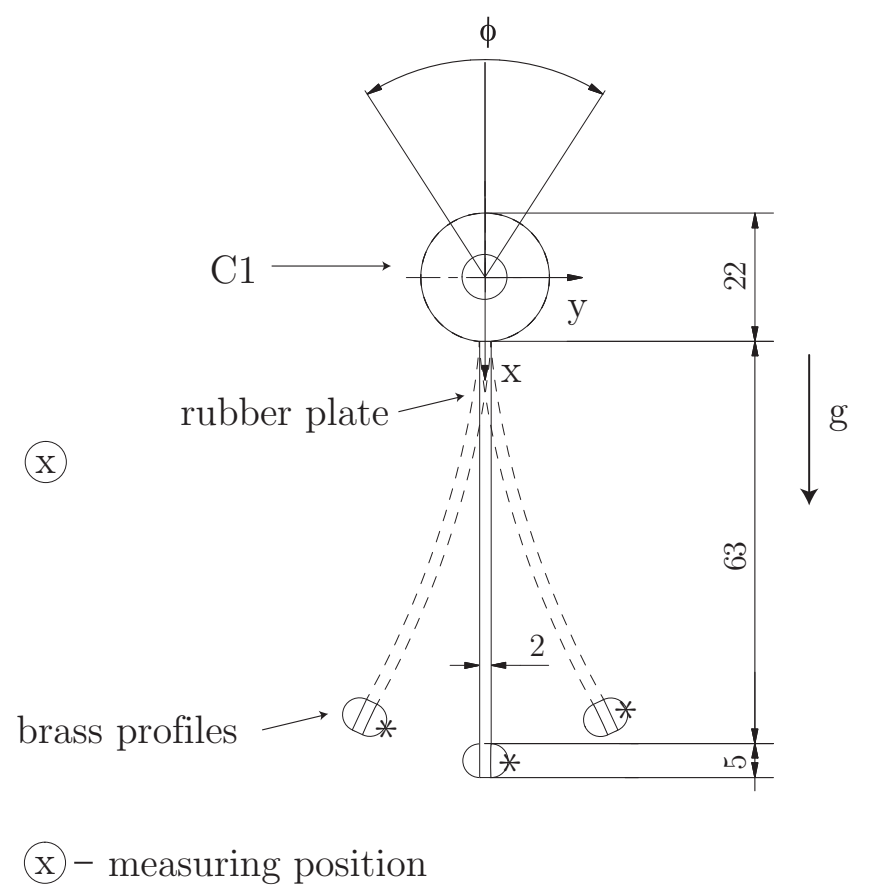

Figure A.24: Sketch of the geometrical configuration of the structural tests.

\section{Appendix B. Validation of the structural model}

To demonstrate the applicability of solid as well as shell elements for the structural finiteelement model, comparative simulations were carried out for the structural test cases mentioned in Appendix A. In order to separate the effects of structural damping, the cross-comparison of models was done via the computation of eigenfrequencies and the corresponding structural eigenmodes which are shown in Fig. B.25. Note that the shell models are visualized with their thickness to demonstrate the regions of the more bulky parts. It can be seen that the main dynamic deformation mechanisms which are relevant for the FSI benchmark (e.g., the first and second plate bending modes instead of thickness vibration modes) are perfectly recovered by the solid as well as by the dimensionally reduced shell model. Special care was taken to model the translational and rotational inertia of the cylindrical weight appropriately for the shell setup. As can be immediately concluded from the fact of a pure two-dimensional discretization compared to a full resolution of the three-dimensional body, the computations using shell elements were 8 times faster and thus, remarkably less computationally expensive. For the correct reproduction of the transient structural test cases, the consideration of the pre-stressing effect of the cylinder's weight is crucial and may not be neglected. This issue was investigated by doing eigenfrequency evaluations with and without considering the weight of the cylinder: In the former case, first a static simulation of the benchmark setup with self-weight was carried out and the resulting stresses were transferred to the initial structural configuration for the eigenfrequency analysis. As a consequence, the measured first eigenfrequency of about $4.55 \mathrm{~Hz}$ was computed as $4.69 \mathrm{~Hz}$, whereas without consideration of the pre-stress induced by self-weight the stiffness of the structure was under-estimated and thus the first eigenfrequency was determined to be about $4.16 \mathrm{~Hz}$.

Due to the usual dynamic behavior of rubber, a certain level of damping has to be expected. To represent this adequately in the numerical simulation, various approaches are possible. However, damping in a vibrating structure is a complex phenomenon with various sources (Petersen, 2000). The goal within this benchmark description is to provide a simple modeling 

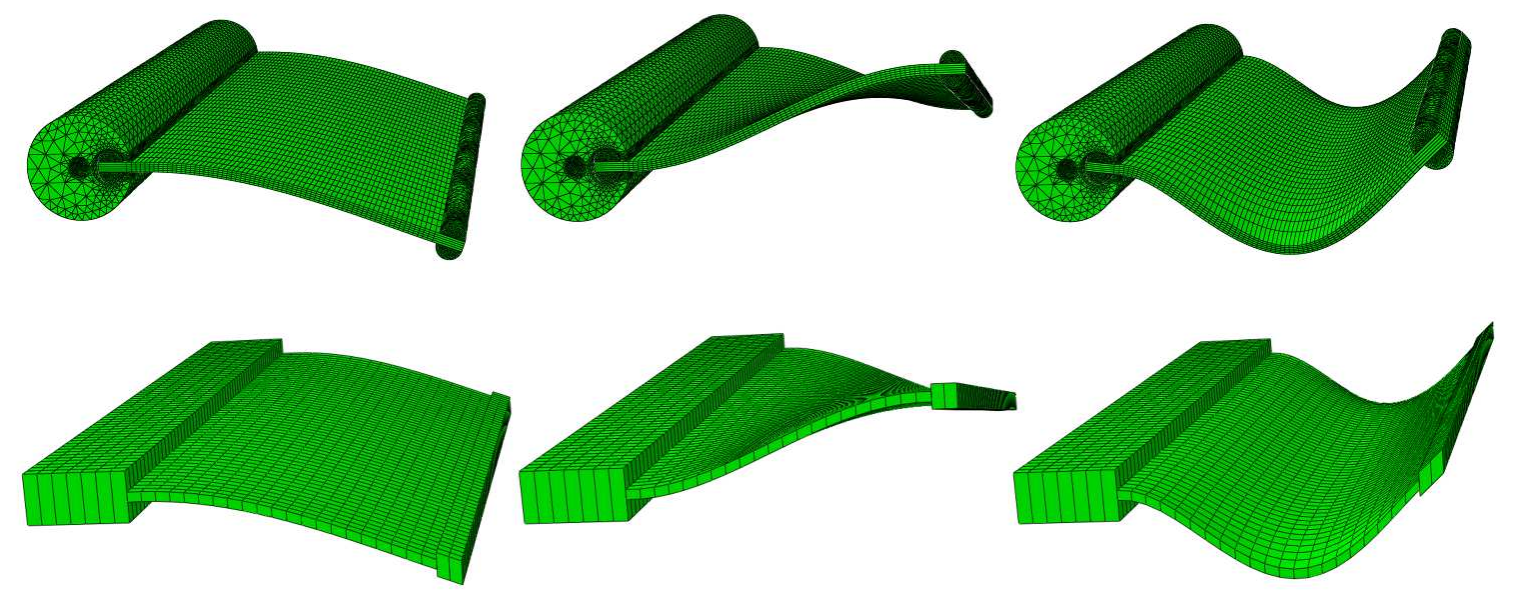

$1^{\text {st }}$ bending mode

$1^{\text {st }}$ twist mode

$2^{\text {nd }}$ bending mode

Figure B.25: Comparison of the first three structural eigenmodes for solid (upper row) as well as shell models (lower row) for the pure CSD setup presented in Appendix A.

for the damping effects to enable the computation with standard finite-element codes without adapting them. Moreover, based on the experimental observations it can be observed, that within the proposed FSI benchmark only the lowest eigenmodes (nearly exclusively the first bending mode shown in Fig. B.25) of the structure are excited. A proper choice for the damping model is therefore the Rayleigh damping: all damping effects are summarized in a velocity proportional damping matrix $\mathbf{D}$ which is composed of a linear combination of the mass matrix $\mathbf{M}$ and the (initial) stiffness matrix $\mathbf{K}_{\mathbf{0}}$ :

$$
\mathbf{D}=\alpha \mathbf{M}+\beta \mathbf{K}_{0} .
$$

According to Clough and Penzien (1993), the two open Rayleigh damping factors $\alpha$ and $\beta$ can be evaluated by solving a pair of equations, in case that two damping ratios and the respective frequencies are known. Since the FSI test case shows nearly only oscillations in the first eigenmode, it was decided to take only one damping factor. More precisely, the stiffness proportional damping factor $\beta$ is considered to be evaluated and the mass proportional quantity $\alpha$ is assumed to be zero. The reason for this decision is that the stiffness proportional damping is considered to be more realistic (see, e.g., Krätzig et al., 1996) in this case, since the mass proportional damping factor results in decaying damping effects on higher modes. The damping parameter $\beta$ is calibrated with the free oscillation test, introduced in Appendix A and it is assumed that it can be used for the FSI simulations accordingly.

\section{Free oscillation test.}

In order to compute this test Carat++ uses a grid with $10 \times 10$ quadrilateral 7-parameter shell elements mentioned above. The generalized- $\alpha$ method is used with a time step of $4 \times 10^{-3} \mathrm{~s}$. Abaqus uses the meshes presented in Fig. B.25. The Abaqus shell elements are S8R. The HHT time discretization is set with an initial time step of $1 \times 10^{-3} \mathrm{~s}$. Then, the adaptive time step control is enabled with TRANSIENT FIDELITY.

Based on some rough estimations the value could be found to be in the range between 0.01 and 0.02. Further parametric studies and cross-comparisons with the experimental data lead to a value $\beta=0.017$. Computed results for the free oscillation test and the measured deformations are displayed in Fig. B.26. It can be seen that with this choice of the damping parameters 
the experimentally observed decay behavior of the oscillation due to the initial deflection is captured very well.

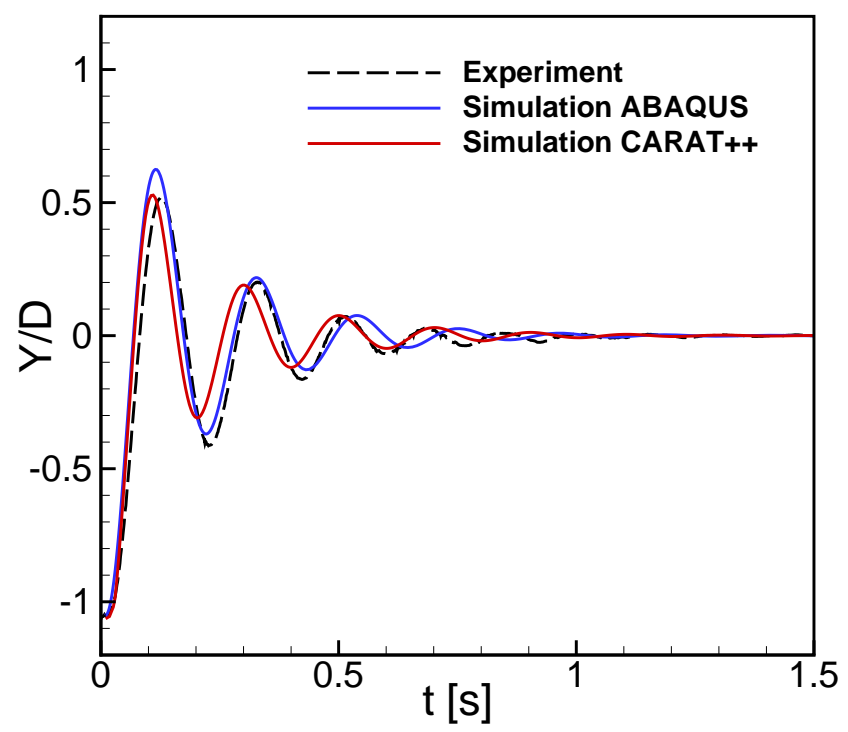

Figure B.26: Free oscillation test: Comparison of y-deformations of the point marked as measuring position in Fig. A.24 between structural simulations and experiment.

Forced periodic oscillation test.

The forced periodic oscillation test is also computed with Abaqus using shell and solid finite elements with the settings mentioned above and the material parameters provided. The simulation results of the shell model are exemplary compared to the measured deformations in Fig. B.27. A time period of a fully developed oscillation is chosen, to avoid transient effects during the startup procedure of the experiment. Structural damping is used in the simulation and adjusted as described above. The very good agreement of numerical and experimental data supports the chosen material data summarized in Table 3. Thus the structural modeling as an important part of the overall FSI setup is carefully checked.

\section{Appendix C. Grid dependency study for the structural model}

As a preliminary investigation for the choice of a proper structure mesh, a small test case with a constant pressure distribution on one plate surface is carried out using the material parameters defined in Section 2.4. The magnitude of the pressure is chosen such that the final tip deformation is in the range of the maximally expected tip deflection of the flexible structure in the FSI case. This load scenario is computed with a sequence of systematically refined meshes (using quadrilateral four-node shell elements) for the quadratic structure used in the subset case and for the rectangular one used in the full case. The tip deformations obtained are summarized in Table C.6. Moreover, a cross-check by a simulation using a very fine mesh of solid elements shows a deviation in the tip displacement of less than 1\%. As a result of this grid independency study, a mesh with 30 shell elements in streamwise direction can be considered as converged for both cases. Astonishingly, even a mesh with only 10 shell elements of the used type in streamwise direction could be seen as reasonable for a deflection comparable to the one observed in the FSI experiments. Thus in order to save CPU-time 10 $\times 10$ and $10 \times 30$ shell elements are applied in the FSI prediction of the subset and the full case, respectively. 


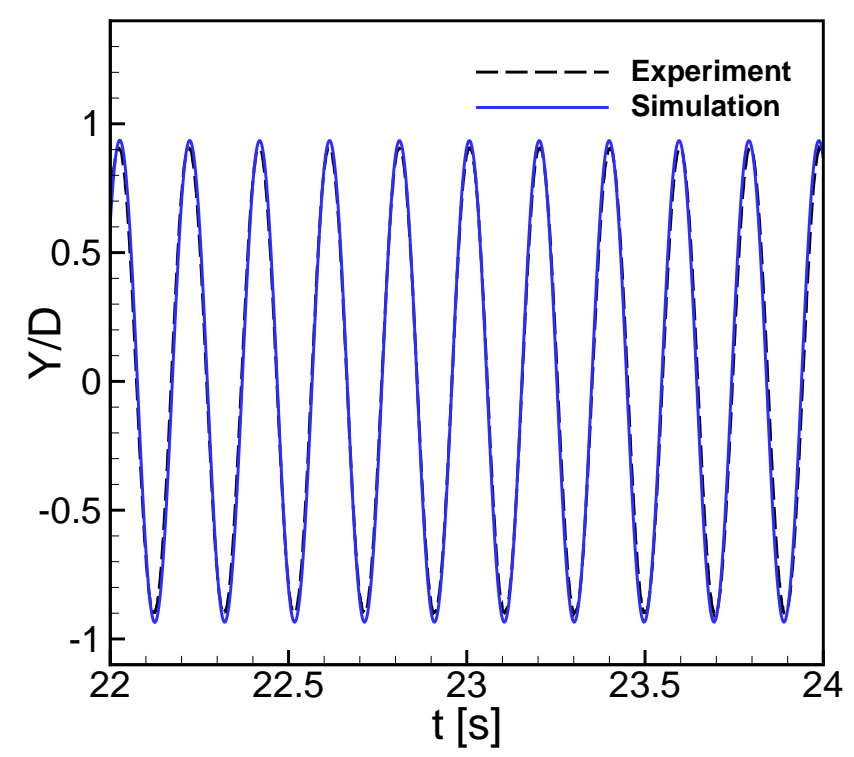

Figure B.27: Forced periodic oscillation test: Comparison of measured and computed ydisplacements of the point marked as measuring position in Fig. A.24 (Note that the experimental data are shifted by the offset $\phi_{\text {offset }}$ as mentioned in the text).

\begin{tabular}{|c|c|c|r|}
\hline & Mesh $($ Length $\times$ Width $)$ & $U_{\text {tip }}[\mathrm{m}]$ & Error \\
\hline \multirow{3}{*}{ Subset case } & $10 \times 10$ & $1.31466 \mathrm{e}-02$ & $0.6 \%$ \\
& $20 \times 20$ & $1.32076 \mathrm{e}-02$ & $0.1 \%$ \\
& $30 \times 30$ & $1.32248 \mathrm{e}-02$ & $0.01 \%$ \\
Full case & $40 \times 40$ & $1.32267 \mathrm{e}-02$ & Ref. \\
\hline & $20 \times 30$ & $1.31873 \mathrm{e}-02$ & $0.4 \%$ \\
& $30 \times 90$ & $1.32281 \mathrm{e}-02$ & $0.1 \%$ \\
& $40 \times 120$ & $1.32413 \mathrm{e}-02$ & $0.01 \%$ \\
& & $1.32426 \mathrm{e}-02$ & Ref. \\
\hline
\end{tabular}

Table C.6: Grid study for the structure with the CSD solver Carat++.

\section{References}

Adrian, R. J., 1991. Particle-imaging techniques for experimental fluid mechanics. Annual Review of Fluid Mechanics 23 (1), 261-304.

Balint, T. S., Lucey, A. D., 2005. Instability of a cantilevered flexible plate in viscous channel flow. Journal of Fluids and Structures 20 (7), 893-912.

Bischoff, M., 1999. Theorie und Numerik einer dreidimensionalen Schalenformulierung. Ph.D. thesis, Institut für Baustatik, Universität Stuttgart, Germany.

Bischoff, M., Ramm, E., 1997. Shear deformable shell elements for large strains and rotations. Int. Journal for Numerical Methods in Engineering 40 (23), 4427-4449.

Bischoff, M., Ramm, E., 2000. On the physical significance of higher-order kinematic and static variables in a three-dimensional shell formulation. Int. Journal of Solids and Structures 37 (46), 6933-6960. 
Bischoff, M., Wall, W. A., Bletzinger, K.-U., Ramm, E., 2004. Models and finite elements for thin-walled structures. In: Stein, E., De Borst, R., Hughes, T. J. R. (Eds.), Encyclopedia of Computational Mechanics. Vol. 2. John Wiley \& Sons Ltd, Chichester, pp. 59-138.

Bletzinger, K.-U., Wüchner, R., Daoud, F., Camprubí, N., 2005. Computational methods for form finding and optimization of shells and membranes. Computer Methods in Applied Mechanics and Engineering 194 (30), 3438-3452.

Bletzinger, K.-U., Wüchner, R., Kupzok, A., 2006. Algorithmic treatment of shells and free form-membranes in FSI. In: Bungartz, H.-J., Schäfer, M. (Eds.), Fluid-Structure Interaction. Vol. 53 of Lecture Notes in Computational Science and Engineering, LNCSE. Springer, Heidelberg, pp. 336-355.

Boyer, F., De Nayer, G., Leroyer, A., Visonneau, M., 2011. Geometrically exact Kirchhoff beam theory: Application to cable dynamics. Journal of Computational and Nonlinear Dynamics 6,041004 .

Breuer, M., 2002. Direkte Numerische Simulation und Large-Eddy Simulation turbulenter Strömungen auf Hochleistungsrechnern. Habilitationsschrift, Universität Erlangen-Nürnberg, Berichte aus der Strömungstechnik. Shaker Verlag, Aachen, Germany.

Breuer, M., De Nayer, G., Münsch, M., Gallinger, T., Wüchner, R., 2012. Fluid-structure interaction using a partitioned semi-implicit predictor-corrector coupling scheme for the application of large-eddy simulation. Journal of Fluids and Structures 29, 107-130.

Büchter, N., Ramm, E., 1992. Shell theory versus degeneration - A comparison in large rotation finite element analysis. Int. Journal for Numerical Methods in Engineering 34 (1), 39-59.

Büchter, N., Ramm, E., Roehl, D., 1994. Three-dimensional extension of non-linear shell formulation based on the enhanced assumed strain concept. Int. Journal for Numerical Methods in Engineering 37 (15), 2551-2568.

Bungartz, H.-J., Mehl, M., Schäfer, M. (Eds.), 2010. Fluid Structure Interaction II: Modelling, Simulation, Optimization. Vol. 73 of Lecture Notes in Computational Science and Engineering, LNCSE. Springer, Heidelberg.

Cantwell, B., Coles, D., 1983. An experimental study of entrainment and transport in the turbulent near wake of a circular cylinder. Journal of Fluid Mechanics 136 (1), 321-374.

Chung, J., Hulbert, G. M., 1993. A time integration algorithm for structural dynamics with improved numerical dissipation: The generalized- $\alpha$ method. Journal of Applied Mechanics $60,371-375$.

Clough, R. W., Penzien, J., 1993. Dynamics of Structures. McGraw-Hill, New York.

De Langre, E., 2002. Fluides et solides. Editions Ecole Polytechnique.

Demirdžić, I., Perić, M., 1988. Space conservation law in finite-volume calculations of fluid flow. Int. Journal for Numerical Methods in Fluids 8 (9), 1037-1050.

Demirdžić, I., Perić, M., 1990. Finite-volume method for prediction of fluid flow in arbitrarily shaped domains with moving boundaries. Int. Journal for Numerical Methods in Fluids 10 (7), 771-790. 
Dieringer, F., Wüchner, R., Bletzinger, K.-U., 2012. Practical advances in numerical form finding and cutting pattern generation for membrane structures. Journal of the International Association for Shell and Spatial Structures 53 (3), 147-156.

Durst, F., Schäfer, M., 1996. A parallel block-structured multigrid method for the prediction of incompressible flows. Int. Journal for Numerical Methods in Fluids 22 (6), 549-565.

Durst, F., Schäfer, M., Wechsler, K., 1996. Efficient simulation of incompressible viscous flows on parallel computers. In: Hirschel, E. H. (Ed.), Flow Simulation with High-Performance Computers II, Notes on Numerical Fluid Mechanics. Vol. 52(1). Vieweg, pp. 87-101.

Farhat, C., Lesoinne, M., LeTallec, P., 1998. Load and motion transfer algorithms for fluidstructure interaction problems with non-matching discrete interfaces: Momentum and energy conservation, optimal discretization and application to aeroelasticity. Computer Methods in Applied Mechanics and Engineering 157, 95-114.

Fischer, M., Firl, M., Masching, H., Bletzinger, K.-U., 2010. Optimization of non-linear structures based on object-oriented parallel programming. In: Topping, B. H. V., Adam, J. M., Pallarés, F. J., Bru, R., Romero, M. L. (Eds.), Seventh Int. Conf. Engineering Computational Technology, ECT2010. Civil-Comp Press, Stirlingshire, UK, p. 67.

Formaggia, L., Gerbeau, J. F., Nobile, F., Quarteroni, A., 2001. On the coupling of 3D and 1D Navier-Stokes equations for flow problems in compliant vessels. Computer Methods in Applied Mechanics and Engineering 191 (6-7), 561-582.

Förster, C., Wall, W. A., Ramm, E., 2007. Artificial added mass instabilities in sequential staggered coupling of non-linear structures and incompressible viscous flows. Computer Methods in Applied Mechanics and Engineering 196 (7), 1278-1293.

Gallinger, T., Kupzok, A., Israel, U., Bletzinger, K.-U., Wüchner, R., 2009. A computational environment for membrane-wind interaction. In: Hartmann, S., Mesiter, A., Schäfer, M., Turek, S. (Eds.), Int. Workshop on Fluid-Structure Interaction: Theory, Numerics and Applications. Kassel University Press GmbH, pp. 283-294.

Glück, M., Breuer, M., Durst, F., Halfmann, A., Rank, E., 2001. Computation of fluid-structure interaction on lightweight structures. Journal of Wind Engineering and Industrial Aerodynamics 89 (14-15), 1351-1368.

Gomes, J. P., 2011. Fluid-structure interaction-induced oscillation of flexible structures in uniform flows. Ph.D. thesis, Lehrstuhl für Strömungsmechanik, Universität Erlangen-Nürnberg, Germany.

Gomes, J. P., Lienhart, H., 2006. Experimental study on a fluid-structure interaction reference test case. In: Bungartz, H.-J., Schäfer, M. (Eds.), Fluid-Structure Interaction - Modelling, Simulation, Optimization. Vol. 53 of Lecture Notes in Computational Science and Engineering, LNCSE. Springer, Heidelberg, pp. 356-370.

Gomes, J. P., Lienhart, H., 2010. Experimental benchmark: Self-excited fluid-structure interaction test cases. In: Bungartz, H.-J., Mehl, M., Schäfer, M. (Eds.), Fluid-Structure Interaction II - Modelling, Simulation, Optimization. Vol. 73 of Lecture Notes in Computational Science and Engineering, LNCSE. Springer, Heidelberg, pp. 383-411. 
Gomes, J. P., Lienhart, H., 2013. Fluid-structure interaction-induced oscillation of flexible structures in laminar and turbulent flows. Journal of Fluid Mechanics 715, 537-572.

Gomes, J. P., Münsch, M., Breuer, M., Lienhart, H., 2010. Flow-induced oscillation of a flat plate - a fluid-structure interaction study using experiment and LES. In: Dillmann, A., Heller, G., Klaas, M., Kreplin, H., Nitsche, W., Schröder, W. (Eds.), New Results in Numerical and Experimental Fluid Mechanics VII, Contr. to the 16. STAB/DGLR Symposium, Nov. 3-5, 2008, Aachen, Germany. Vol. 112 of Notes on Numerical Fluid Mechanics and Multidisciplinary Design. Springer, Heidelberg, pp. 347-354.

Gomes, J. P., Yigit, S., Lienhart, H., Schäfer, M., 2011. Experimental and numerical study on a laminar fluid-structure interaction reference test case. Journal of Fluids and Structures 27 (1), 43-61.

Hojjat, M., Stavropoulou, E., Gallinger, T., Israel, U., Wüchner, R., Bletzinger, K.-U., 2010. Fluid-structure interaction in the context of shape optimization and computational wind engineering. In: Bungartz, H.-J., Mehl, M., Schäfer, M. (Eds.), Fluid-Structure Interaction II - Modelling, Simulation, Optimization. Vol. 73 of Lecture Notes in Computational Science and Engineering, LNCSE. Springer, Heidelberg, pp. 351-381.

Holzapfel, G. A., 2000. Nonlinear Solid Mechanics: A Continuum Approach for Engineering. John Wiley \& Sons Ltd.

Hübner, B., Walhorn, E., Dinkler, D., 2004. A monolithic approach to fluid-structure interaction using space-time finite elements. Computer Methods in Applied Mechanics and Engineering 193 (23-26), 2087-2104.

Hughes, T., Tezduyar, T., 1981. Finite elements based upon Mindlin plate theory with particular reference to the four-node bilinear isoparametric element. Journal of Applied Mechanics 48, 587-596.

Kalmbach, A., Breuer, M., 2013. Experimental PIV/V3V measurements of vortex-induced fluid-structure interaction in turbulent flow - a new benchmark FSI-PfS-2a. Journal of Fluids and Structures 42, 369-387.

Krätzig, W., Meskouris, K., Link, M., 1996. Der Ingenieurbau, Baustatik, Baudynamik. Ernst \& Sohn Verlag, Berlin, Ch. Baudynamik und Systemidentifikation, pp. 365-518.

Lesoinne, M., Farhat, C., 1996. Geometric conservation laws for flow problems with moving boundaries and deformable meshes, and their impact on aeroelastic computations. Computer Methods in Applied Mechanics and Engineering 134 (1-2), 71-90.

Mindlin, R., 1951. Influence of rotatory inertia and shear on flexural motions of isotropic, elastic plates. Journal of Applied Mechanics 18, 31-38.

Mok, D. P., 2001. Partitionierte Lösungsansätze in der Strukturdynamik und der FluidStruktur-Interaktion. Ph.D. thesis, Institut für Baustatik, Universität Stuttgart, Germany.

Naudascher, E., Rockwell, D., 1994. Flow-induced Vibrations: An Engineering Guide. AA Balkema, Rotterdam, Holland. 
Nobile, F., 2001. Numerical approximation of fluid-structure interaction problems with application to haemodynamics. Ph.D. thesis, Ecole Polytechnique Féderale de Lausanne, Switzerland.

Park, K. C., Stanley, G. M., 1986. A curved $C^{0}$-shell element based on assumed naturalcoordinate strains. Journal of Applied Mechanics 53, 278-290.

Petersen, C., 2000. Dynamik der Baukonstruktionen. Vieweg.

Reissner, E., 1945. The effect of transverse shear deformation on the bending of elastic plates. Journal of Applied Mechanics 12 (2), 69-77.

Reynolds, W. C., Hussain, A., 1972. The mechanics of an organized wave in turbulent shear flow. Part 3. Theoretical models and comparisons with experiments. Journal of Fluid Mechanics 54 (2), 263-288.

Smagorinsky, J., 1963. General circulation experiments with the primitive equations I: The basic experiment. Monthly Weather Review 91 (3), 99-165.

Taylor, R. L., 2002. FEAP-a Finite Element Analysis Program. Version 7.4 User Manual. University of California at Berkeley. http://www. ce.berkeley.edu/projects/feap/.

Thompson, J. F., Warsi, Z. U. A., Wayne Mastin, C. W., 1985. Numerical Grid Generation: Foundations and Applications. Vol. 45. North-Holland.

Turek, S., Hron, J., 2006. Proposal for numerical benchmarking of fluid-structure interaction between an elastic object and laminar incompressible flow. In: Bungartz, H.-J., Schäfer, M. (Eds.), Fluid-Structure Interaction. Vol. 53 of Lecture Notes in Computational Science and Engineering, LNCSE. Springer, Heidelberg, pp. 371-385.

Turek, S., Hron, J., Razzaq, M., Wobker, H., Schäfer, M., 2010. Numerical benchmarking of fluid-structure interaction: A comparison of different discretization and solution approaches. In: Bungartz, H.-J., Mehl, M., Schäfer, M. (Eds.), Fluid-Structure Interaction II - Modelling, Simulation, Optimization. Vol. 73 of Lecture Notes in Computational Science and Engineering, LNCSE. Springer, Heidelberg, pp. 413-424.

Wall, W. A., 1999. Fluid-Struktur-Interaktion mit stabilisierten Finiten Elementen. Ph.D. thesis, Institut für Baustatik, Universität Stuttgart, Germany.

Wall, W. A., Ramm, E., 1998. Fluid-structure interaction based upon a stabilized (ALE) finite element method. IV World Congress on Computational Mechanics, Barcelona.

Wüchner, R., Bletzinger, K.-U., 2005. Stress-adapted numerical form finding of pre-stressed surfaces by the updated reference strategy. Int. Journal for Numerical Methods in Engineering $64(2), 143-166$.

Wüchner, R., Kupzok, A., Bletzinger, K.-U., 2007. A framework for stabilized partitioned analysis of thin membrane-wind interaction. Int. Journal for Numerical Methods in Fluids 54 (6-8), 945-963. 